\title{
Improved Jamming-Resistant Frequency Hopping SpRead SPECTRUM SYSTEMS
}

\author{
by
}

Walid Mohamed Saad Aly Atta

\begin{abstract}
A THEsis SUBMitTed TO THE
Faculty of Graduate and Postdoctoral Affairs

IN PARTIAL FULFILLMENT OF THE REQUIREMENTS FOR THE DEGREE OF

Doctor of Philosophy
\end{abstract}

Ottawa-Carleton Institute for Electrical and Computer Engineering Department of Systems and Computer Engineering

Carleton University

Ottawa, Ontario

January 2014

(c) Walid Mohamed Saad Aly Atta, 2014 
The undersigned recommend to

the Faculty of Graduate Studies and Postdoctoral Affairs

acceptance of the thesis

\title{
Improved Jamming-Resistant Frequency Hopping Spread Spectrum Systems
}

\author{
submitted by \\ Walid Mohamed Saad Aly Atta
}

in partial fulfillment of the requirements for the degree of

Doctor of Philosophy

Chair, Professor Roshdy Hafez, Department of Systems and Computer Engineering

Professor Ian D. Marsland, Thesis Supervisor

External Examiner, Professor Walaa Hamouda, Faculty of Engineering, Concordia University

Carleton University

January 2014 


\begin{abstract}
Frequency hopping spread spectrum is widely used in military applications to provide communication between command posts, soldiers, vehicles, sensors, missile launchers, etc. It provides good protection against the effects of frequency selective fading, and can be robust in jamming environments. Traditional frequency hopping spread spectrum involves dividing the available spectrum into a large number of sub-bands, and hopping over these sub-bands in a pseudo-random fashion, but there are different implementations within this general framework that can deliver better performance.

In this thesis, we present several existing frequency hopping spread spectrum schemes and we investigate their performance in the presence of frequency selective fading and adaptive partial band jamming. We optimize the control parameters of matched frequency hopping, clipped matched frequency hopping and advanced frequency hopping to enhance their throughput performance. We also propose three new random frequency hopping schemes that generate a hopping pattern with the property of being random over the total bandwidth of the channel but good sub-bands tend to be selected more frequently. These new random frequency hopping schemes provide greater resilience to adaptive jamming and give much higher throughput than the existing schemes.
\end{abstract}




\section{Acknowledgements}

First and foremost, I am grateful to Allah (God) for all that I am and all that I have. I am very thankful for the patient guidance and the endless support and encouragement given by my supervisor Dr. Ian Marsland. I am also thankful to the defence committee members, Dr. Walaa Hamouda, Dr. Yongyi Mao, Dr. Halim Yanikomeroglu, and Dr. John Rogers, for their valuable comments and discussions which greatly improved the content of this thesis.

I would like to express my gratefulness to my local supervisor in Egypt Dr. Ali Gaballa for his continuous support until I did my defense. His assistance can never be appreciated enough.

I would like to express my gratitude to Dr. Ahmed Ahmed and Dr. Mostafa Mohammed for their continuous support since my first day in Ottawa.

Many thanks go to my colleagues, Dr. Ramy Gohary, Mohamed Mabrouk, Wael moussa, Dr. Tarik Shehata, Dr. Akram Bin Sediq, Dr. Mohamed Rashad, Dr. Rajab Legnain, Tamer Beitelmal, Hossein Chahrour, and Suzan Ureten for their encouragement.

To my parents, parents in law, and sister, I must express my sincere appreciation for their guidance, support, and love in every aspect of my life. Finally, I thank my lovely wife Mai for her patience, encouragement, support, and love. 


\section{Table of Contents}

Abstract $\quad$ ii

Acknowledgements

Table of Contents $\quad$ iv

List of Tables $\quad$ vi

List of Figures $\quad$ vii

List of Acronyms $\quad$ xi

List of Symbols $\quad$ xiii

Chapter 1: Introduction $\quad 1$

1.1 Overview of Spread Spectrum Techniques . . . . . . . . . . . . . . 3

1.2 Jamming Strategies . . . . . . . . . . . . . . . . . . . 6

1.2.1 Barrage Noise Jammer . . . . . . . . . . . . . . . . 6

1.2.2 Partial Band Jammer . . . . . . . . . . . . . . . . . . . . . 6

1.2.3 Single Tone Jammer . . . . . . . . . . . . . . . . . . . . . . 7

1.2.4 Multiple Tone Jammer . . . . . . . . . . . . . . . . . . . . . . . 7

1.2 .5 ON-OFF Jammer . . . . . . . . . . . . . . . . 8

1.2.6 Repeater Jammer . . . . . . . . . . . . . . . . . . 8

1.2 .7 Smart Jammer . . . . . . . . . . . . . . . . . . . 8

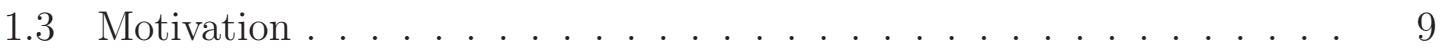

1.4 Literature Review . . . . . . . . . . . . . . . . . . . . . . . 10

1.5 Contributions . . . . . . . . . . . . . . . . 15

1.6 Thesis Outline . . . . . . . . . . . . . . . 16

Chapter 2: $\quad$ Review of Frequency Hopping Spread Spectrum 18

2.1 Frequency Hopping Spread Spectrum . . . . . . . . . . . . . . . 18

2.2 Hopping Set Selection Algorithms . . . . . . . . . . . . . . . . . 22

2.2.1 Highest Gain Frequency Hopping . . . . . . . . . . . . . . . . 22

2.2.2 Matched Frequency Hopping . . . . . . . . . . . . . . . . 23

2.2.3 Clipped Matched Frequency Hopping . . . . . . . . . . . . . . 26

2.2.4 Advanced Frequency Hopping . . . . . . . . . . . . . . . 26

2.3 System Model . . . . . . . . . . . . . . . . . . . . . . . . 27 
Chapter 3: Optimization of Frequency Hopping Schemes 34

3.1 Simulation Environment and Performance Metric . . . . . . . . . . 34

3.2 Performance of the Original Schemes . . . . . . . . . . . . . . . 35

3.3 Parametric Optimization . . . . . . . . . . . . . . . . . 38

3.3.1 Optimized Matched Frequency Hopping (OMFH) . . . . . . . 39

3.3.2 Optimized Clipped Matched Frequency Hopping (OCMFH) . 43

3.3.3 Optimized Advanced Frequency Hopping (OAFH) . . . . . . . 51

3.4 Comparisons . . . . . . . . . . . . . . . . . . . . . 58

3.5 Performance Comparison in Different Channel Models . . . . . . . . . 62

3.6 The Effect of Different Jamming Bandwidth . . . . . . . . . . . . 65

3.7 Conclusions $\ldots \ldots \ldots \ldots \ldots$

Chapter 4: Weighted Random Frequency Hopping $\quad 70$

4.1 Weighted Random Frequency Hopping . . . . . . . . . . . . . . 70

4.2 Advanced Weighted Random Frequency Hopping . . . . . . . . . . 77

4.3 Clipped Weighted Random Frequency Hopping . . . . . . . . . . . . 83

4.4 Performance Comparison . . . . . . . . . . . . . . . . . . . . . 87

4.5 Conclusion . . . . . . . . . . . . . . . . . . . . . . . . 89

$\begin{array}{lll}\text { Chapter 5: } & \text { Conclusions and Future Work } & 91\end{array}$

5.1 Summary and Conclusions . . . . . . . . . . . . . . . . . . . . 91

5.2 Suggestions for Future Work . . . . . . . . . . . . . . . . . 92

$\begin{array}{ll}\text { References } & 94\end{array}$

Appendix A: Throughput in Wide Band Jamming 102 


\section{List of Tables}

3.1 WINNER D1 channel model parameters . . . . . . . . . . . . . 


\section{List of Figures}

1.1 Two-way communication in the presence of jamming in frequency selective fading channel. . . . . . . . . . . . . . . . 99 9

2.1 Frequency hopping patterns. . . . . . . . . . . . . . . . 19

2.2 Block diagram of a frequency hopping system. . . . . . . . . . . . 20

2.3 Example of a HGFH hopping set where $M=15$ sub-bands are selected out of $K=100$. Vertical black lines indicate selected sub-bands. . . . 23

2.4 Illustration of selecting $M=15$ sub-bands out of $K=100$ using MFH in a simple channel. . . . . . . . . . . . . . . . . . . 25

2.5 MFH hopping set of $M=15$ sub-bands for a sample channel realization. 25

2.6 CMFH hopping set with $M=15$ sub-bands. . . . . . . . . . . . 27

2.7 AFH hopping set of $M=15$ sub-bands. . . . . . . . . . . . . . . . . . 28

2.8 System model flowchart. . . . . . . . . . . . . . . . . . . . . . . . . 29

2.9 The modulation-constrained channel capacity of noncoherently detected binary FSK over the AWGN channel. . . . . . . . . . . . . . . 31

3.1 Average throughput performance of the frequency hopping schemes in the absence of jamming. . . . . . . . . . . . . . . . . 36

3.2 Illustrative example of the hopping sets selected by the different schemes. 37

3.3 Average throughput of the hopping schemes in the presence of adaptive jamming. . . . . . . . . . . . . . . . . . 38

3.4 The throughput vs. the size of the hopping set for $\mathrm{MFH}$ with different jamming powers and 5 dB SNR. . . . . . . . . . . . . 39

3.5 The throughput vs. the size of the hopping set for $\mathrm{MFH}$ with different jamming powers and $0 \mathrm{~dB}$ SNR. . . . . . . . . . .

3.6 The throughput vs. the size of the hopping set for $\mathrm{MFH}$ with different jamming powers and $10 \mathrm{~dB}$ SNR. . . . . . . . . . . . . 41

3.7 The optimum hopping set size for $\mathrm{MFH}$ with the presence of different jamming power and SNR scenarios. . . . . . . . . . . . . . . 42

3.8 The optimum throughput of MFH for different jamming power and SNR scenarios. . . . . . . . . . . . . . . . . . . . . 43

3.9 Throughput comparison of $\mathrm{MFH}$ and $\mathrm{OMFH}$ when the $\mathrm{SNR}=0 \mathrm{~dB} . \quad 44$

3.10 Throughput comparison of $\mathrm{MFH}$ and $\mathrm{OMFH}$ when the $\mathrm{SNR}=5 \mathrm{~dB} . \quad 44$

3.11 Throughput comparison of $\mathrm{MFH}$ and $\mathrm{OMFH}$ when the $\mathrm{SNR}=10 \mathrm{~dB} . \quad 45$

3.12 The throughput performance of CMFH vs. $T$ when $M=15$ and SNR $=5 \mathrm{~dB}$ for different jamming power scenarios. . . . . . . . . . . 46 
3.13 The throughput performance of $\mathrm{CMFH}$ vs. $M$ when $T=0.2$ and $\mathrm{SNR}$ $=5 \mathrm{~dB}$ for different jamming power scenarios. . . . . . . . . . .

3.14 The throughput vs. the size of the hopping set for $\mathrm{CMFH}$ with different jamming powers and $5 \mathrm{~dB}$ SNR. The optimum threshold is used for each $M \ldots \ldots \ldots \ldots \ldots \ldots \ldots \ldots$

3.15 The requested number of sub-bands vs. the actual number of used sub-bands. . . . . . . . . . . . . . . . .

3.16 The throughput vs. the threshold value for $\mathrm{CMFH}$ with different jamming powers and $5 \mathrm{~dB}$ SNR. The optimal hopping set size is used for each $T \ldots \ldots \ldots \ldots \ldots \ldots \ldots \ldots$

3.17 The optimum threshold for CMFH with the presence of different jamming power and SNR scenarios. . . . . . . . . . . . .

3.18 The optimum number of sub-bands for $\mathrm{CMFH}$ with the presence of different jamming power and SNR scenarios. . . . . . . . . . . . . 50

3.19 The optimum throughput for $\mathrm{CMFH}$ with the presence of different jamming power and SNR scenarios. . . . . . . . . . . . 51

3.20 Throughput comparison of CMFH and OCMFH when the $\mathrm{SNR}=0 \mathrm{~dB} .52$

3.21 Throughput comparison of $\mathrm{CMFH}$ and $\mathrm{OCMFH}$ when the $\mathrm{SNR}=5 \mathrm{~dB} .52$

3.22 Throughput comparison of $\mathrm{CMFH}$ and $\mathrm{OCMFH}$ when the $\mathrm{SNR}=10$

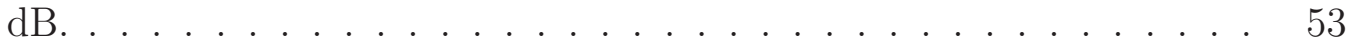

3.23 The throughput vs. the size of the hopping set for AFH with different jamming powers and $5 \mathrm{~dB}$ SNR. . . . . . . . . . . . .

3.24 The throughput vs. the regulation value for $\mathrm{AFH}$ with different jamming powers and 5 dB SNR. . . . . . . . . . . . . . 54

3.25 The optimum throughput for AFH with the presence of different jamming power and SNR scenarios. . . . . . . . . . . .

3.26 The optimum number of sub-bands for $\mathrm{OAFH}$ with the presence of different jamming power and SNR scenarios. . . . . . . . . . . .

3.27 The optimum threshold for OAFH with the presence of different jamming power and SNR scenarios. . . . . . . . . . . . 56

3.28 Throughput comparison of $\mathrm{AFH}$ and $\mathrm{OAFH}$ when the $\mathrm{SNR}=0 \mathrm{~dB} . .57$

3.29 Throughput comparison of $\mathrm{AFH}$ and $\mathrm{OAFH}$ when the $\mathrm{SNR}=5 \mathrm{~dB} . \quad .57$

3.30 Throughput comparison of AFH and OAFH when the SNR $=10 \mathrm{~dB} . \quad 58$

3.31 Throughput comparison of OMFH, OCMFH and OAFH when the $\mathrm{SNR}=5 \mathrm{~dB} \ldots \ldots \ldots \ldots \ldots$

3.32 Throughput comparison of $\mathrm{OMFH}, \mathrm{OCMFH}$ and $\mathrm{OAFH}$ when the $\mathrm{SNR}=-5 \mathrm{~dB} \ldots \ldots \ldots \ldots$. . . . . . . . . . . . 60

3.33 Throughput comparison of OMFH, OCMFH and OAFH when the

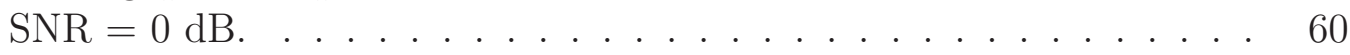

3.34 Throughput comparison of $\mathrm{OMFH}, \mathrm{OCMFH}$ and $\mathrm{OAFH}$ when the $\mathrm{SNR}=10 \mathrm{~dB} \ldots \ldots \ldots \ldots$

3.35 Throughput comparison of OMFH, OCMFH and OAFH when the

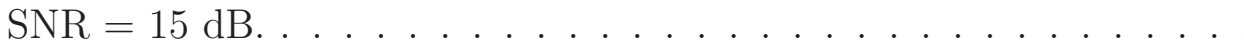


3.36 Throughput comparison of OMFH, OCMFH and OAFH when the $\mathrm{SNR}=-5 \mathrm{~dB}$ in the presence of D1-LOS WINNER channel model.

3.37 Throughput comparison of OMFH, OCMFH and OAFH when the $\mathrm{SNR}=0 \mathrm{~dB}$ in the presence of D1-LOS WINNER channel model. . .

3.38 Throughput comparison of $\mathrm{OMFH}, \mathrm{OCMFH}$ and $\mathrm{OAFH}$ when the $\mathrm{SNR}=5 \mathrm{~dB}$ in the presence of D1-LOS WINNER channel model. . .

3.39 Throughput comparison of OMFH, OCMFH and OAFH when the $\mathrm{SNR}=10 \mathrm{~dB}$ in the presence of D1-LOS WINNER channel model.

3.40 Throughput comparison of OMFH, OCMFH and OAFH when the $\mathrm{SNR}=15 \mathrm{~dB}$ in the presence of D1-LOS WINNER channel model.

3.41 Throughput comparison of OMFH, OCMFH and OAFH when the $\mathrm{SNR}=-5 \mathrm{~dB}$ in the presence of $\mathrm{C} 2-\mathrm{NLOS}$ WINNER channel model. .

3.42 Throughput comparison of OMFH, OCMFH and OAFH when the $\mathrm{SNR}=0 \mathrm{~dB}$ in the presence of $\mathrm{C} 2-\mathrm{NLOS}$ WINNER channel model. .

3.43 Throughput comparison of OMFH, OCMFH and OAFH when the $\mathrm{SNR}=5 \mathrm{~dB}$ in the presence of C2-NLOS WINNER channel model. .

3.44 Throughput comparison of OMFH, OCMFH and $\mathrm{OAFH}$ when the $\mathrm{SNR}=10 \mathrm{~dB}$ in the presence of C2-NLOS WINNER channel model.

3.45 Throughput comparison of OMFH, OCMFH and OAFH when the $\mathrm{SNR}=15 \mathrm{~dB}$ in the presence of C2-NLOS WINNER channel model.

3.46 The optimum throughput for OCMFH with the presence of different jamming bandwidth and SNR scenarios. . . . . . . . . . .

4.1 The performance of WRFH in different jamming power and SNR scenarios. . . . . . . . . . . . . . . . . . .

4.2 The performance of WRFH compared to OMFH and RFH in the absence of jamming. . . . . . . . . . . . . . . .

4.3 The performance of WRFH compared to OMFH and RFH when SJR

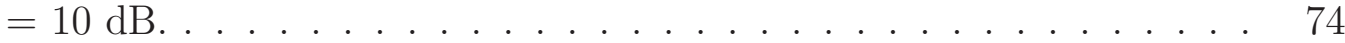

4.4 The performance of WRFH compared to OMFH and RFH when SJR

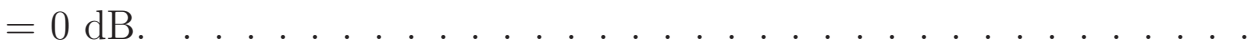

4.5 The performance of WRFH compared to OMFH and RFH with the presence of infinite jamming. . . . . . . . . . . . . . . . . . . 75

4.6 The throughput vs. the regulation value for AWRFH with different jamming powers and 0 dB SNR. . . . . . . . . . . . . . . . . . . . 78

4.7 The throughput vs. the regulation value for AWRFH with different jamming powers and $5 \mathrm{~dB}$ SNR. . . . . . . . . . . . .

4.8 The throughput vs. the regulation value for AWRFH with different jamming powers and $10 \mathrm{~dB}$ SNR. . . . . . . . . . . . . . . . . . . 79

4.9 The optimum regulation value for $\mathrm{AWRFH}$ in different jamming power and SNR scenarios. . . . . . . . . . . . . . . . . 79

4.10 The optimum throughput for AWRFH in different jamming power and SNR scenarios. 
4.11 The performance of AWRFH compared to OAFH and WRFH in the absence of jamming. . . . . . . . . . . . . . . . . . . . . . . 81

4.12 The performance of AWRFH compared to OAFH and WRFH when $\mathrm{SJR}=10 \mathrm{~dB} \ldots \ldots \ldots \ldots$. . . . . . . . . . . . 81

4.13 The performance of AWRFH compared to OAFH and WRFH when $\mathrm{SJR}=0 \mathrm{~dB} \ldots \ldots \ldots \ldots$

4.14 The performance of AWRFH compared to OAFH and WRFH in the presence of infinite jamming. . . . . . . . . . . . . . . . 82

4.15 The optimum threshold for CWRFH in different jamming power and SNR scenarios. . . . . . . . . . . . . . . . . 83

4.16 The optimum throughput for CWRFH in different jamming power and SNR scenarios. . . . . . . . . . . . . . . . . 84

4.17 The performance of OCWRFH compared to OCMFH and WRFH with absence of jamming. . . . . . . . . . . . . . . . . . 85

4.18 The performance of CWRFH compared to OCMFH and WRFH when $\mathrm{SJR}=10 \mathrm{~dB} \ldots \ldots \ldots \ldots$

4.19 The performance of CWRFH compared to OCMFH and WRFH when $\mathrm{SJR}=0 \mathrm{~dB} \ldots \ldots \ldots \ldots$. . . . . . . . . . . 86

4.20 The performance of OCWRFH compared to OCMFH and WRFH with the presence of infinite jamming. . . . . . . . . . . . . . 86

4.21 The comparison of all proposed schemes vs. SJR when $\mathrm{SNR}=-5 \mathrm{~dB} . \quad 87$

4.22 The comparison of all proposed schemes vs. SJR when SNR $=0 \mathrm{~dB} . .88$

4.23 The comparison of all proposed schemes vs. SJR when $\mathrm{SNR}=5 \mathrm{~dB} . . \quad 88$

4.24 The comparison of all proposed schemes vs. SJR when SNR $=10 \mathrm{~dB} . \quad 89$ 


\section{List of Acronyms}

$\begin{array}{ll}\text { ACI } & \text { Adjacent Channel Interference } \\ \text { AFH } & \text { Advanced Frequency Hopping } \\ \text { AM } & \text { Amplitude Modulation } \\ \text { ARQ } & \text { Automatic Repeat reQuest } \\ \text { ASK } & \text { Amplitude Shift Keying } \\ \text { AWGN } & \text { Additive White Gaussian Noise } \\ \text { AWRFH } & \text { Advanced Weighted Random Frequency Hopping } \\ \text { BER } & \text { Bit Error Rate } \\ \text { BPSK } & \text { Binary Phase Shift Keying } \\ \text { CAFHSS } & \text { Channel Aware Frequency Hopping Spread Spectrum } \\ \text { CCI } & \text { Co-Channel Interference } \\ \text { CDMA } & \text { Code Division Multiple Access } \\ \text { CIFHSS } & \text { Channel Ignorant Frequency Hopping Spread Spectrum } \\ \text { CMFH } & \text { Clipped Matched Frequency Hopping } \\ \text { CPFSK } & \text { Continuous Phase Frequency Shift Keying } \\ \text { CWRFH } & \text { Clipped Weighted Random Frequency Hopping } \\ \text { DAFH } & \text { Dynamic Adaptive Frequency Hopping } \\ \text { dB } & \text { Decibel } \\ \text { DFH } & \text { Dynamic Frequency Hopping } \\ \text { DPSK } & \text { Differential Phase Shift Keying } \\ \text { DS } & \text { Direct Sequence } \\ \text { DSSS } & \text { Direct Sequence Spread Spectrum } \\ \text { FDMA } & \text { Frequency Division Multiple Access } \\ \text { FFH/BFSK } & \text { Fast Frequency Hopping Binary Frequency Shift Keying } \\ \text { FH/MFSK } & \text { Frequency Hopping M'ary Frequency Shift Keying } \\ \text { FHSS } & \text { Frequency Hopping Spread Spectrum } \\ \text { FM } & \text { Frequency Modulation } \\ \text { FSK } & \text { Frequency Shift Keying } \\ \text { GPS } & \text { Global Positioning Systems } \\ \text { HGFH } & \text { High Gain Frequency Hopping } \\ \text { IF } & \text { Intermediate Frequency } \\ \text { IFH } & \text { Interleaved Frequency Hopping } \\ \text { ISI } & \text { Intersymbol Interference } \\ \text { MAI } & \text { Multiple Access Interference } \\ \text { MDFH } & \text { Message Driven Frequency Hopping } \\ \text { MFH } & \text { Matched Frequency Hopping } \\ \text { MFSK } & \text { M'ary Frequency Shift Keying } \\ & \end{array}$


MIMO Multiple Input Multiple Output

ML Maximum Likelihood

MPSK M'ary Phase Shift Keying

MTJ Multi-tone Jamming

OAFH Optimized Advanced Frequency Hopping

OCMFH Optimized Clipped Matched Frequency Hopping

OFDM Orthogonal Frequency Division Multiplexing

OFDMA Orthogonal Frequency Division Multiple Access

OMFH Optimized Matched Frequency Hopping

PAM Pulse Amplitude Modulation

PBJ Partial Band Jamming

PCM Pulse Code Modulation

PER Packet Error Rate

PSD Power Spectral Density

PSK Phase Shift Keying

RF Radio Frequency

RFH Random Frequency Hopping

SFH Slow Frequency Hopping

SIR Signal to Interference Ratio

SJR Signal to Jamming Ratio

SJNR Signal to Jamming and Noise Ratio

SNR Signal to Noise Ratio

WLAN Wireless Local Area Networks

WRFH Weighted Random Frequency Hopping 


\section{List of Symbols}

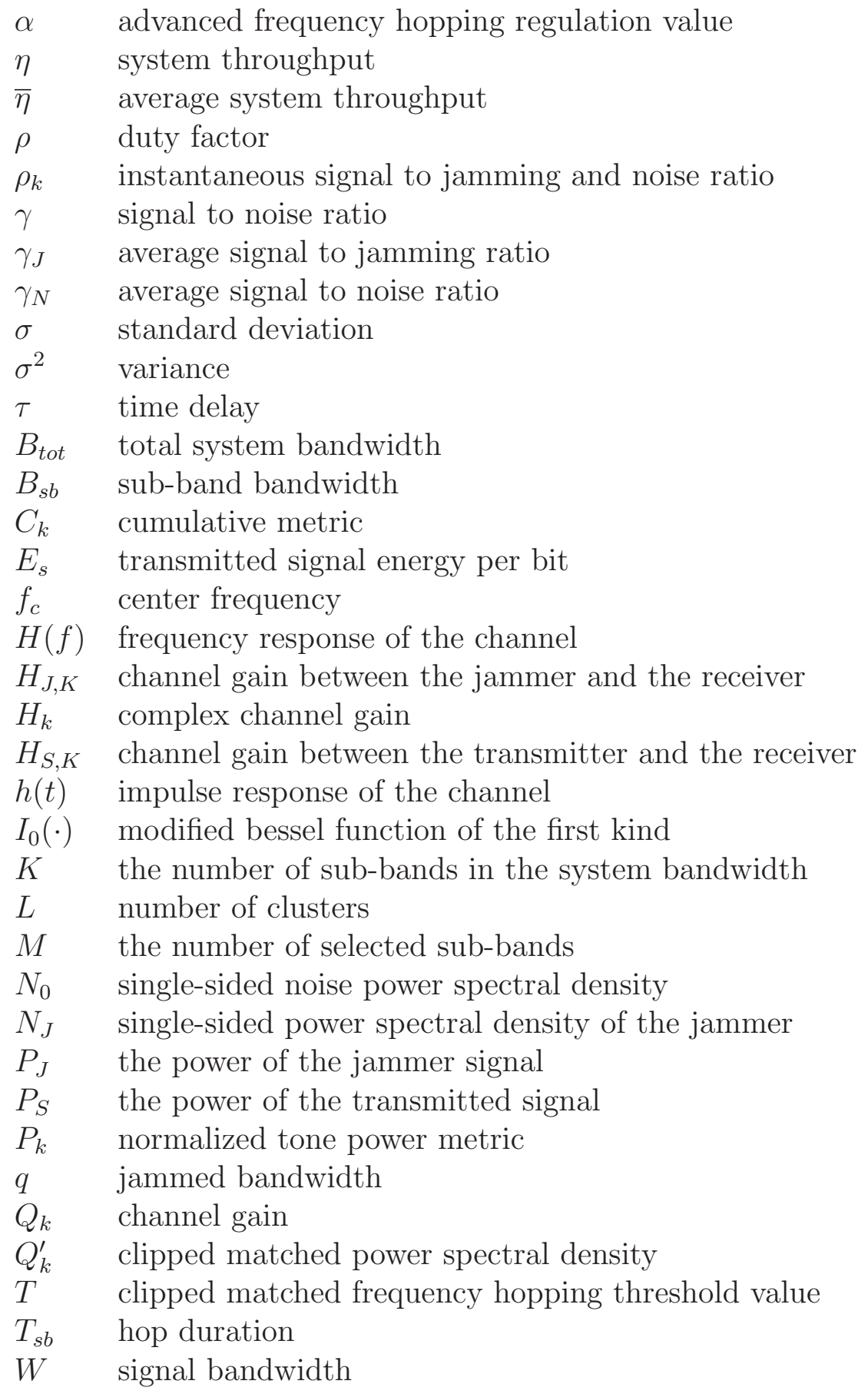




\section{Chapter 1}

\section{Introduction}

Reliable real-time communication is essential to the success of military operations. For example, communication is needed between posts, soldiers, missile lunchers, aircrafts and remote sensors. Because of the highly transitional nature of the theater of operation, wireline communication is often not feasible, so wireless communication systems must be deployed instead. Furthermore, the deployment must often be done hastily, without the luxury of careful coverage planning and analysis available when installing civilian cellular networks. As a result, military wireless communication systems present a challenging communication environment, needing to deal with impairments such as channel fading, interference and jamming, while also addressing security issues.

Channel fading is used to describe the rapid fluctuation of the amplitude of a radio signal over a short period of time or travel distance. Channel fading occurs because of the reception of multiple versions of a transmitted signal at the receiver due to reflections from the ground and surrounding structures, i.e., multipath signal propagation. The incoming radio waves arrive from different directions with different propagation delays. The received signal may consist of a large number of plane waves having randomly distributed amplitudes, phases, and angles of arrival. These multipath components combine vectorially at the receive antenna, and sometimes 
the relative phase shifts align so that the different signals add constructively, while at other times they cancel one another resulting in a weak received signal. The overall effect causes the received signal to distort or fade over time. Even when a receiver is stationary, the received signal may fade due to movement of surrounding objects in the radio channel. Spread spectrum modulation [1], [2], [3], diversity combining [4], [5], multiple-input multiple-output (MIMO) channels [6], and smart antennas [7] are some solutions for fading.

There are two types of fading: frequency flat fading, and frequency selective fading. In flat fading the bandwidth of the signal is smaller than the coherence bandwidth, which is a statistical measure of the range of frequencies over which the frequency response of the multipath channel can be considered flat, and in frequency selective fading the bandwidth of the signal is greater than the coherence bandwidth. In a frequency selective fading channel, the delay spread of the incoming waves is too long, and intersymbol interference (ISI) occurs. The presence of ISI makes it more difficult to recover the desired signal. Interference can also come from external sources, such as the environment or other users, either on the same or a different communication system. Examples include co-channel interference (CCI), a type of interference that results from reusing the same set of frequencies within a given coverage area, and adjacent channel interference (ACI), a type of interference that results from signals that are adjacent in frequency to the desired signal. Spread spectrum modulation [8], channel coding [9], equalization [10], rake and pre-rake techniques [11], [12], and orthogonal frequency division multiplexing (OFDM) [13] are solutions for channel interference.

Unlike interference, which is unintended, jamming is an intended effect caused by a hostile communicator or intentional interferer who is called a jammer. The jammer 
tries to disrupt the transmission of the signal by sending an interfering signal in the same communication frequency band. There are many jamming strategies such as barrage jamming, single tone jamming, partial band jamming and, multi-tone jamming. Spread spectrum modulation is found to be robust to mitigate different jamming strategies [14], [15], [16], [17], [18], [19].

Security needs for wireless systems became very important in both commercial and military applications, because more and more users are relying on wireless networks to transmit critical and private information. This information is vulnerable to be attacked or received by unwanted listeners. For that reason engineers started to look for solutions for wireless security problem. Some of the security solutions are spread spectrum techniques [20], watermarking [21], encryption [22], and secure modulation schemes [23].

Spread spectrum techniques are found to be a solution for many wireless communication challenges. It is a solution for channel fading, interference and jamming, and it can be used with secure random sequences to provide secure transmission.

\subsection{Overview of Spread Spectrum Techniques}

Spread spectrum techniques have many applications, such as jam-resistant communication systems, global positioning systems (GPS), wireless local area networks (WLAN), cordless phones, long range wireless phones for home and industry, cellular base station interconnection, and code division multiple access (CDMA) radio, which allows multiple users to access and use the communication system simultaneously.

Spread spectrum is a means of transmission where the signal uses a wide range of frequencies to send the information. The signal spreading is accomplished by different means of code generation at the transmitter and this signal spreading is independent 
of the data. The spread signals would be collected and then converted to their original frequency at the receiver after synchronizing the generated code with the code at the receiver. By spreading the transmitted signals they become more transparent, so it is unlikely that they will be intercepted by an opponent, or interfered with by other signals intended for other users, even if they use the same frequencies.

The concept of spread spectrum was first developed to ensure secure communications in military applications and the first approaches were undertaken more than sixty years ago [24]. Wideband modulation such as frequency modulation (FM) and pulse code modulation (PCM) schemes have a signal-to-noise-ratio (SNR) advantage over amplitude modulation (AM) and pulse amplitude modulation (PAM) [25], [26]. The first intentional use of spread spectrum, based upon wideband FM radio is attributed to E. Armstrong [27]. During World War II, FM was also used to mitigate the effects of jamming signals that were intended to disrupt AM communication channels. After H. Lamarr developed the frequency hopping spread spectrum (FHSS) technique in 1941 [28], more elaborate spread spectrum techniques were developed to reduce the likelihood of prediction of the bandwidth spreading process. Within a decade, M. Nicholson developed the first binary phase shift keying (BPSK) direct sequence spread spectrum (DSSS) communication system, in 1951 [27]. In the 1980's spread spectrum techniques were first used for commercial purposes when DSSS was used for multiple access over a satellite communication channel [29]. Then the CDMA technique was developed and became more common (e.g. Interim Standard-95). This technique is useful for improving frequency reuse for cellular communications [30], and also being used as overlays to existing narrowband (i.e. frequency division multiple access (FDMA)) communications systems [31]. The uses of spread spectrum have been extended to guidance systems, experimental anti-multipath systems, and 
many other applications.

Although spread spectrum was developed primarily to secure military communications, it was later used for commercial developments such as mobile radio networks (radio telephony, packet radio, and amateur radio), timing and positioning systems, specialized applications in satellites, etc.

There are many reasons for spreading the spectrum, and if it is done properly, many benefits can accrue simultaneously. Some of these benefits are:

1. Interference rejection,

2. Anti-jamming,

3. Low probability of intercept,

4. Multiple access,

5. High resolution ranging,

6. Multi-path suppression, and

7. Diversity reception.

There are several methods used to implement the spread spectrum technique. Each has its own design and features. The main spread spectrum methods are:

1. Direct sequence,

2. Frequency hopping,

3. Time hopping, and 
4. Hybrid (a combination of direct sequence and frequency hopping).

Each of these methods effectively provide a further level of modulation not directly associated with the information or sinusoidal carrier signal, thereby providing some immunity against interfering signals without a significant loss of information, or alternatively, reducing the likelihood of signal detection or interception. This is particulary important when intentional signal jamming is present in a hostile communication environment.

\section{$1.2 \quad$ Jamming Strategies}

Jamming is the ability to interfere, distort, or prevent the signal transmission before it received by its desired receiver. There are different ways to place the jamming signal within the spread spectrum bandwidth. In this section, the most effective and commonly used strategies of jamming will be presented.

\subsubsection{Barrage Noise Jammer}

The jammer transmits bandlimited white Gaussian noise. It is usually assumed that the jammer power spectrum covers exactly the same frequency range as the spread spectrum signal. The effect of the barrage noise jammer on the spectrum is to increase the Gaussian noise level at the output of the receiver down converter. If the power of the jammer signal is $P_{J}$ watts, and signal has a bandwidth of $W \mathrm{~Hz}$, the single-sided power spectral density (PSD) of the jammer is $N_{J}=P_{J} / W$.

\subsubsection{Partial Band Jammer}

To jam a spread spectrum signal, it is typically more effective to transmit all the available jamming power in a limited bandwidth. This is called a partial band 
jammer. If the fraction of the spread spectrum signal bandwidth which is jammed is denoted by $q$, the PSD of the partial band jammer is $N_{J}=P_{J} / q W$, where $P_{J}$ is the total jamming power and, $q W$ is the limited bandwidth of the signal which is jammed. The partial band jammer is particularly effective against frequency hopping spread spectrum systems because the signal will hop in and out of the jamming band and can be seriously degraded in the jamming band [32], [33].

\subsubsection{Single Tone Jammer}

The single tone jammer transmits an unmodulated carrier with power $P_{J}$ somewhere in the spread spectrum signal bandwidth. The single tone jammer is easily to generate and is rather effective against direct sequence spread spectrum systems. To achieve the maximum effectiveness of this jammer, the jamming tone should be placed at the center of the spread spectrum signal bandwidth. The single tone jammer is less effective against frequency hopping, since the frequency hopping instantaneous bandwidth is small and, for large processing gains the probability of any hop being jammed is small [33].

\subsubsection{Multiple Tone Jammer}

A better tone jamming strategy against frequency hopping systems is to use several tones instead of a single tone. However, the power of the single tone jammer will be shared by these multiple jamming tones. The jammer selects a number of tones so that the optimum degradation occurs when the spread spectrum signal hops to a jamming tone frequency. The optimum number of tones is a function of the received ratio of signal power to jammer power $\left(P_{S} / P_{J}\right)$. Multiple tone jamming is also effective against hybrid systems [33]. 


\subsubsection{ON-OFF Jammer}

The ON-OFF jammer (pulsed noise jammer) transmits a pulsed band limited Gaussian noise signal whose power spectral density just covers the spread spectrum system bandwidth $W$. The duty factor (the fraction of time during which the jammer turns on) for the jammer is denoted by $\rho$. The received jammer power spectral density is $P_{J} / \rho W$. This pulsed technique can also be used for single tone, multiple tone and partial band jammers.

\subsubsection{Repeater Jammer}

A repeater jammer receives the spread spectrum signal, distorts it in some well defined manner, and retransmits the signal at high power. The spread spectrum receiver then receives the distorted signal at high power and it will track and demodulate this distorted signal. However, there are two main issues that should be considered for this jammer. Firstly, the repeater jammer must distort the spread spectrum signal or else the jammer will act as a power amplifier for the desired signal. Secondly, receiving and transmitting simultaneously in the same band of frequencies presents formidable practical problems for the jammer.

\subsubsection{Smart Jammer}

For the jammer to be most effective, the jamming signal must be adapted to the spread spectrum system and to the actual received signal power. A jammer which has knowledge of the type of signaling being used, which can accurately predict the received signal power, and which can adapt to transmit the optimum jamming signal is called a smart jammer. A smart jammer is usually assumed in all worst case designs. 


\subsection{Motivation}

This research is sponsored and directed by the Egyptian Armament Authority of the Egyptian Ministry of Defense, and therefore the spread spectrum system that we focus on is intended for military applications. Because FHSS is widely used in military applications, the sponsor was interested in making this research in that direction. This wireless communication system will be used to transmit and receive data in battlefields, where the communication system is likely to be jammed by the enemy. The channel model in the battlefield is considered to be a frequency selective fading channel. That means the transmission suffers from jamming attacks and the channel effects. We study the case of two-way single-user communication between a command post and a soldier or a remote sensing device in the presence of jamming and channel effects, as shown in Fig. 1.1.

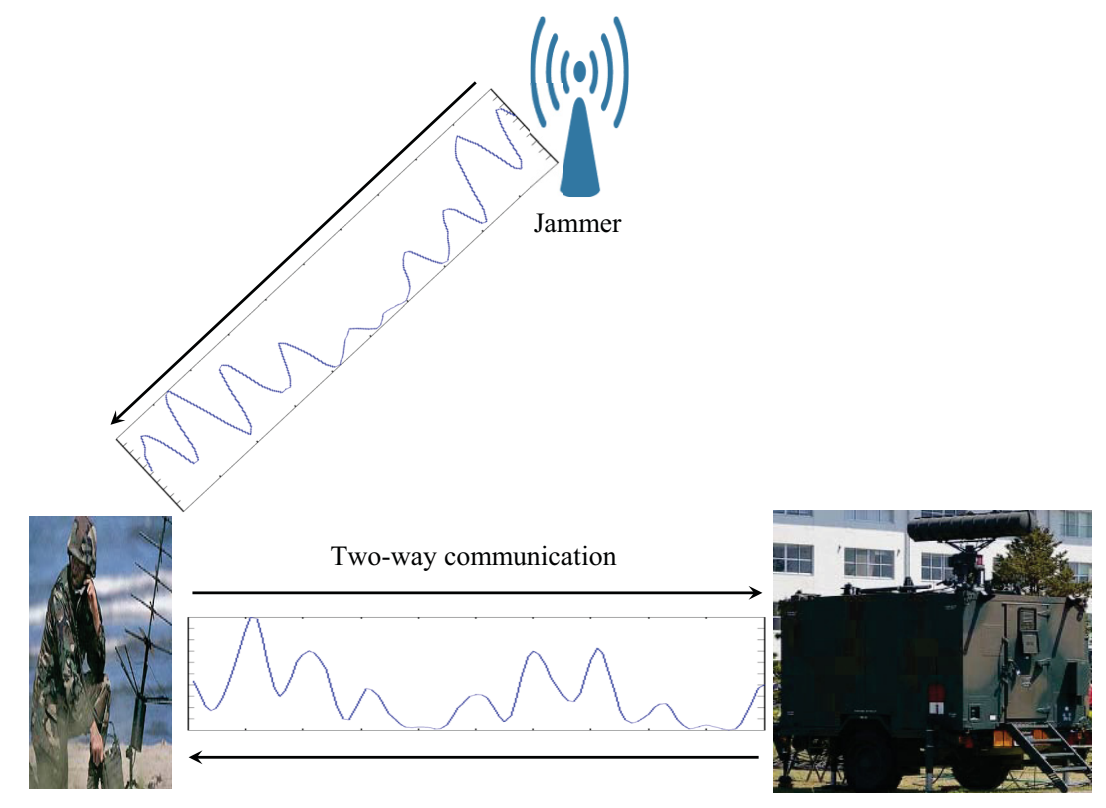

Figure 1.1: Two-way communication in the presence of jamming in frequency selective fading channel. 
There are many frequency hopping schemes that have been used to mitigate jamming, but they suffer from fading in frequency selective fading environments. On the other hand, there are some other schemes that mitigate fading effects, but they perform worse in jamming environments. In other words, the selection of frequency hopping tones is a trade-off between selecting faded tones to avoid jammed bands or selecting jammed tones to avoid faded bands. In this thesis we want to improve the existing techniques by proposing novel frequency hopping schemes that avoid jammed and faded bands and selecting the best available tones in the channel.

\subsection{Literature Review}

Frequency hopping spread spectrum is extensively analyzed in terms of the bit error rate for different channel models and jamming scenarios using different receiver structures. In [34] the performance of FH/BFSK is analyzed in terms of bit error rate $(\mathrm{BER})$ in the presence of wideband and partial band jamming (PBJ) in Rayleigh and Nakagami channel fading models. The performance of non-coherent FFH/BFSK in the presence of PBJ and in the absence of fading is examined in [35]. The BER performance of $\mathrm{FH} / \mathrm{MFSK}$ in the presence of PBJ and multi-tone jamming (MTJ) assuming a noise free channel is examined in [36]. Coherent detection for frequency hopping using $M$-ary frequency shift keying (MFSK) and $M$-ary phase shift keying (MPSK) in the presence of PBJ and MTJ is analytically studied [37], [38]. The BER performance of uncoded and coded noncoherent FH/MFSK is also examined in the presence of MTJ [39]. The repeater jamming and the limitations on its effectiveness against frequency hopping systems are described in [40].

FHSS is also used to detect and mitigate different jamming scenarios by suppressing or removing jamming sub-bands after detection using various methods and 
hopping over the remaining sub-bands randomly, but in all of these anti-jamming methods the authors did not consider interference or channel fading. Jamming recognition and suppression algorithms based on digital signal processing are used to intelligently mitigate jamming [41]. Jamming detection and cancelation is also achieved by using a two-element antenna array and maximum likelihood (ML) detection in a slow frequency hopping system [42], and it is shown that this technique provides much better performance than using beamforming. A novel receiver structure, named differential jamming rejection, is proposed in [43] to mitigate MTJ using fast frequency hopping. This technique provides better performance than using a linear combining receiver or a product combining receiver in terms of BER. In [44] the author provides a new jamming immunity indicator algorithm for jamming detection to discriminate between jamming and the desired signal. A new approach for jamming detection and classification using signal-processing-based electronic counter-countermeasures instead of using transmitter-based or receiver-based techniques is proposed [45].

There are two frequency hopping categories, channel ignorant frequency hopping spread spectrum (CIFHSS) and channel aware frequency hopping spread spectrum (CAFHSS). In CIFHSS the frequency hopping occurs over different sub-channels randomly without considering the channel impairments which degrades the communication system performance. Many improved receivers are designed for CIFHSS systems to enhance its performance in the presence of fading or jamming using different modulation and coding schemes. In [46] the authors proposed a new antijamming scheme named parallel jamming cancelation for slow CIFH/MFSK system. This two element antenna array scheme achieves better performance than maximum likely ML-based and traditional ML schemes over a wide signal-to-jamming ratio range. A new frequency hopping scheme named message driven frequency hopping 
(MDFH) is proposed, where a part of the transmitted signal is used to select the hopping pattern instead of the PN generator and that increases the spectral efficiency. The MDFH scheme is analyzed and further extended to be used with OFDM to enhance the spectral efficiency and also extended to mitigate jamming using cryptography [47], [48], [49], [50], [51]. In [52] a code-controlled message-driven frequency hopping scheme is proposed instead of using MDFH, where the hopping sub-channels are selected randomly using a small portion of the source information instead of using the majority of the transmitted signal as in MDFH. Coding is used in the receiver to retrieve the hopping pattern and to mitigate jamming without a priori knowledge and without the need for synchronization while keeping high spectral efficiency. Using non-coherent frequency hopping spread spectrum in conjunction with different modulation schemes such as minimum shift keying and duobinary minimum shift keying, and by using simplified Viterbi decoding is proposed to mitigate PBJ [53]. In [54] the authors suggested to use a self-normalized non-coherent receiver with fast $\mathrm{CIFH} / \mathrm{MFSK}$ and to increase the modulation order to protect the transmission against PBJ in fading channels. This system has better performance than conventional FFH/MFSK. In [55] the authors proposed two new modulation schemes, mMFSK and amMFSK, to be used with CIFH in fading channel. The proposed schemes provide better performance than conventional FH/MFSK. A maximum likelihood receiver is proposed to enhance the performance of $\mathrm{CIFFH} / \mathrm{BFSK}$ in the presence of MTJ and it achieves better BER performance than a product combining receiver, a ratio-static-combining receiver and a self-normalized receiver [56]. Turbo decoding and non-orthogonal continuous phase frequency shift keying (CPFSK) when used with non-coherent $\mathrm{CIFH}$ are robust in frequency selective fading channels in the presence of jamming [57]. 
In CAFHSS, which is also named adaptive frequency hopping, the channel characteristics are monitored first and then the receiver detects the best available subchannels and assigns these sub-channels to the transmitter for usage. This category of frequency hopping is used in multi-user scenarios that assign the sub-channels with the best channel gains for different users to achieve the best performance. A new adaptive frequency hopping scheme that adapts the tone separation to get the optimum spectral efficiency in fading channel and in multiuser scenarios is proposed [58]. Dynamic frequency hopping (DFH) is also proposed to assign different sub-channels to different users based on the signal-to-interference ratio (SIR). It achieves much better spectral efficiency and supports more users than random frequency hopping [59], [60]. Another technique called interleaved frequency hopping $(\mathrm{IFH})$ is proposed that increases the diversity of hopping tones and it increases the capacity in multiuser systems [61]. A simplified multi-user detector is proposed for non-coherent FFH/MFSH in multiple access channels [62]. Rake receivers and interference cancelation are used in CDMA systems in conjunction with adaptive slow frequency hopping $(\mathrm{SFH})$ to enhance the BER performance over fading channels [63]. Another adaptive SFH scheme that selects hopping tones which have the highest channel gains is proposed for CDMA systems that suffer from fading [64], [65]. Multiple access interference (MAI) is mitigated and spectral efficiency is improved by using a linear decorrelating detector with $\mathrm{AFH}$ in MC-CDMA system. This system provides better BER performance than systems using the water filling algorithm for channel allocation, systems using maximum ratio combining, and systems using matched filters [66].

CAFHSS is also used in single user scenarios to mitigate channel fading by assigning sub-channels with highest signal-to-noise ratio (SNR) or sub-channels with 
highest gains to the user's transmitter and the transmitter use these sub-channels to modulate the transmitted signal in a random fashion. However all of these CAFHSS schemes do not consider the presence of jamming. A new dynamic adaptive frequency hopping (DAFH) scheme is proposed in [67], [68]. The main idea behind this scheme is to assign all the hopping tones to the user and measure the packet error rate (PER). If the PER is higher than a specific threshold the hopping set is divided into two halves and the user randomly select one half of the hopping set to modulate the transmitted signal and the PER is again measured. If the PER is still higher than the threshold the system continue dividing the hopping set until the PER becomes lower than the threshold. Set doubling (joining two hopping sets together) is used if the threshold of the PER is lower than the threshold. This scheme has better throughput performance than adaptive frequency hopping. Instead of using a conventional adaptive frequency hopping scheme, the authors in [69] proposed to divide the total frequency band into many hopping sets and measure the PER of each hopping set and classify them as either good or bad hopping sets based on a predetermined threshold. Then they applied the moving average (MA) technique to the tones in bad sets to detect the tones that are not interfered. This scheme has better PER than conventional adaptive frequency hopping. The authors in [70] proposed to enhance the BER performance of high frequency communication in frequency selective fading channel by using adaptive frequency hopping. Matched frequency hopping (MFH), clipped matched frequency hopping $(\mathrm{CMFH})$ and advanced matched frequency hopping $(\mathrm{AMFH})$ are used to select hopping tones with high gains from the available frequency band in slowly fading dispersive channel [71], [72], [73]. 


\subsection{Contributions}

All frequency hopping schemes that are used to mitigate interference and fading usually use uncoded communication, and the BER metric is used to compare the performance of these systems. When it comes to comparing frequency hopping systems in the presence of jamming, all uncoded systems have unreasonably high bit error rates if any of the tones in the hopping set are jammed. It is therefore typically necessary to use some form of error control coding to recover data bits that are lost due to jamming. The more important and relevant measure in this case is to consider how much coding we need (i.e. what code rate we should use) to have a robust frequency hopping system to mitigate jamming in a specific channel model. We therefore measure the system performance in terms of the average throughput that can be realized with a rate-adaptive coded system.

A type-II hybrid automatic repeat request (ARQ) scheme with incremental redundancy is used in this frequency hopping system to achieve reliable data transmission. In this ARQ scheme the receiver sends an acknowledgement to the transmitter if the data is received correctly and this acknowledgement should be received by the transmitter within a specific period of time. If the transmitter does not receive the acknowledgement from the receiver, it will transmit additional parity bits until the receiver sends the acknowledgement back.

We assume that a capacity-achieving code is used for error correction, which can be approximated by either a family of rate-compatible fixed-rate codes or, more practically, a rate-adaptive code such as a Raptor code [74], used in conjunction with an incremental redundancy ARQ scheme.

We attempt in this thesis to improve battlefield signal transmission using new 
adaptive frequency hopping spread spectrum schemes that are desired to mitigate interference and jamming in frequency selective fading channels. Different existing frequency hopping schemes such as random frequency hopping (RFH), matched frequency hopping $(\mathrm{MFH})$, clipped matched frequency hopping $(\mathrm{CMFH})$, and advanced frequency hopping $(\mathrm{AFH})$ will be presented and compared. We propose to use the $\mathrm{MFH}, \mathrm{CMFH}$ and $\mathrm{AFH}$ as anti-jamming techniques and we also propose to optimize their control parameters to enhance their performance in jammed frequency selective fading channels. We also propose new random frequency hopping techniques that combine the advantages of randomness and adaptivity of frequency hopping and optimize their parameters to enhance their performance. The following papers were published based on the research leading to this thesis:

- W. M. Saad and I. Marsland, "Jamming and fading channels mitigation using anti-jamming advanced frequency hopping," International Conference on Electrical and Computer Systems, Ottawa, Aug. 2012.

- W. M. Saad and I. Marsland, "Weighted random frequency hopping in the presence of narrowband interference," Electrical Engineering/Electronics, Computer, Telecommunications and Information Technology Conference (ECTICON 2013), Krabi, Thailand, Jan. 2013.

\subsection{Thesis Outline}

The thesis consists of three parts. The first part discusses wireless communication challenges and their solutions, the different frequency hopping spread spectrum schemes and how their algorithms select the hopping tones, and then we will compare all these schemes in terms of throughput in the absence and presence of jamming in 
frequency selective fading channels. The second part proposes to use MFH, CMFH and $\mathrm{AFH}$ to reduce the detrimental effects of jamming in communication systems and proposes to optimize their performance in the presence of jamming. The third part provides new RFH schemes that enhance the performance of random frequency hopping in the presence of smart jamming in frequency selective fading channels.

In Chapter 2 we investigate wireless communication needs, limitations, challenges, impairments and previous solutions. We discuss the fundamentals of the frequency hopping spread spectrum technique, and provide the basic concepts, capabilities and properties of these systems. We present previous spread spectrum schemes and discuss their advantages.

In Chapter 3 we compare the performance of all the presented existing frequency hopping schemes in terms of throughput in the absence and presence of jamming in frequency selective fading. We also optimize these frequency hopping schemes and compare their optimized performance.

In Chapter 4 we propose new random and adaptive frequency hopping spread spectrum schemes. The new schemes select the hopping frequencies according to the frequency response of the channel. The new schemes use the same random generators that are used in RFH scheme. However, the generated hopping patterns have better transmission performance than $\mathrm{RFH}$ in jammed wireless communication channels.

In Chapter 5 we summarize the important results and comparisons of the new frequency hopping schemes and we present some topics for future work. 


\section{Chapter 2}

\section{Review of Frequency Hopping Spread Spectrum}

In the proposed research, we explore new methods for frequency hopping spread spectrum (FHSS) systems. These methods are better able to exploit the frequency

selective nature of the wireless channel, while providing greater resilience to jamming and other interference. In this chapter we provide a brief review of FHSS in general, and describe some more recently proposed FHSS hopping set selection algorithms.

\subsection{Frequency Hopping Spread Spectrum}

Frequency hopping is a spread spectrum technique that involves partitioning the allocated frequency band, called the hopping band, into a large number of smaller sub-bands. These sub-bands are also called carrier frequencies, channels, tones, subchannels, or sub-carriers. Transmission is carried out in short bursts on one sub-band at time, hopping from sub-band to sub-band in a pseudo-random fashion after each burst, as illustrated in Fig. 2.1. The figure illustrates how the total system bandwidth of $B_{t o t} \mathrm{~Hz}$ is divided into $K$ narrow sub-bands, where each sub-band has a bandwidth of $B_{s b}=B_{t o t} / K \mathrm{~Hz}$. The system uses one sub-band at a time, for a hop duration of $T_{s b}$ seconds, before hopping to another sub-band. In this manner all sub-bands are used roughly an equal amount of time, but no sub-band is used continuously for a long time. 


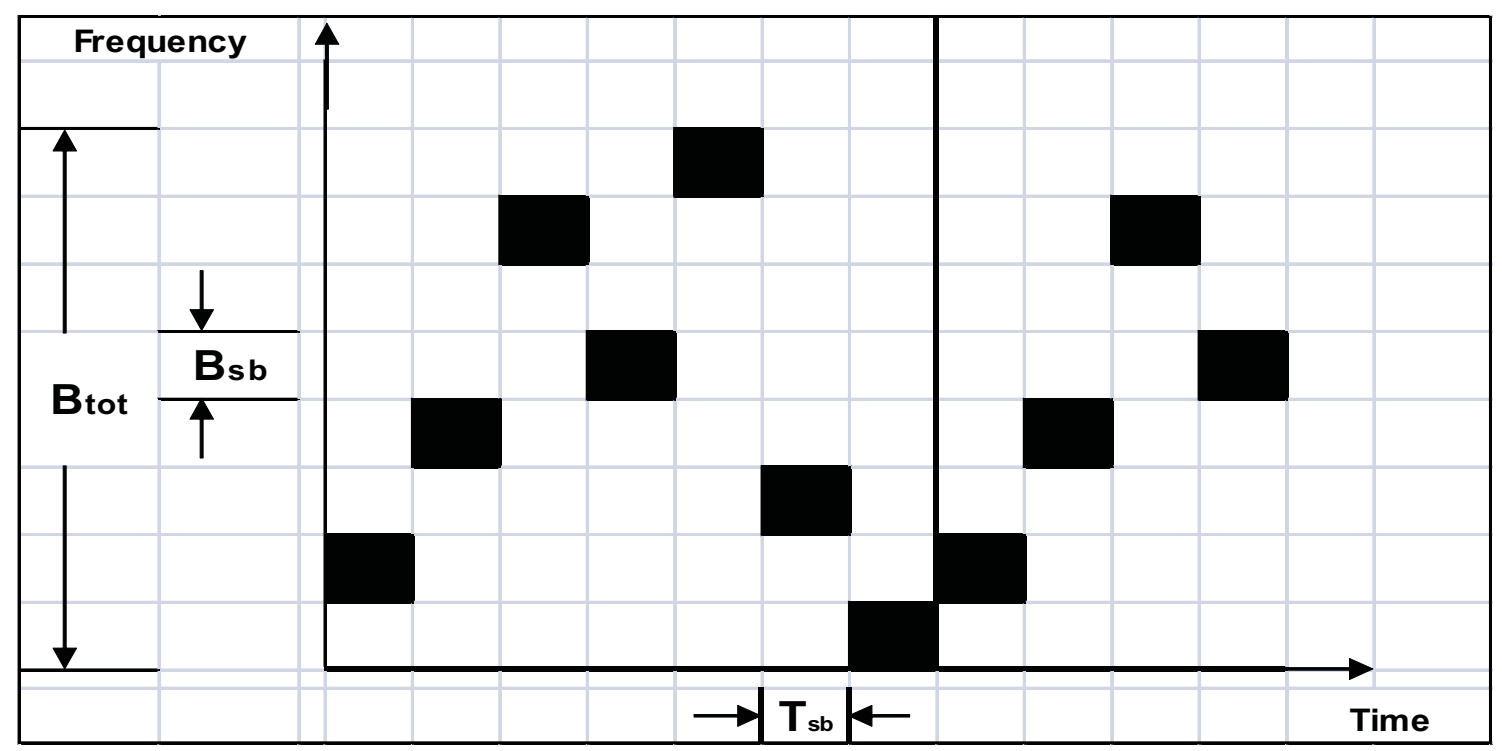

Figure 2.1: Frequency hopping patterns.

The rate at which the hops occur, relative to the symbol transmission rate, allows us to categorize the FHSS system as either fast or slow hopping [27]. If the hop rate, which is the inverse of the hop duration, is greater than the symbol rate, then the system is characterized as fast hopping. In this case each transmitted symbol is divided over multiple sub-bands. Slow hopping occurs when the hop rate is less than or equal to the symbol rate, which means that one or more data symbols are transmitted within each hop.

The order in which the hopping occurs over the sub-bands is called the hopping pattern. This sequence is generated by a secure pseudo-random code generator at the transmitter. The hopping pattern is also known by the intended receiver so it can easily recover the transmitted signal, but other receivers, without this knowledge, are unable to detect the signal, thereby impeding undesirable signal interception and making intentional jamming more difficult. To provide a secure and unpredictable frequency hopping pattern, the pattern should be a random sequence and this sequence 
should have a large period. The large period prevents the capture and storage of a period of the pattern by a jammer or eavesdropper.

A simplified block diagram of a FHSS system is shown in Fig. 2.2. The message bits are used to first generate a modulated intermediate frequency (IF) signal. Any basic modulation scheme, such as phase shift keying (PSK), amplitude shift keying (ASK), or frequency shift keying (FSK), could be used in theory, but FSK is the most widely used in FHSS systems. The IF signal is mixed with the frequency synthesizer output to produce the transmitted radio frequency (RF) signal. The frequency synthesizer generates the RF carrier signal with a frequency based on the output of code generator. Because the code generator output changes every $T_{s b}$ seconds, so does the carrier frequency, causing the frequency hopping in the transmitted RF signal.

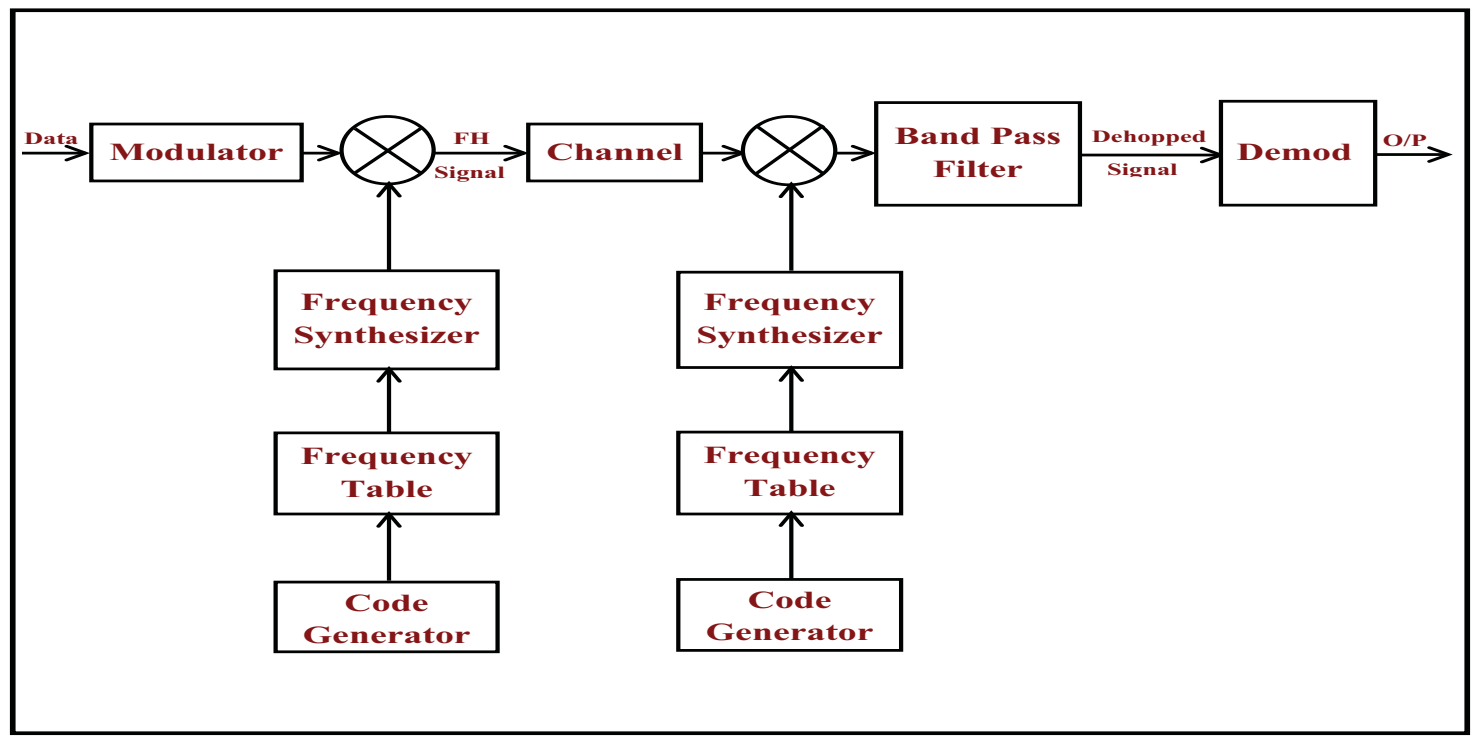

Figure 2.2: Block diagram of a frequency hopping system.

At the receiver, the received $\mathrm{RF}$ signal is down-converted to IF by mixing it with the output of another frequency synthesizer. The frequency generator must 
be driven by a code generator that produces the same hopping pattern as used at the transmitter, and temporal synchronization must be maintained so that frequency hopping occurs at the same time as in the received signal. If the frequency hopping is sufficiently slow, it is possible to also maintain carrier synchronization, but this is not practical with fast frequency hopping, or even with slow frequency hopping when the hop duration only spans a few symbol intervals. As a result, noncoherent detection is typically employed with FHSS systems. The dehopped received IF signal is then demodulated to baseband and a traditional detector is used to recover the transmitted data.

In this thesis we focus on FHSS as a frequency selective fading and jamming mitigation technique. We consider slow hopping with noncoherently detected binary FSK. Although frequency hopping provides no advantage against white noise, it has good immunity to interference, frequency selective fading, and the near-far problem [27], [75], [76]. It is also less susceptible to jamming [14] and the transmitter and receiver are easy to implement with low power consumption and inexpensive components.

Since each sub-band is equally likely to be used in any given hop, and the subbands are selected randomly, we refer to this traditional frequency hopping scheme as random frequency hopping (RFH). In $\mathrm{RFH}$, the random generator generates a random code to a fixed frequency table, that assigns each code to a specific frequency regardless of the channel characteristics. The generated random frequency is used to modulate the transmitted signal as shown in Fig. 2.2. One problem with RFH is that, in a frequency-selective fading environment, at any given time some sub-bands may be severely attenuated (or, in a jamming environment, some sub-bands may be jammed). Better performance is possible if frequency hopping is performed over only 
a subset of the sub-bands. Sub-bands that have severe attenuation or are jammed are excluded from the subset, which is called the hopping set. In the following section we discuss some previously proposed algorithms for selecting the hopping set.

\subsection{Hopping Set Selection Algorithms}

Frequency hopping schemes that involve monitoring the variation of the channel characteristics, and then selecting the hopping set according to these characteristics, are referred to as channel-aware frequency hopping schemes, and generally provide better performance than the channel-ignorant RFH scheme. In the selection of the hopping set, these schemes try to avoid the sub-bands that have low channel gains or are jammed. This avoidance-type of frequency hopping enhances signal transmission, but there is a trade-off between selecting sub-bands with high channel gains and avoiding jammed sub-bands. This is because sub-bands with high gain tend to be adjacent, making them easier to jam. The performance of the channel-aware schemes is better than the channel ignorant schemes in the presence of interference and jamming [77].

Many techniques have been proposed for selecting the hopping set, including highest gain frequency hopping (HGFH), matched frequency hopping (MFH), clipped matched frequency hopping $(\mathrm{CMFH})$, and advanced frequency hopping $(\mathrm{AFH})$. In this section, all these algorithms will be presented and explained.

\subsubsection{Highest Gain Frequency Hopping}

In HGFH, the channel frequency response is first monitored and then the hopping subset is selected as the $M$ sub-bands that have the highest channel gains. As an illustrative example, Fig 2.3 shows the selected 15 sub-band hopping set for one 
realization of a WINNER frequency selective fading channel [78] consisting of 100 sub-bands. By only hopping over the best sub-bands, HGFH can provide excellent throughput. However, as the channel frequency response changes over time, the subbands in the hopping subset may become worse, degrading the throughput until the receiver can determine a new hopping subset. Furthermore, because the selected sub-bands tend to be adjacent (or in a small number of tightly spaced clusters), it is easy for a jammer to jam most of the selected sub-bands. It is therefore preferable to select sub-bands that are dispersed, even if this means not necessarily selecting the best sub-bands.

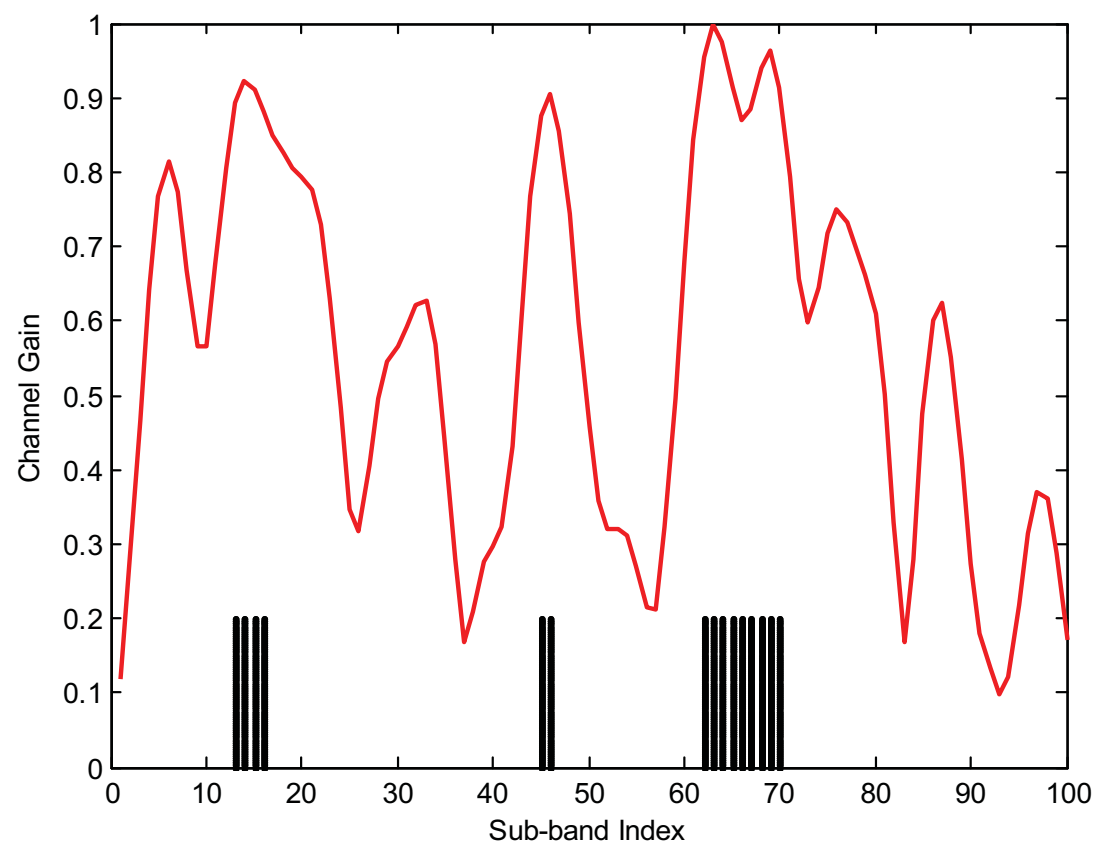

Figure 2.3: Example of a HGFH hopping set where $M=15$ sub-bands are selected out of $K=100$. Vertical black lines indicate selected sub-bands.

\subsubsection{Matched Frequency Hopping}

The desire for frequency dispersion in the selected hopping set to avoid severe degradation caused by jamming led to the search for other hopping set selection 
algorithms. In the MFH scheme the $M$ sub-bands are selected based on the complex sub-bands channel gains, $\left\{H_{k}\right\}$, so that good sub-bands tend to be selected while also keeping the sub-bands reasonably well-spaced [71]. In particular, the normalized sub-band power metric,

$$
P_{k}=\frac{Q_{k}}{\sum_{l=1}^{K} Q_{l}}
$$

is calculated for each sub-band, $k \in\{1,2, \ldots, K\}$, where $Q_{k}=\left|H_{k}\right|^{2}$. Also calculated are the cumulative metrics

$$
C_{k}=\sum_{n=1}^{k} P_{n}
$$

which is a monotonically increasing function of $k$, spanning the range $[0,1]$. The cumulative metrics are used with the $M$ equally-spaced values over $[0,1]$ given by

$$
y_{m}=\frac{1}{M}\left(m-\frac{1}{2}\right) \quad m \in\{1,2, \ldots, M\}
$$

to determine the indices of the selected sub-bands. The index of the $m^{\text {th }}$ selected sub-band is given by the value of $k$ such that $C_{k-1} \leq y_{m}<C_{k}[71]$.

The steps of selecting the MFH hopping set are illustrated in Fig. 2.4, which illustrates how $M=15$ sub-bands are selected out of $K=100$ sub-bands using this procedure with a simple channel model. Fig. 2.5 illustrates the hopping set using the MFH algorithm in a sample realization of the WINNER channel model. It is clear that the algorithm tends to select sub-bands with low attenuation, while avoiding selecting clusters of adjacent sub-bands to avoid interference and jamming, as would be the case if one were to merely select the $M$ sub-bands with the highest gains. 


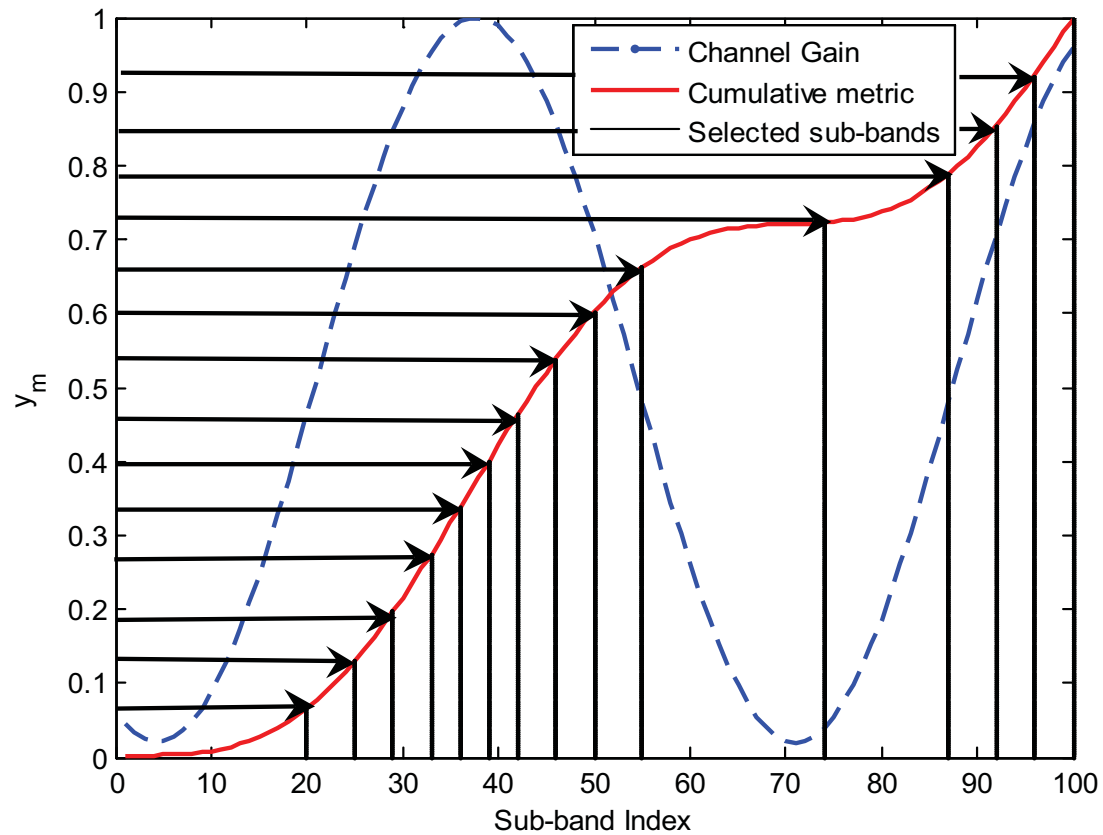

Figure 2.4: Illustration of selecting $M=15$ sub-bands out of $K=100$ using $\mathrm{MFH}$ in a simple channel.

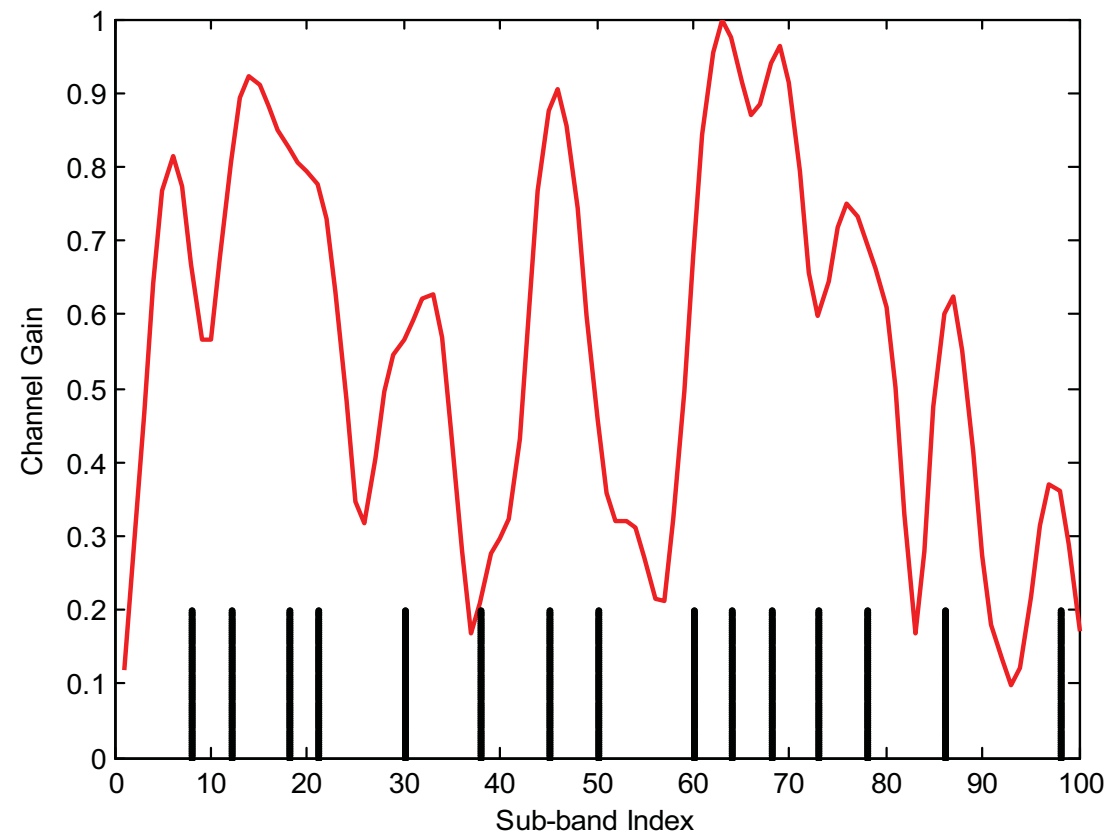

Figure 2.5: MFH hopping set of $M=15$ sub-bands for a sample channel realization. 
Although in MFH good sub-bands tend to be selected while keeping the subbands reasonably well-spaced, in some cases when the channel gains increase suddenly MFH selects the same sub-band more than once and that makes it more vulnerable to jamming attacks.

\subsubsection{Clipped Matched Frequency Hopping}

The clipped matched frequency hopping $(\mathrm{CMFH})$ scheme is an evolution of the MFH scheme [72]. The channel gains, $\left|H_{k}\right|^{2}$, are first clipped with a certain value, $T \max \left|H_{k}\right|^{2}$, proportional to the maximum gain, and then the clipped channel gains are decreased by this amount. That means any channel gain less than or equal to $T \max \left|H_{k}\right|^{2}$ is set to zero. The clipped channel gains are given by

$$
Q_{k}= \begin{cases}\left|H_{k}\right|^{2}-T \max \left|H_{k}\right|^{2} \text { if }\left|H_{k}\right|^{2}>T \max \left|H_{k}\right|^{2} \\ 0 & \text { if }\left|H_{k}\right|^{2}<T \max \left|H_{k}\right|^{2} .\end{cases}
$$

The MFH technique is then applied to the new clipped channel gains. The CMFH hopping subset is shown in Fig 2.6, using $M=15, K=100$, and $T=0.2$. The CMFH scheme will result in more concentrated sub-bands with lower attenuation than in the MFH scheme.

\subsubsection{Advanced Frequency Hopping}

The performance of the MFH and CMFH schemes are improved by using the AFH scheme [73]. The AFH scheme further improves the performance by selecting more sub-bands with high gain. It uses an optimization function given by

$$
Q_{k}=\frac{\left|H_{k}\right|^{2}}{(1+\alpha) \max \left|H_{k}\right|^{2}-\left|H_{k}\right|^{2}}
$$




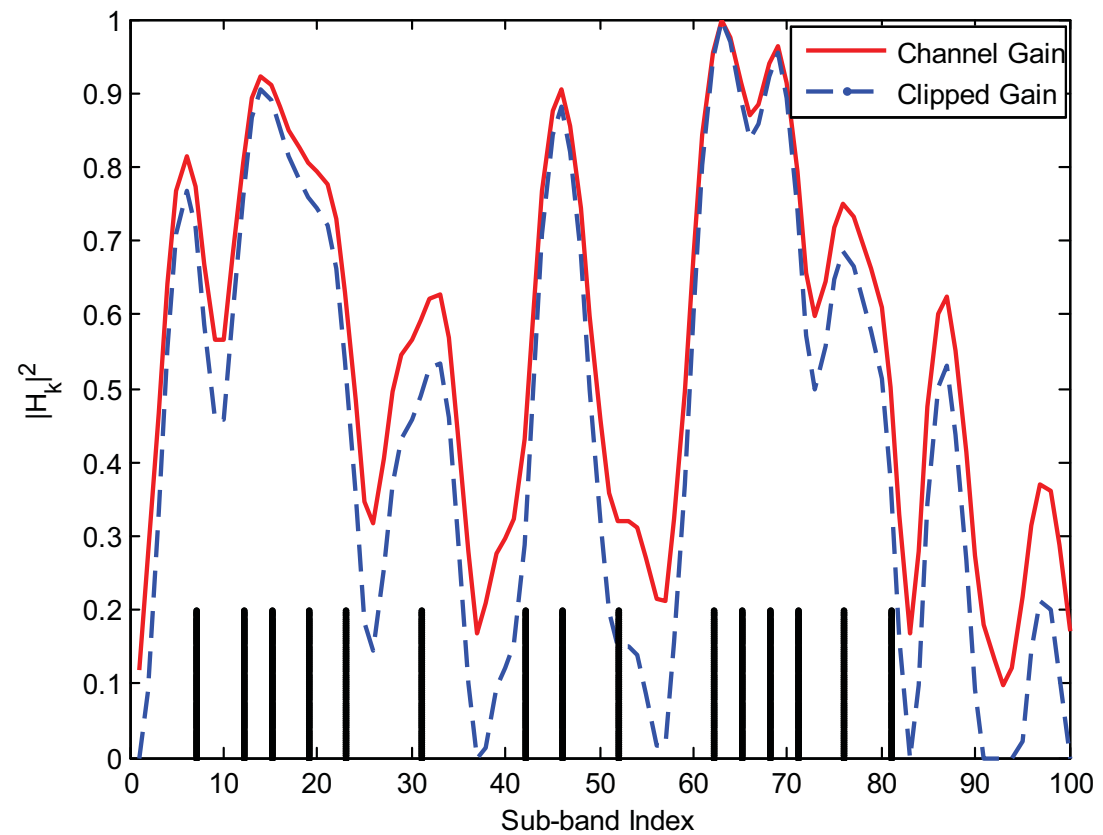

Figure 2.6: $\mathrm{CMFH}$ hopping set with $M=15$ sub-bands.

where $\alpha$ is a small value. After calculating $Q_{k}$ for each sub-band, using $\alpha=0.01$, the $M$ sub-bands will be selected in the same way as the MFH scheme. This will result in sub-bands with high attenuation being avoided as shown in Fig 2.7.

\subsection{System Model}

A block diagram of the operation of the system with adaptive PBJ is shown in Fig 2.8. The transmitter begins by transmitting pilot signals over all the available sub-bands so the receiver can estimate the sub-band gains. By applying one of the hopping set selection algorithms described in Section 2.2, the receiver determines the hopping set, which is passes back to the receiver. The transmitter begins transmission by hopping pseudo-randomly over the tones in the hopping set. With its knowledge of the hopping set and the hopping sequence, the receiver is able to detect the transmitted information. Periodically, as the channel changes, the transmitter 


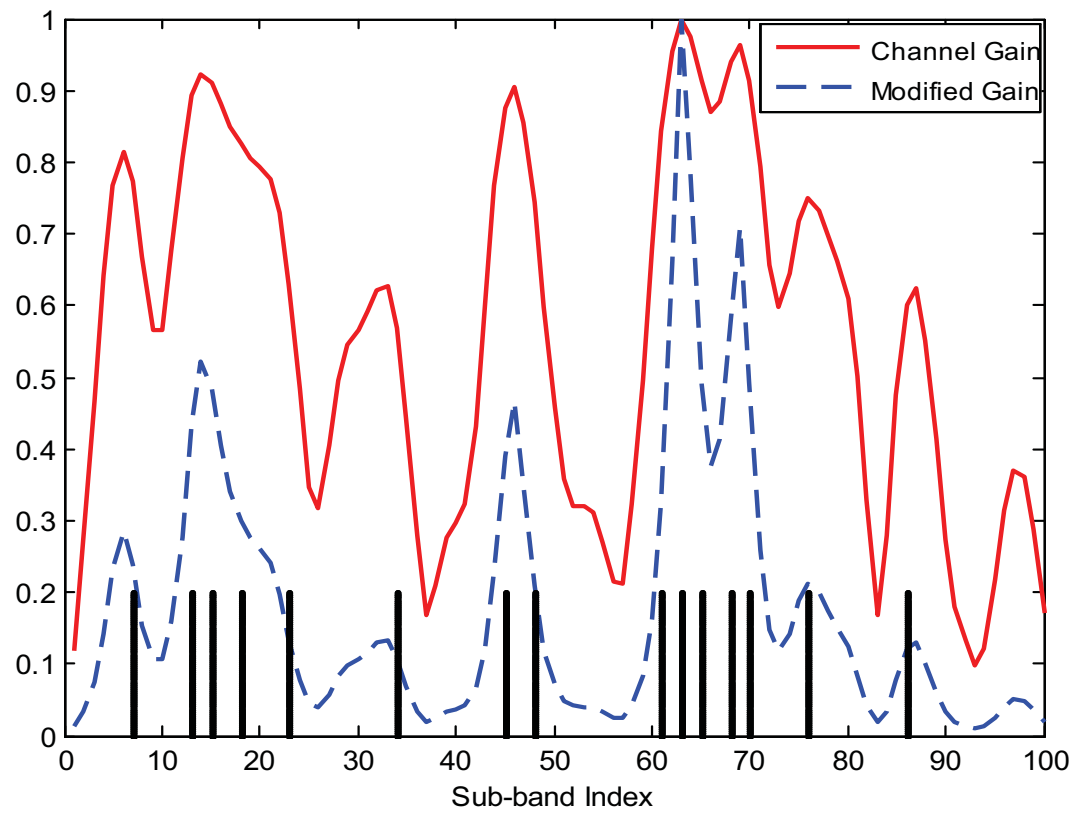

Figure 2.7: AFH hopping set of $M=15$ sub-bands.

retransmits pilot signals and the receiver determines a new hopping set. Transmission then moves to the new hopping set. While transmission is taking place, an adaptive jammer scans all the available channels and detects which ones are being used, thereby gaining knowledge of the hopping set, but not the hopping sequence. We assume the presence of adaptive PBJ, where the jammer concentrates all its available power to jam a fraction of the transmission bandwidth [77]. The jammer simultaneously jams two disjoint clusters of adjacent sub-bands, each containing $5 \%$ of the transmission bandwidth. With adaptive PBJ the jammer selects the two jamming clusters so that as many of the selected hopping frequencies as possible are jammed. The jammer continues attacking the selected sub-bands until it detects a new hopping set, when it then repositions the two jamming clusters to again attack the largest number of selected sub-bands.

We use a frequency selective fading WINNER channel model to model the fre- 


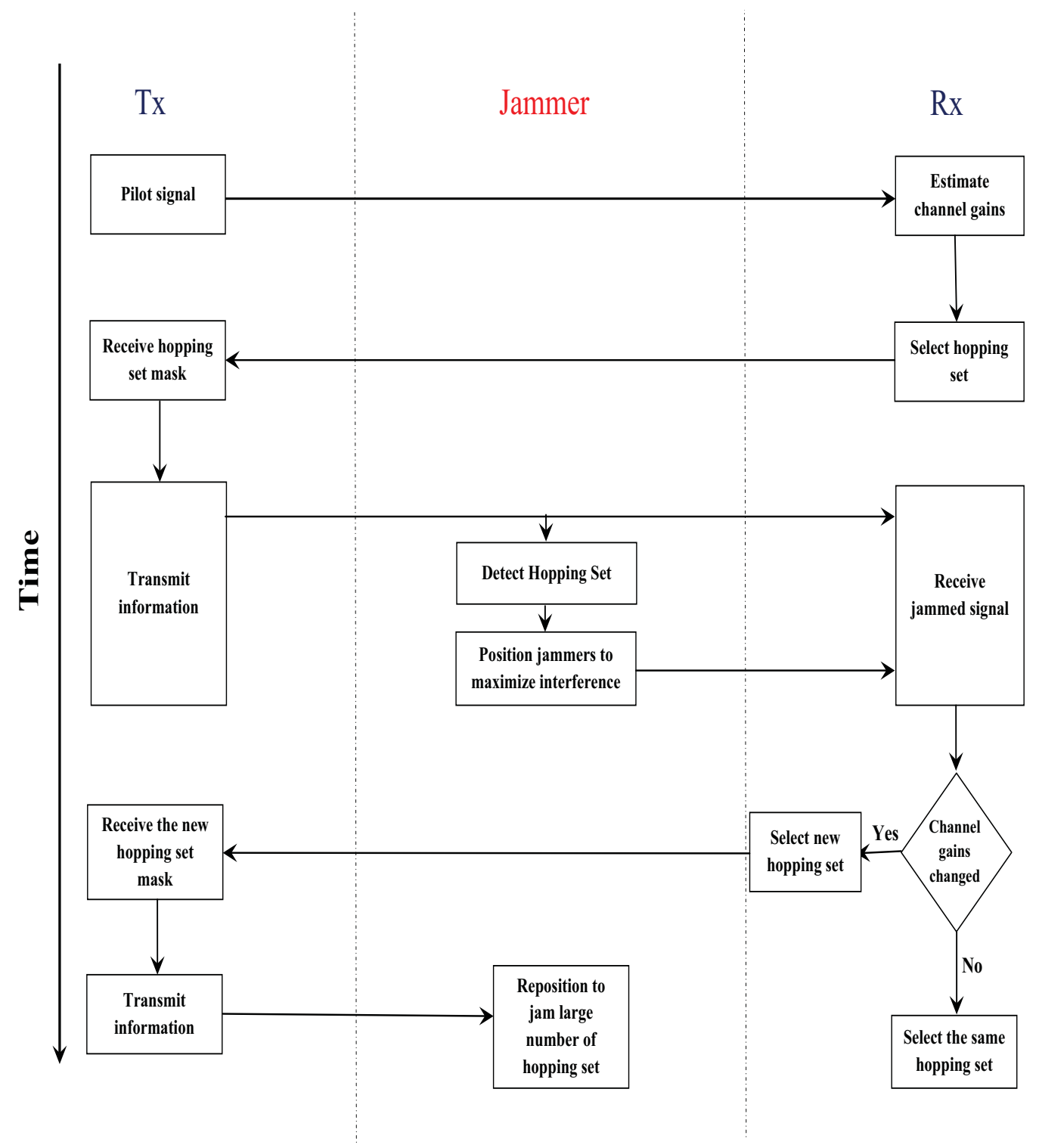

Figure 2.8: System model flowchart.

quency selective fading channel [78]. The WINNER channel models cover from short range to wide area within a frequency range 2 to $6 \mathrm{GHz}[79]$. For our simulations we consider that the centre frequency of the $k^{t h}$ sub-band is

$$
f_{k}=f_{c}+\frac{W}{K}\left(k-\frac{K}{2}-\frac{1}{2}\right)
$$

for $k \in\{1,2, \ldots, K\}$. It is assumed that $K$ is sufficiently large so that the channel 
frequency response over each sub-band is essentially flat, but varies from sub-band to sub-band. The complex channel gain of the $k^{t h}$ sub-band is $H_{k}=H\left(f_{k}\right)$ where $H(f)$ is the frequency response of the broadband channel. The impulse response of the broadband channel is modeled as the combination of signal components in $L$ distinct clusters, so the impulse response is

$$
h(t)=\sum_{l=1}^{L} h_{l} \delta\left(t-\tau_{l}\right)
$$

where $\tau_{l}$ is the delay of cluster $l$, and $h_{l}$ is its complex gain, which is modeled as a zero-mean complex Gaussian random variable with a variance of $\sigma_{l}^{2}$. The frequency response of the channel is given by

$$
H(f)=\digamma\{h(t)\}=\sum_{l=1}^{L} h_{l} e^{-j 2 \pi f \tau_{l}}
$$

and the sub-band gains are

$$
H_{k}=\sum_{l=1}^{L} h_{l} \exp \left\{-j 2 \pi \tau_{l}\left[f_{c}+\frac{W}{K}\left(k-\frac{K}{2}-\frac{1}{2}\right)\right]\right\} .
$$

Because all uncoded frequency hopping systems have unreasonably high bit error rates if any of the sub-bands in the hopping set are jammed, it is typically necessary to use some form of error control coding to recover data bits that are lost due to jamming. We therefore measure the system performance in terms of the average throughput that can be realized with a rate-adaptive coded system. In particular, we assume that a capacity-achieving code is used, which could be approximated by either a family of fixed-rate codes or, more practically, a rate-adaptive code such as a Raptor code [74], used in conjunction with an incremental redundancy ARQ scheme. The modulation-constrained channel capacity of noncoherently detected binary FSK 


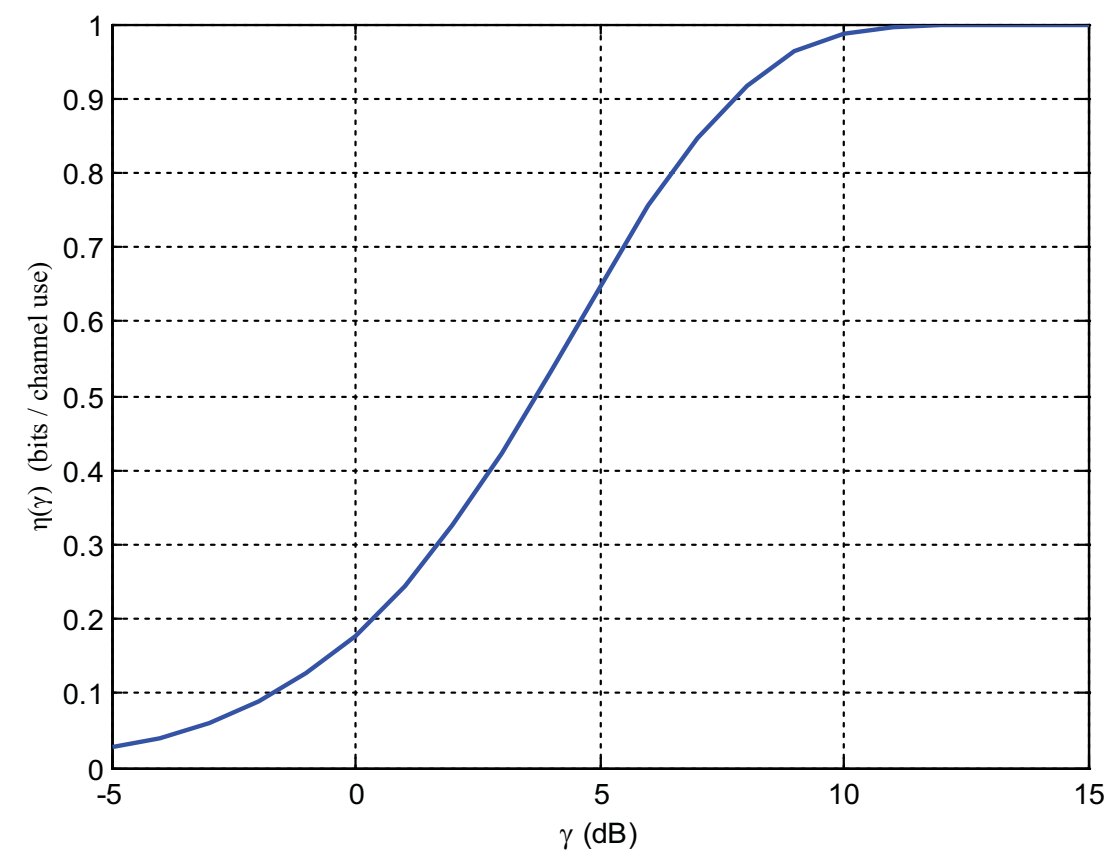

Figure 2.9: The modulation-constrained channel capacity of noncoherently detected binary FSK over the AWGN channel.

over an additive white Gaussian noise channel with a SNR of $\gamma$ given by [80]

$$
\begin{aligned}
\eta(\gamma)= & \int_{0}^{\infty} I_{0}\left(\beta r_{1}\right) \log _{2} I_{0}\left(\beta r_{1}\right) r_{1} \exp \left(-\frac{r_{1}^{2}+\beta^{2}}{2}\right) d r_{1} \\
& -\int_{0}^{\infty} \int_{0}^{\infty} \frac{1}{2}\left[I_{0}\left(\beta r_{1}\right)+I_{0}\left(\beta r_{2}\right)\right] \log _{2}\left(\frac{1}{2} I_{0}\left(\beta r_{1}\right)+\frac{1}{2} I_{0}\left(\beta r_{2}\right)\right) \\
& \times r_{1} r_{2} \exp \left(-\frac{r_{1}^{2}+r_{2}^{2}+\beta^{2}}{2}\right) d r_{1} d r_{2}
\end{aligned}
$$

where $\beta=\sqrt{2 \gamma}$ and $I_{0}(\cdot)$ is the modified Bessel function of the first kind. A derivation of (2.10) is included in Appendix A. Although there is no known closed form expression for $\eta(\gamma)$, it can easily be evaluated numerically, and is shown in Fig. 2.9.

To determine the throughput of a frequency hopping system over a frequency selective channel, we note that for a given channel realization, $\mathbf{H}=\left[H_{1}, H_{2}, \ldots, H_{K}\right]$, 
the throughput when sub-band $k$ is used is $\eta\left(\left|H_{k}\right|^{2} \gamma\right)$ where $\gamma$ is the average SNR. When RFH is used all sub-bands are equally likely to be selected, so the expected throughput for channel realization $\mathbf{H}$ is

$$
\eta(\mathbf{H}, \gamma)=\frac{1}{K} \sum_{k=1}^{K} \eta\left(\left|H_{k}\right|^{2} \gamma\right) .
$$

With MFH, CMFH and AFH, one of the $M$ sub-bands in the hopping set will be used with equal probability in each time slot, so the expected throughput is

$$
\eta(\mathbf{H}, \gamma)=\frac{1}{M} \sum_{m=1}^{M} \eta\left(\left|H_{k_{m}}\right|^{2} \gamma\right)
$$

where $k_{m}$ is the index of the $m^{t h}$ selected sub-band, which depends on the hopping scheme and $\mathbf{H}$.

In either case the average throughput, averaged over all channel realizations, is

$$
\bar{\eta}(\gamma)=E[\eta(\mathbf{H}, \gamma)]=\int_{\mathbf{H}} \eta(\mathbf{H}, \gamma) f(\mathbf{H}) d \mathbf{H}
$$

where $f(\mathbf{H})$ is the probability distribution of the channel realization. We can evaluate (2.13) numerically using Monte Carlo integration by randomly selecting a large number of channel realizations and averaging the throughput for each realization as given by (2.11) or (2.12) depending on the hopping scheme.

We consider the case where the jammer has finite power, so communication remains possible in jammed sub-bands, but with a lower throughput. The throughput therefore depends not only on the average SNR, $\gamma_{N}=\frac{E_{s}}{N_{0}}$, but also on the average signal-to-jamming ratio (SJR), $\gamma_{J}=\frac{E_{s}}{N_{J}}$, where $E_{s}$ is the transmitted signal energy (per bit), $N_{0}$ is the single-sided noise power spectral density (PSD) and $N_{J}$ is the single-sided jamming PSD in a jammed sub-band. The jamming is modeled as an 
additive Gaussian random process. Taking into account the channel gain between the transmitter and the receiver, $H_{S, k}$, and the gain between the jammer and the receiver, $H_{J, k}$, the instantaneous signal-to-jamming-and-noise ratio (SJNR) in the $k^{\text {th }}$ sub-band is

$$
\begin{aligned}
\rho_{k} & =\frac{\left|H_{S, k}\right|^{2} E_{s}}{\left|H_{J, k}\right|^{2} N_{J}+N_{0}} \\
& =\frac{\left|H_{S, k}\right|^{2}}{\frac{\left|H_{J, k}\right|^{2}}{E_{s} / N_{J}}+\frac{1}{E_{s} / N_{0}}} \\
& =\frac{\left|H_{S, k}\right|^{2}}{\frac{\left|H_{J, k}\right|^{2}}{\gamma_{J}}+\frac{1}{\gamma_{N}}} .
\end{aligned}
$$

The throughput if the sub-band is jammed is then $\eta\left(\rho_{k}\right)$, which is used in (2.11) or (2.12) to find the average throughput. If the sub-band is not jammed, the throughput is $\eta\left(\left|H_{S, k}\right|^{2} \gamma_{N}\right)$. 


\section{Chapter 3}

\section{Optimization of Frequency Hopping Schemes}

Although MFH, CMFH and AFH have previously been proposed and described in published literature, the performance of these schemes has not been adequately investigated and compared. In particular, there are three shortcomings to the published analysis that we hope to address. Firstly, the existing work only considers the BER

of uncoded systems as the performance metric, whereas we feel that the throughput of coded systems is a more relevant metric. Secondly, although these schemes are intended for use in jamming environments, their performance has only been studied in the absence of jamming. Thirdly, these schemes have only been investigated with one fixed set of parameters, with no attempt at parametric optimization. In this chapter we attempt to overcome these shortcomings and provide a more meaningful comparison between the systems, highlighting their strengths and weaknesses.

\subsection{Simulation Environment and Performance Metric}

For our simulations we consider the case where the total allocated system frequency band of $W=100 \mathrm{MHz}$, centered about $f_{c}=2.4 \mathrm{GHz}$, is divided into $K=100$ sub-bands. For the simulation results presented in this thesis we use the WINNER D1 channel model, suitable for rural non-line-of-sight applications, which is based on the parameters given in Table 3.1. There are $L=10$ clusters in this model, and the 
Table 3.1: WINNER D1 channel model parameters

\begin{tabular}{|c|c|c|c|c|c|c|c|c|c|c|}
\hline Cluster \# & 1 & 2 & 3 & 4 & 5 & 6 & 7 & 8 & 9 & 10 \\
\hline \hline Delay $(\mathrm{ns})$ & 5 & 0 & 5 & 15 & 20 & 25 & 55 & 100 & 170 & 420 \\
\hline Power $(\mathrm{dB})$ & -5.2 & -1.8 & -3.3 & -7 & -5.3 & -7.1 & -9 & -4.2 & -12.4 & -26.5 \\
\hline
\end{tabular}

table shows the delays, $\tau_{l}$, and power gains, $\sigma_{l}^{2}$, for each cluster. The power gains are normalized so that $\sum_{l=1}^{L} \sigma_{l}^{2}=1$.

\subsection{Performance of the Original Schemes}

The MFH, CMFH and AFH schemes, as originally proposed, have only been studied using $M=15, T=0.2$ and $\alpha=0.01$ as their parameters. In the next section we will investigate the effect of these parameters on the system performance, and find their optimal values. In this section, to provide a starting point for comparison, we will investigate the system performance in terms of average throughput, using just these originally proposed parameters. We first consider the case where there is no jamming, as was considered in the original studies, and then examine the effects of jamming on the performance.

As shown in Fig. 3.1, the performance of all schemes is examined in terms of average throughput given by (2.13). The results show that HGFH has the best performance, followed by $\mathrm{AFH}, \mathrm{CMFH}, \mathrm{MFH}$ and finally $\mathrm{RFH}$, which has the worst performance. To explain why this order occurs, it is useful to consider which subbands are selected by each of the different schemes. These are shown in Fig. 3.2 for a simple static frequency selective channel. We see that HGFH selects only the best sub-bands for the hopping set, so the best performance is delivered, but the selected sub-bands are tightly clustered. As we move down to AFH, CMFH and MFH we 


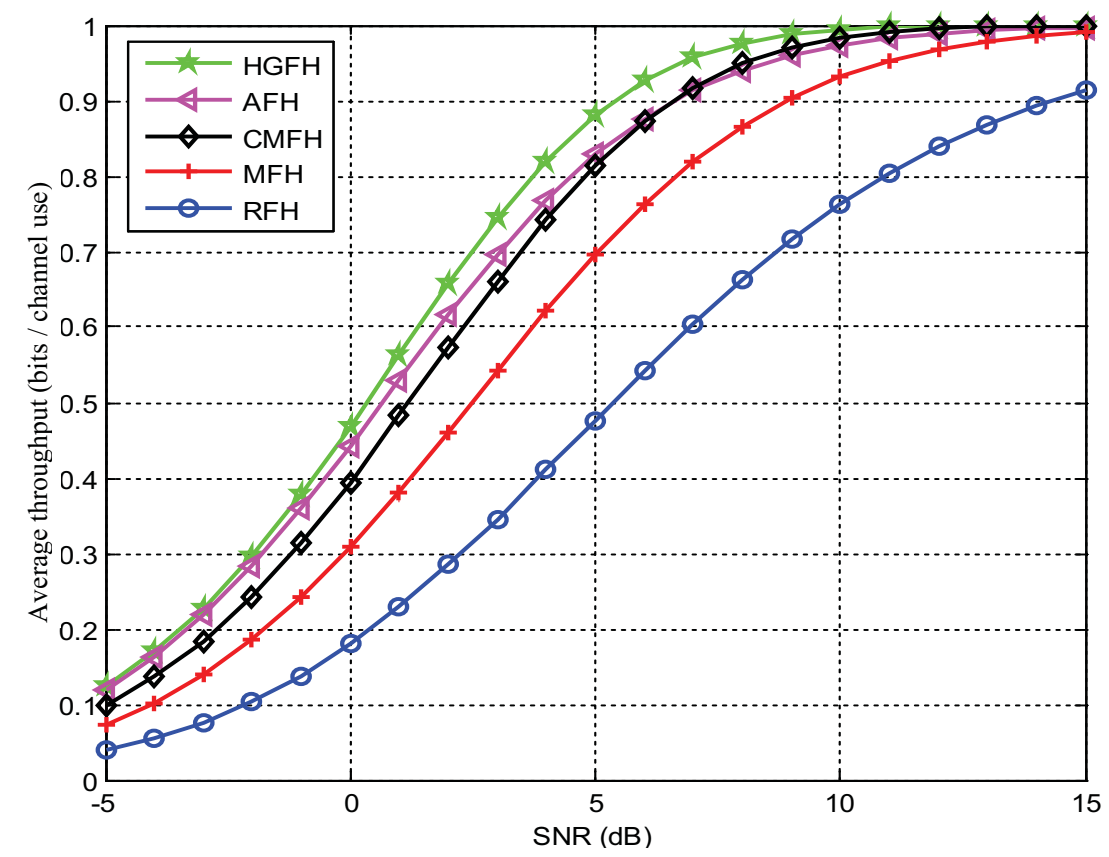

Figure 3.1: Average throughput performance of the frequency hopping schemes in the absence of jamming.

see that the selected sub-bands become more disperse, with a large number of more attenuated sub-bands being selected. For example, MFH selected sub-band 74, even though it is severely attenuated. Although RFH does not normally use a reduced-size hopping set, hopping instead over all sub-bands, we have shown a random selection of $M=15$ sub-bands to emphasize that RFH operates at the other extreme from HGFH, selecting sub-bands regardless of the channel gains, but providing a high degree of dispersion. As we will see, dispersion is useful for providing protection against jamming.

The average throughput of all the schemes in the presence of adaptive jamming is shown in Fig. 3.3. We note that the performance of all the schemes is degraded compared to their performance without jamming. More importantly, the results in the presence of adaptive jamming are nearly reversed, with HGFH giving the worst performance and RFH the best, particularly at high SNRs. The reason for this is that 

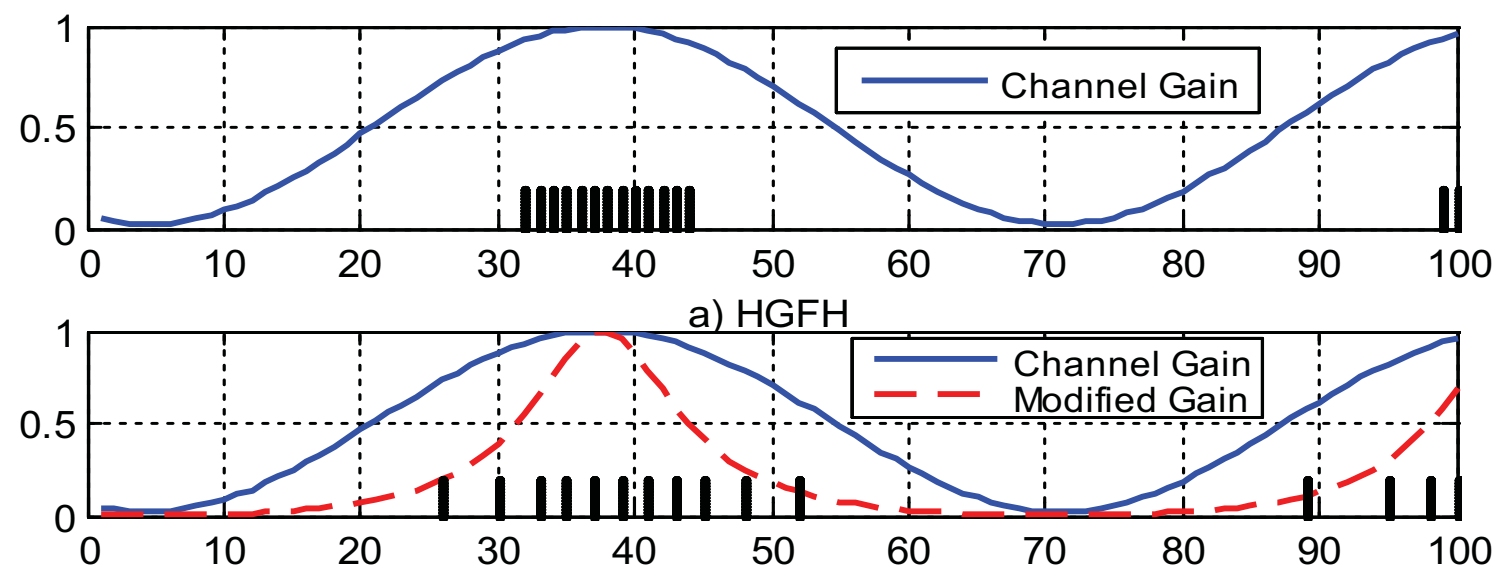

b) $\mathrm{AFH}$

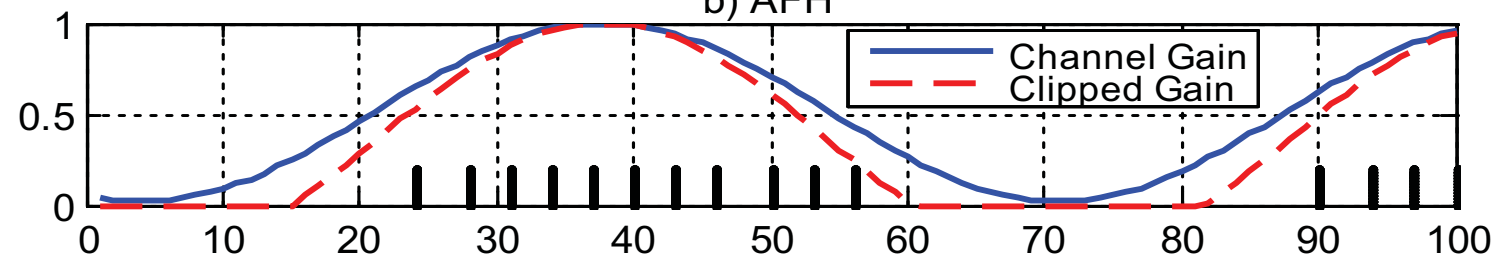

c) $\mathrm{CMFH}$

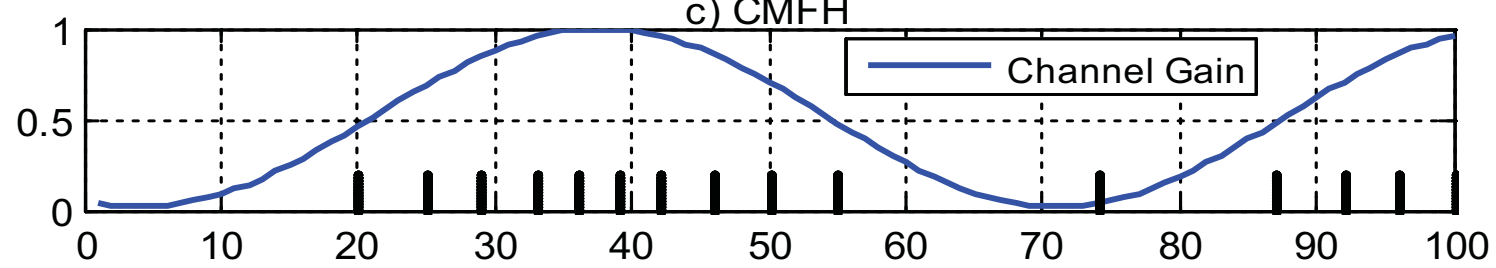

d) $\mathrm{MFH}$

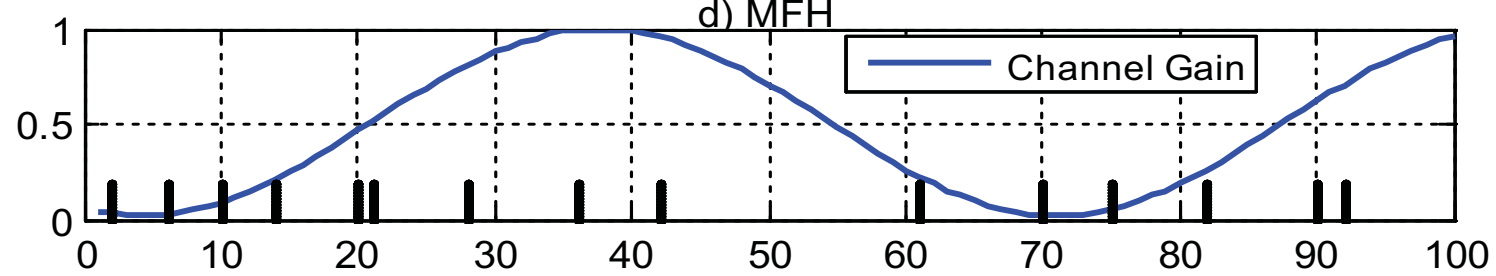

e) $\mathrm{RFH}$

Figure 3.2: Illustrative example of the hopping sets selected by the different schemes.

as the selected sub-bands become more adjacent, it becomes easier for the jammer to jam a large number of them within a limited bandwidth. Furthermore, HGFH tends to select sub-bands that are very close to each other, and the separation increases from $\mathrm{AFH}$ to $\mathrm{CMFH}$ to $\mathrm{MFH}$. As the separation increases, the performance of the communication system becomes less effected by the presence of adaptive PBJ. It is also worth noting that although RFH has good performance in the presence of 


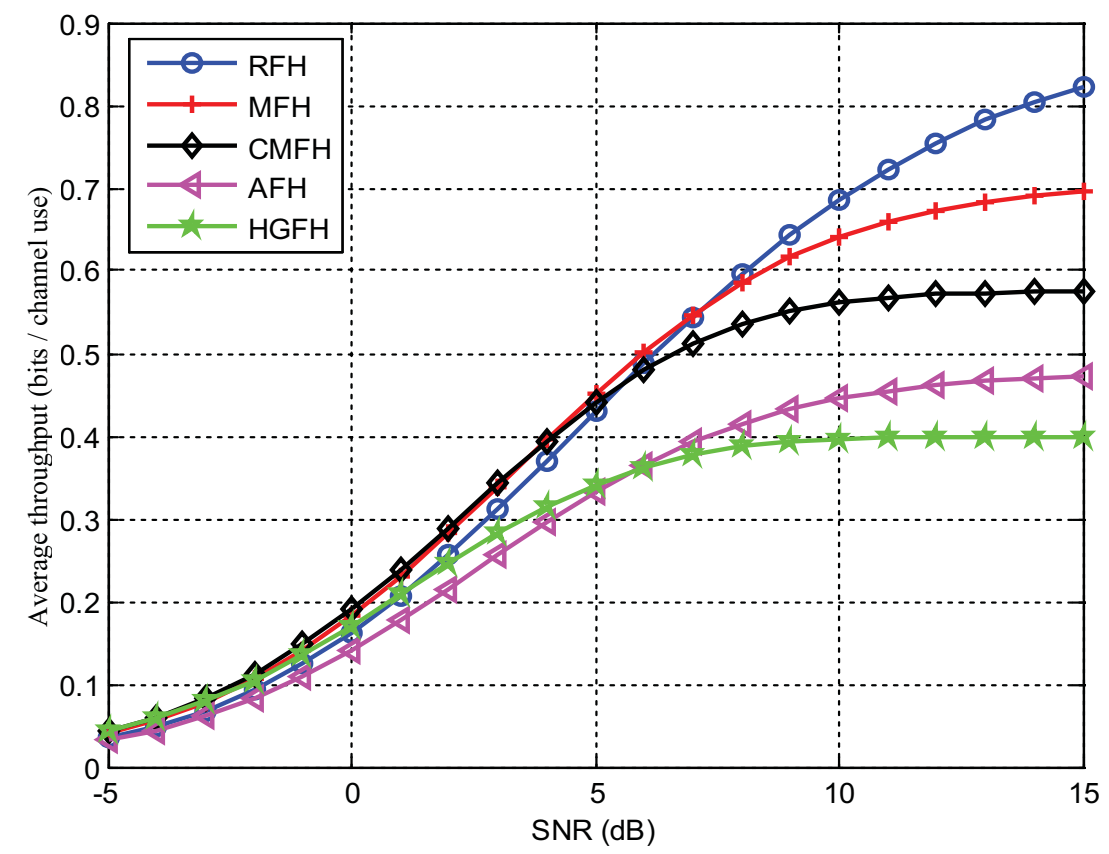

Figure 3.3: Average throughput of the hopping schemes in the presence of adaptive jamming.

adaptive PBJ at high SNRs, because it is channel ignorant it suffers from selecting sub-bands with low gains, degrading its performance at low SNRs.

\subsection{Parametric Optimization}

In the previous section, we presented and investigated the different frequency hopping schemes in the absence and presence of jamming. When we were investigating the performance of $\mathrm{MFH}, \mathrm{CMFH}$ and $\mathrm{AFH}$ schemes, we were assuming predetermined values for the number of selected sub-bands, $M$, the threshold value, $T$, and the regulation value, $\alpha$. In the following section we are interested in improving the performance of these frequency hopping schemes by optimizing the values of the parameters $M, T$ and $\alpha$. 


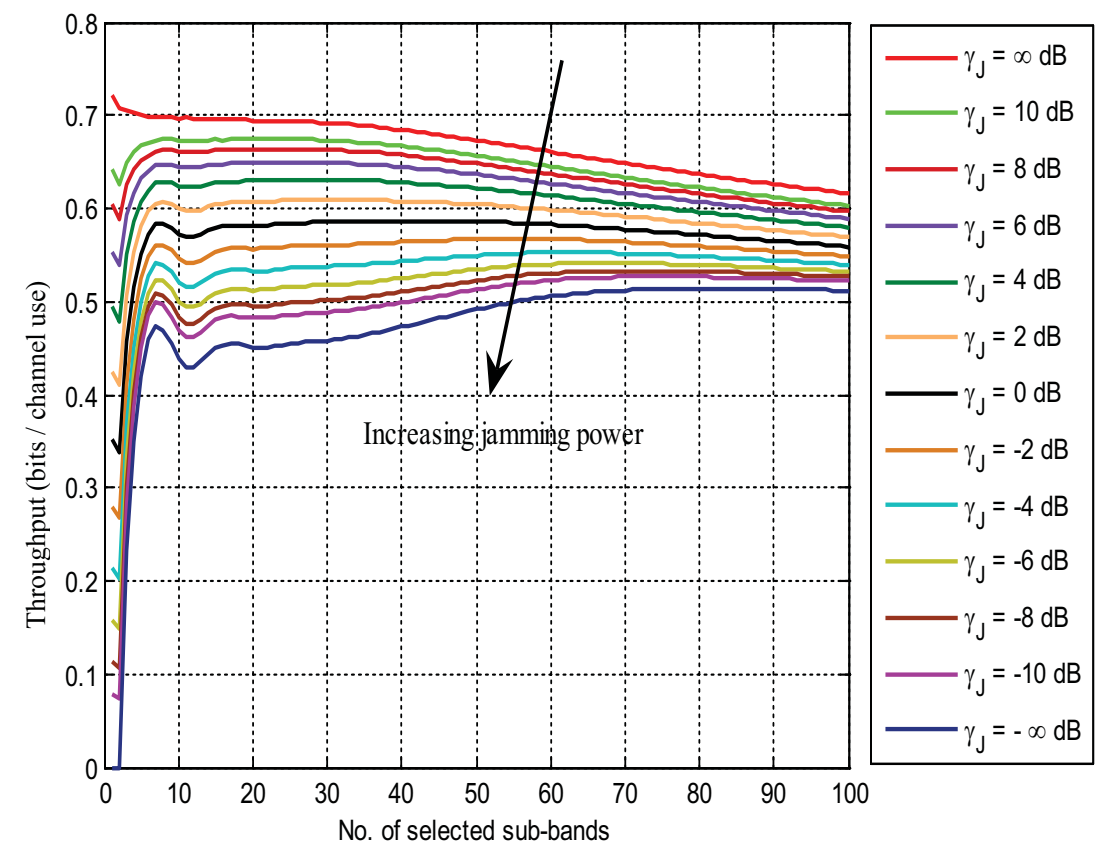

Figure 3.4: The throughput vs. the size of the hopping set for $\mathrm{MFH}$ with different jamming powers and $5 \mathrm{~dB}$ SNR.

\subsubsection{Optimized Matched Frequency Hopping (OMFH)}

In the original MFH scheme, the author proposed selecting $10-15 \%$ of the available sub-bands for the hopping set. We propose to optimize the throughput performance of $\mathrm{MFH}$ by optimizing the only parameter that we have, which is the number of selected sub-bands, $M$.

Fig. 3.4 shows the average throughput of the MFH scheme as a function of the size of the hopping set, $M$, at a fixed SNR of $5 \mathrm{~dB}$, for a range of jamming powers. The jamming power range from -10 to $10 \mathrm{~dB}$, and the extremes of no jamming $\left(\gamma_{J}=+\infty\right.$ $\mathrm{dB})$ and infinite power jamming $\left(\gamma_{J}=-\infty \mathrm{dB}\right)$ are also included. Careful study of this figure yields some useful insight into the behavior of the system. When $M=1$, the hopping set contains only one sub-band, which will quite likely have a high channel gain. However, this sub-band will certainly be jammed, so the throughput drops 
significantly as the jamming power increases. When $M$ is increased to two, both subbands will be jammed (recall that the PBJ that we are using is able to jam two disjoint frequency bands, so the jammer is free to adapt to jam both selected sub-bands). Furthermore, when two sub-bands are selected, their channel gains are less likely to be the best (and certainly they cannot both be the best), so the average throughput drops, regardless of the jamming power. When $M$ is increased to 3 , the jammer is probably unable to jam all three sub-bands, so the throughput improves dramatically, particularly when the jamming power is strong. This phenomenon continues with increasing $M$ until when $M$ is about 7 . At this point the dispersion of the subbands in the hopping set becomes small enough that the jammer is able to jam more than two sub-bands, so the performance starts to drop. As $M$ increases past about 11, the fraction of sub-bands that are jammed starts to decrease, and the jammer becomes less likely to jam the best sub-bands, so the performance starts to increase. This continues until the hopping set gets too large, so a large number of severely attenuated sub-bands are included in the hopping set, causing the throughput to once again diminish. Note that this general behavior is more pronounced when the jamming is strong. When the jamming is weak the throughput mostly just decreases as more sub-bands are included, because the effects of fading dominate the effect of jamming. Similar results hold at lower and stronger signal strengths as shown in Figs. 3.5 and 3.6, for SNRs of $0 \mathrm{~dB}$ and $10 \mathrm{~dB}$, respectively.

From the previous discussion it is apparent that there is an optimal value for the size of the hopping set, which varies depending on the SNR and SJR. The optimum hopping set size as a function of the SJR and SNR is shown in Fig. 3.7, and the corresponding optimum throughput is shown in Fig. 3.8. We can see that as the jamming power increases, we need to increase the number of sub-bands to obtain 


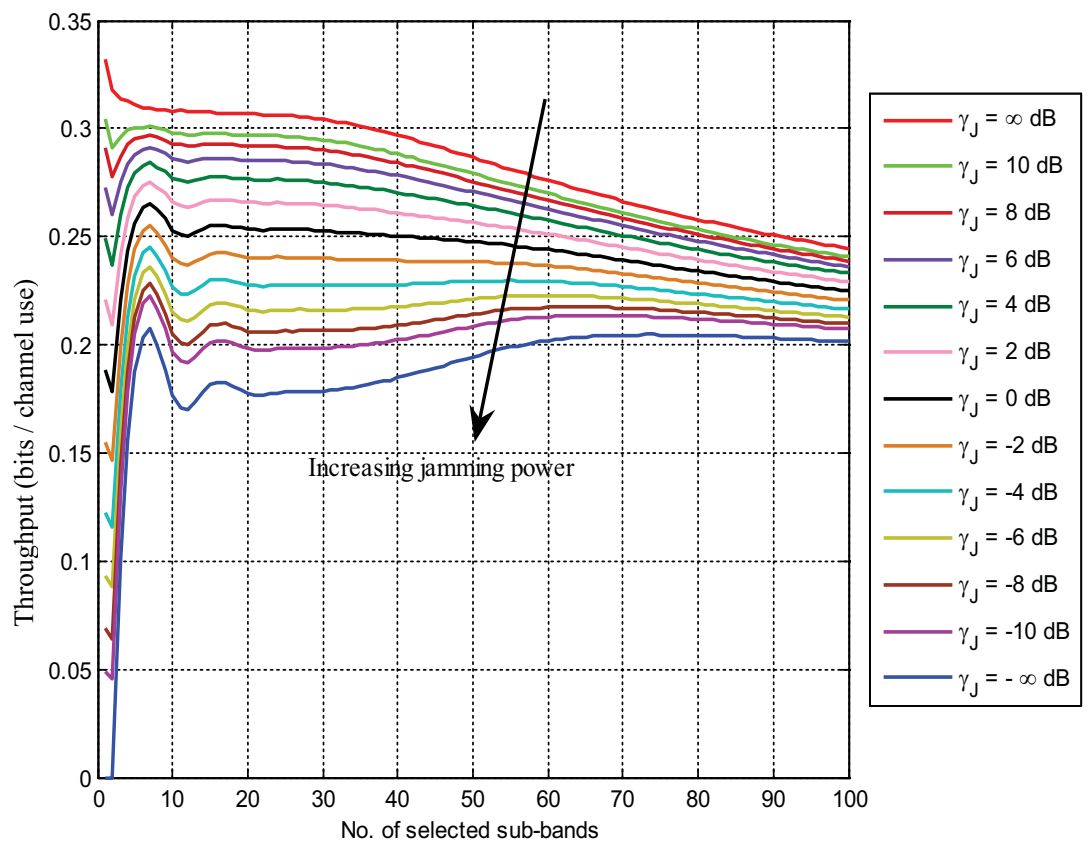

Figure 3.5: The throughput vs. the size of the hopping set for $\mathrm{MFH}$ with different jamming powers and $0 \mathrm{~dB}$ SNR.

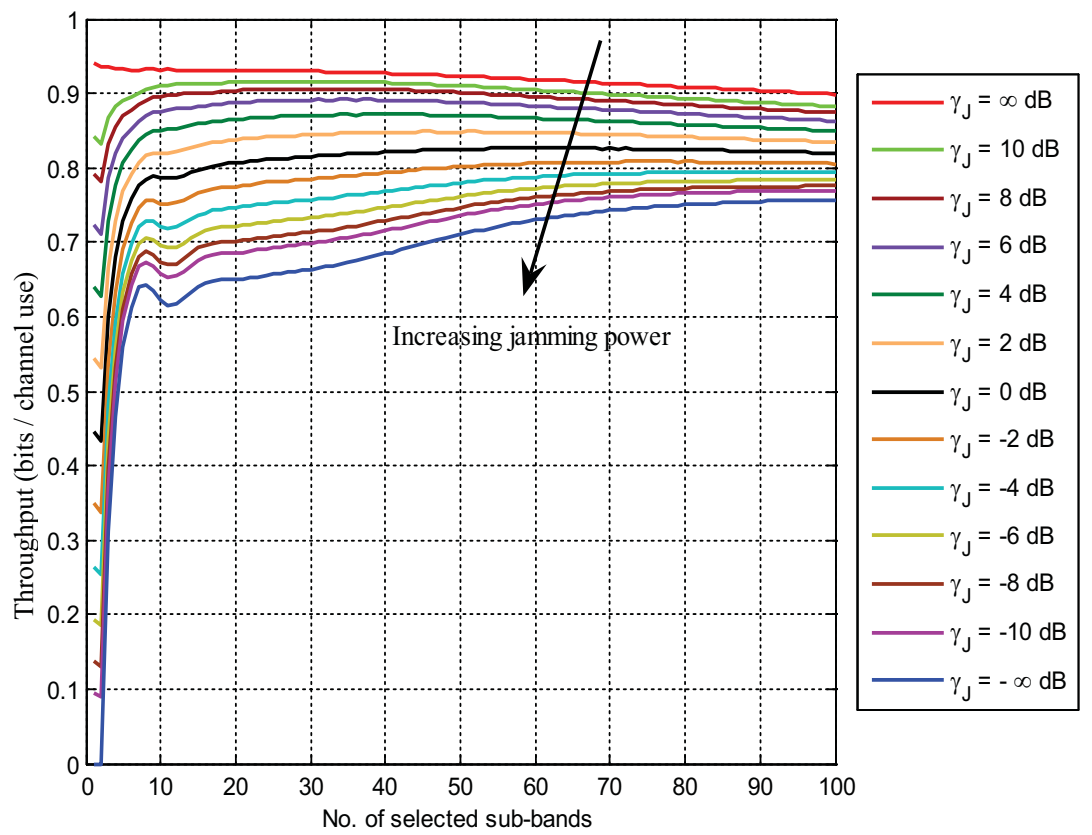

Figure 3.6: The throughput vs. the size of the hopping set for $\mathrm{MFH}$ with different jamming powers and $10 \mathrm{~dB}$ SNR. 
higher throughput. However, by using the optimal hopping set size, the throughput only degrades slightly as the jamming power increases.

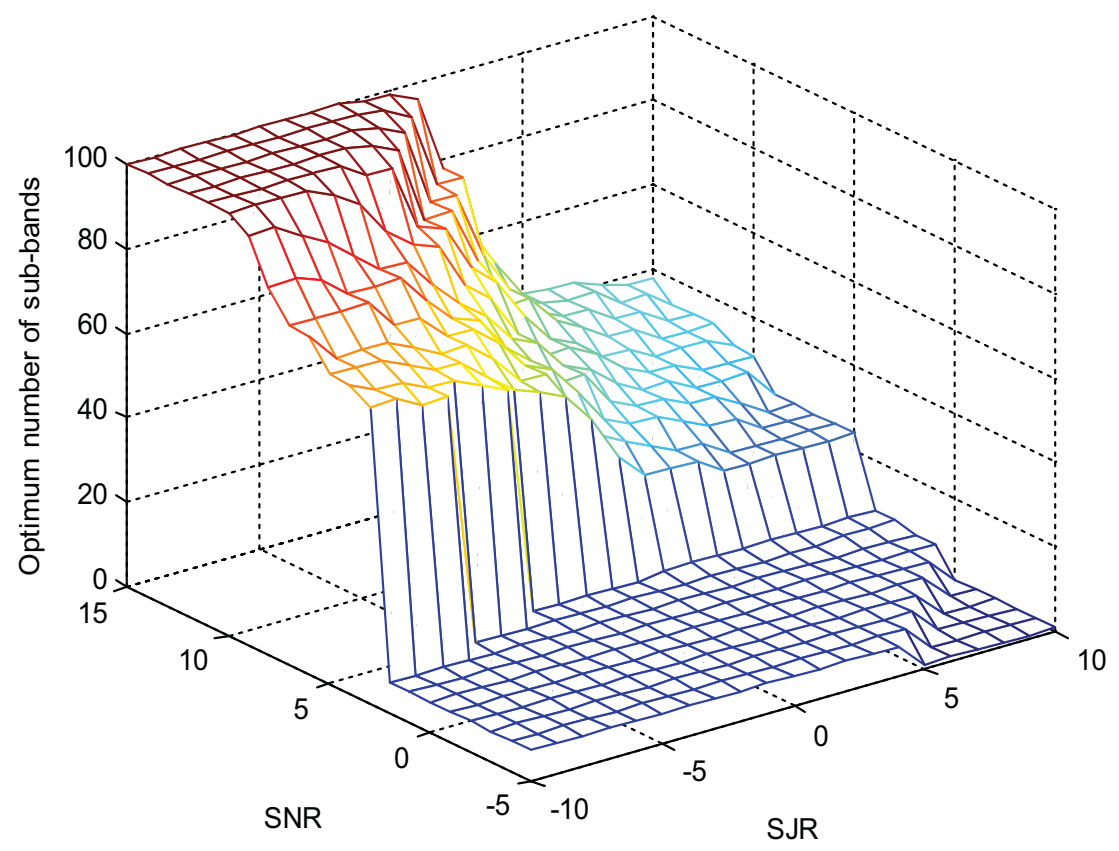

Figure 3.7: The optimum hopping set size for MFH with the presence of different jamming power and SNR scenarios.

It is worth noting that the rather "jagged" nature of the curve in Fig. 3.7 is a result of statistical uncertainly. Even though the results were averaged over 10,000 different channel realizations, which was enough to estimate the throughput with sufficient accuracy (the throughput changed by much less than $1 \%$ compared to when only 1000 channel realizations were used), because the throughput curves are fairly flat in Figs. 3.4 - 3.6, small, statistically insignificant variations in the throughput can cause widely different values in the optimal number of sub-bands.

We compared the throughput performance of the original MFH scheme and the optimized MFH (OMFH) scheme when the $\mathrm{SNR}=0,5$ and $10 \mathrm{~dB}$ as shown in Figs. 3.9 to 3.11. The results show that OMFH is always better than MFH, and the 


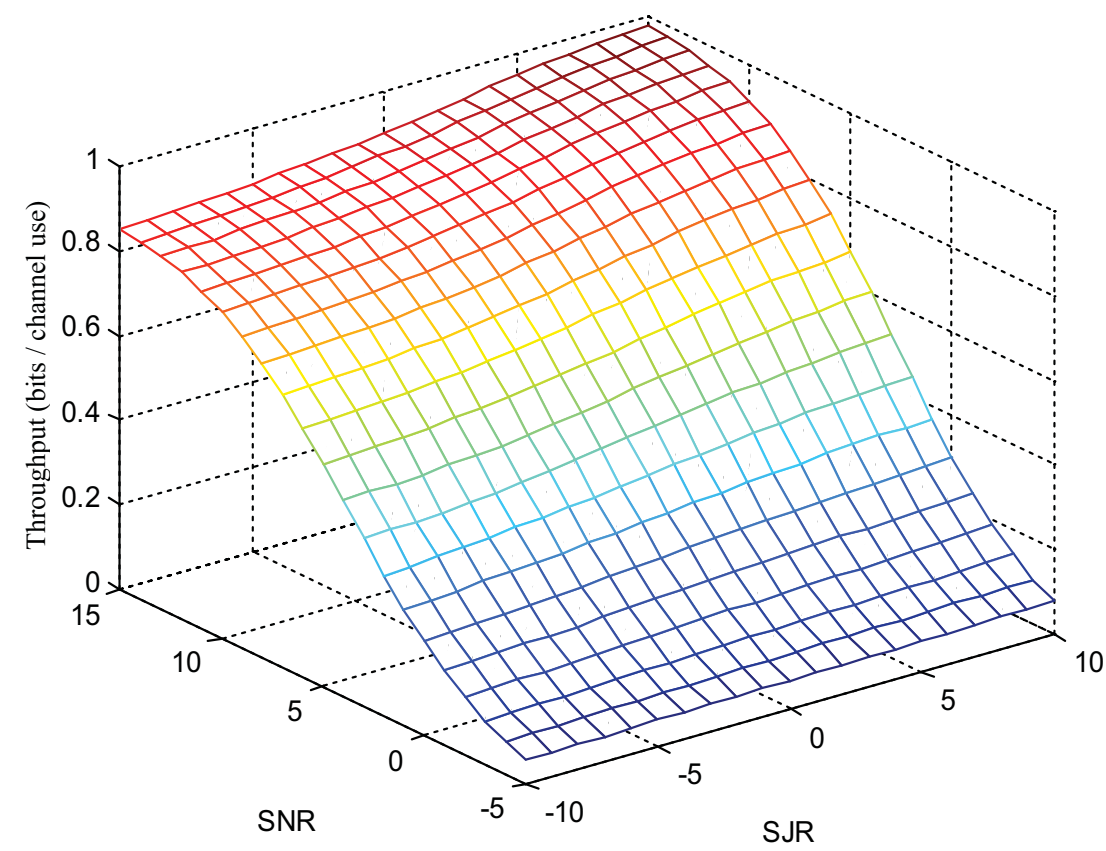

Figure 3.8: The optimum throughput of MFH for different jamming power and SNR scenarios.

advantage is more pronounced when the jamming is strong. For example, when the $\mathrm{SNR}=10 \mathrm{~dB}$ and the $\mathrm{SJR}=-10 \mathrm{~dB}$ there is a $14 \%$ improvement in the throughput.

\subsubsection{Optimized Clipped Matched Frequency Hopping (OCMFH)}

In $\mathrm{CMFH}$ there are two main predetermined control parameters: the size of the hopping set, $M$, and the threshold value, $T$. In [72] these two parameters were previously assumed to be 15 and 0.2 respectively. The question is, do these parameters give the best throughput performance? For that reason we investigated the throughput performance when $M=15$ for $T$ in the range of $[0,1]$ for different jamming power scenarios and with $\mathrm{SNR}=5 \mathrm{~dB}$ as shown in Fig. 3.12. The results show that although assuming $M=15$ sub-bands and $T=0.2$ does not give the best throughput, it does achieve good performance in the presence of strong jamming. However, in the presence of weak jamming letting $T=0.2$ is not a good choice, and the best 


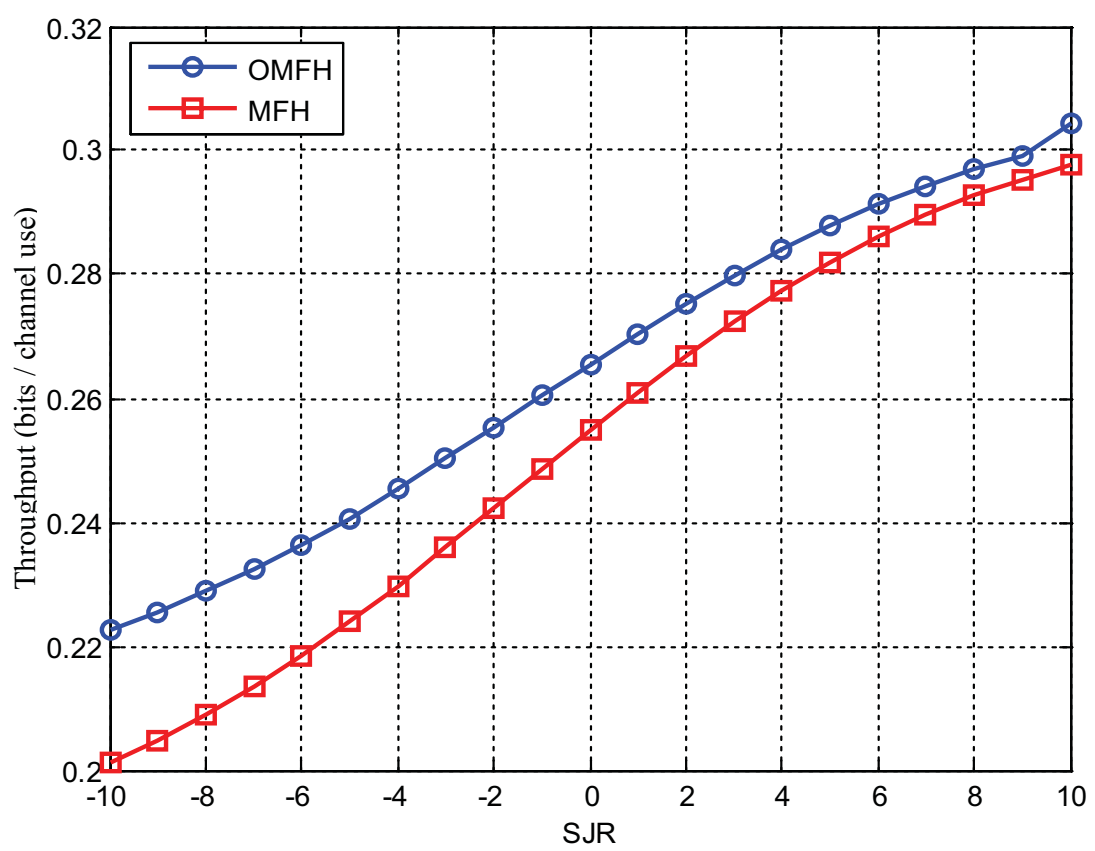

Figure 3.9: Throughput comparison of $\mathrm{MFH}$ and $\mathrm{OMFH}$ when the $\mathrm{SNR}=0 \mathrm{~dB}$.

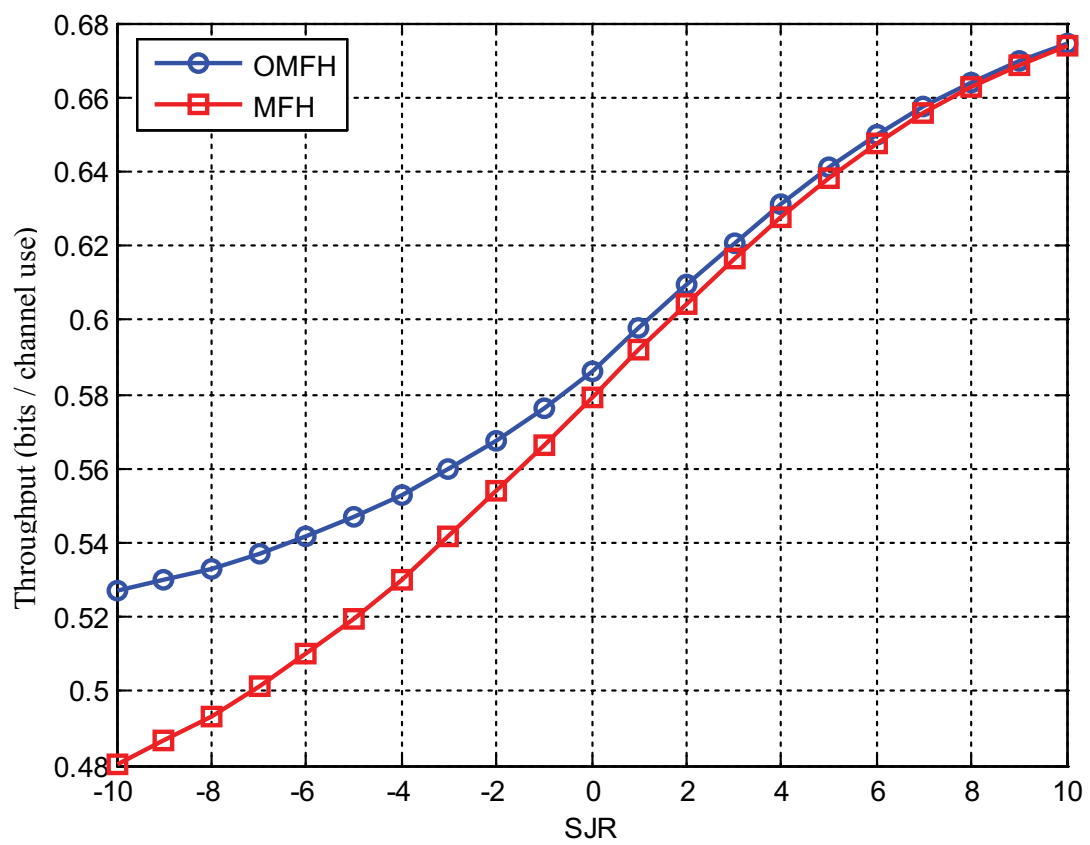

Figure 3.10: Throughput comparison of $\mathrm{MFH}$ and $\mathrm{OMFH}$ when the $\mathrm{SNR}=5 \mathrm{~dB}$.

throughput is obtained when $T=1$. For large value of $T$, the channel gains are clipped to zero for all but the best sub-band in $\mathrm{CMFH}$, so the cumulative metric 


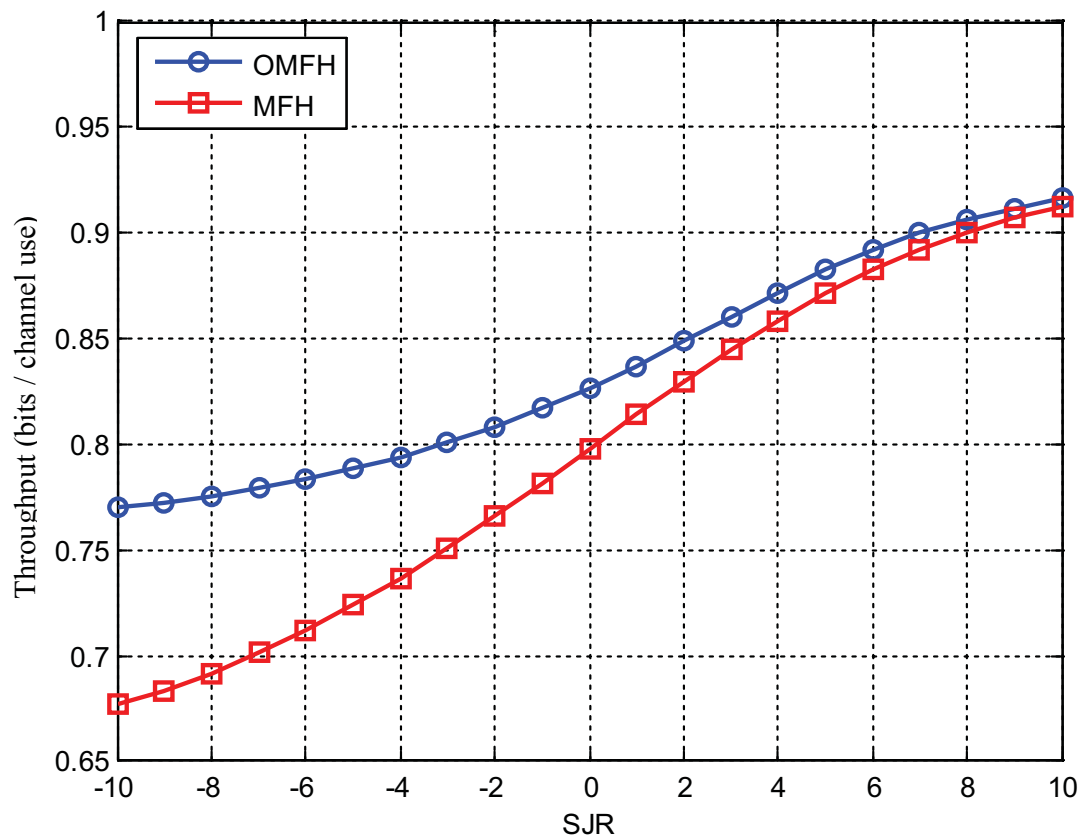

Figure 3.11: Throughput comparison of $\mathrm{MFH}$ and $\mathrm{OMFH}$ when the $\mathrm{SNR}=10 \mathrm{~dB}$.

becomes a step function, with the step occurring at the index of the best sub-band. This means that although the algorithm attempts to select $M=15$ sub-bands, only one unique sub-band (the best sub-band) is in fact selected. This is very beneficial when the jamming is weak (i.e. $\gamma_{J} \geq 4 \mathrm{~dB}$ ).

We also investigated the throughput performance for $\mathrm{CMFH}$ when $T=0.2$ at each value of $M \in\{1,2, \ldots, K\}$ for different jamming power scenarios and with SNR $=5 \mathrm{~dB}$ as shown in Fig. 3.13. The results show that when $T=0.2$ selecting the hopping set size $M=15$ is not the best choice. For that reason the optimization for CMFH should include the combination of the two parameters, $M$ and $T$, to get the optimum throughput.

To find the optimum throughput performance of CMFH we calculated the average throughput over 10,000 different channel realizations using Monte Carlo simulation, 


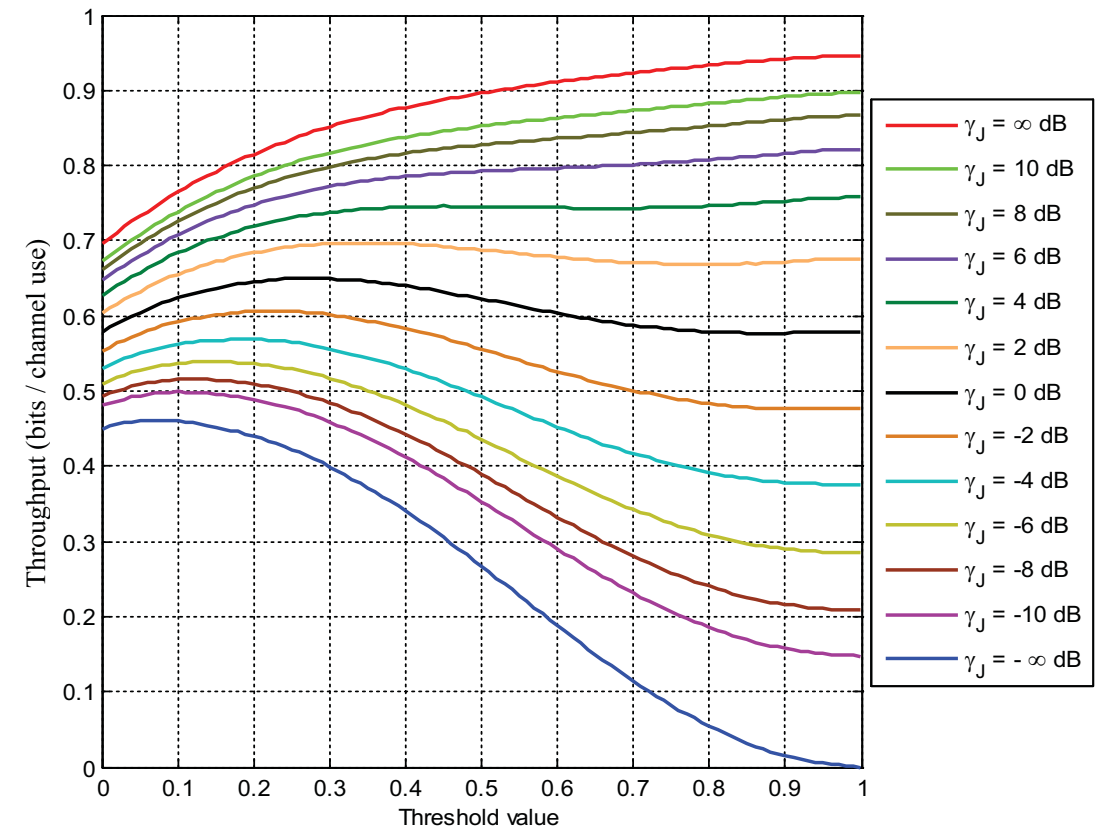

Figure 3.12: The throughput performance of CMFH vs. $T$ when $M=15$ and $\mathrm{SNR}=$ $5 \mathrm{~dB}$ for different jamming power scenarios.

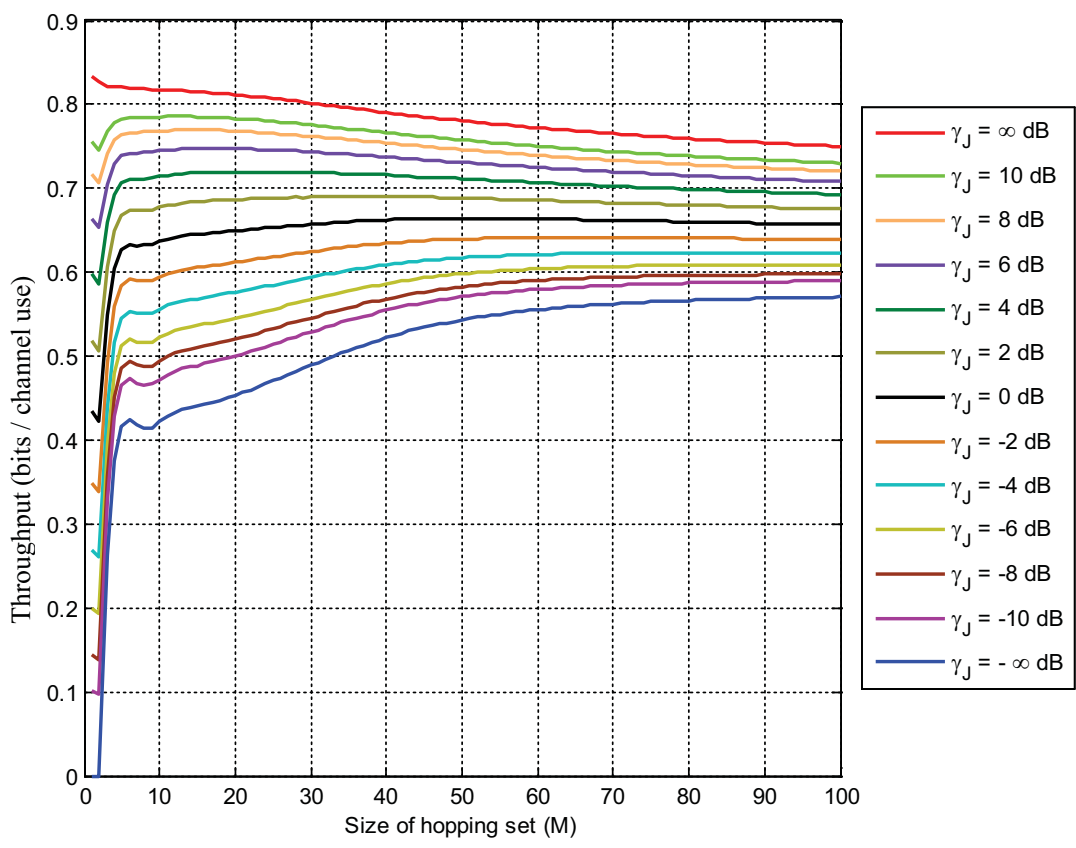

Figure 3.13: The throughput performance of CMFH vs. $M$ when $T=0.2$ and $\mathrm{SNR}=$ $5 \mathrm{~dB}$ for different jamming power scenarios. 


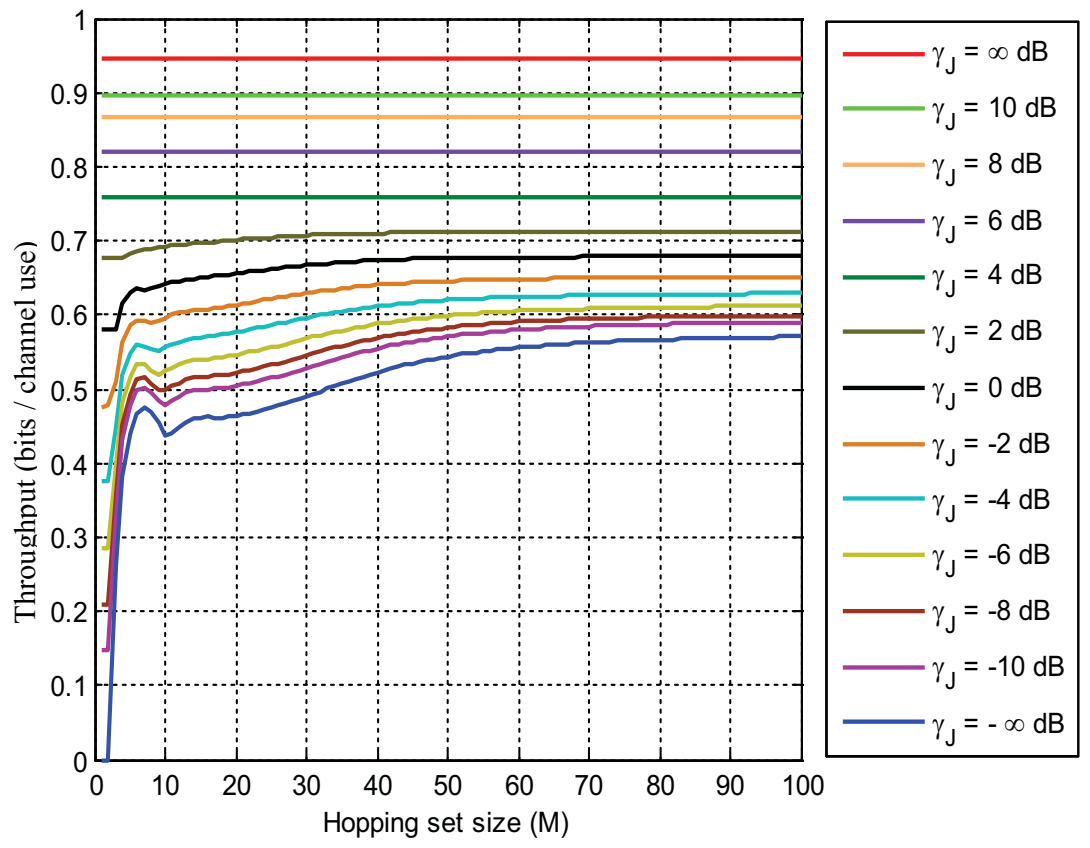

Figure 3.14: The throughput vs. the size of the hopping set for CMFH with different jamming powers and $5 \mathrm{~dB}$ SNR. The optimum threshold is used for each $M$.

for all the available values of $M$ and $T$. We then selected the hopping set size $M$, and the threshold value, $T$, that achieve the maximum average throughput for different jamming power scenarios and SNR values.

The optimum throughput for CMFH by optimizing $T$ at each value of $M$ when the $\mathrm{SNR}=5 \mathrm{~dB}$ is shown in Fig. 3.14. We can see that with weak jamming, the throughput performance of OCMFH does not change much when the number of subbands, $M$, is increased, and as the jammer gets stronger the throughput is highest when $M=100$. It is worth remembering that using a large value for $M$ does not necessarily mean that a large number of sub-bands will be selected, as the number of actually selected sub-bands also depends on $T$. This is illustrated in Fig. 3.15, which shows the actual number of selected sub-bands vs. $M$, for different values of $T$. Clearly when $T$ is large only a small number of sub-bands is selected, regardless of 


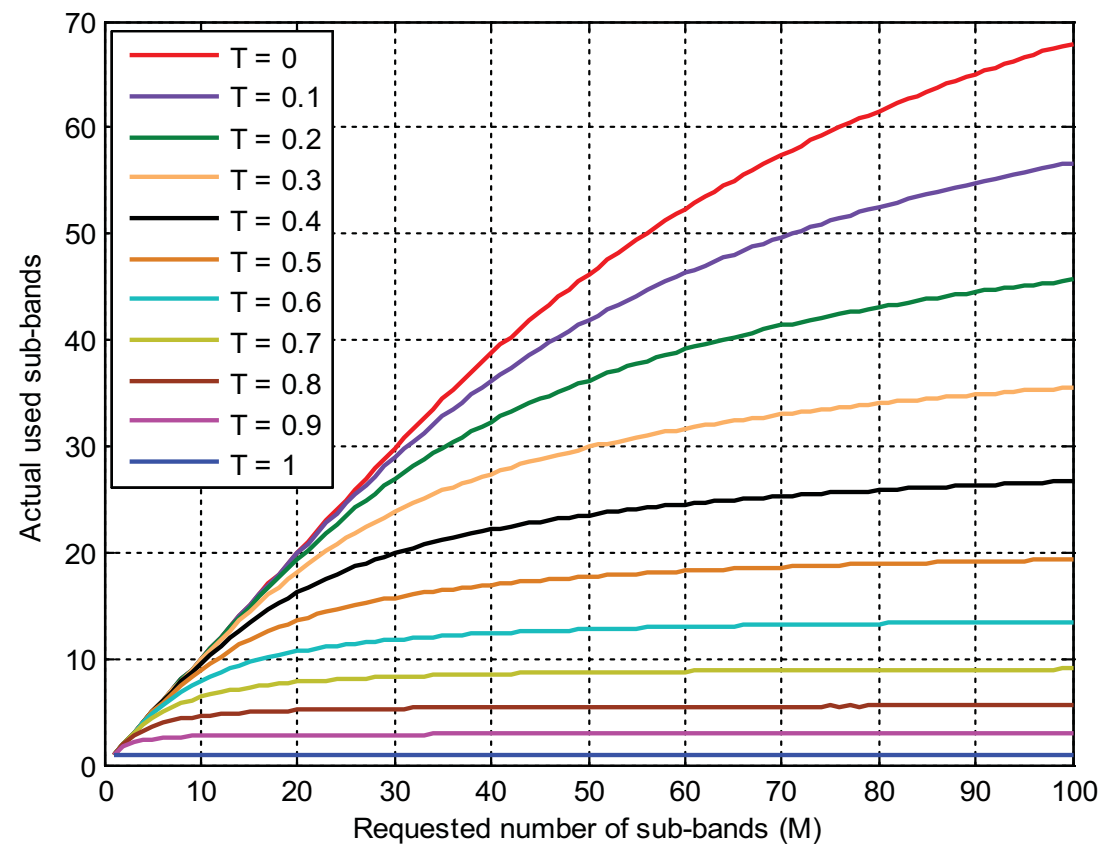

Figure 3.15: The requested number of sub-bands vs. the actual number of used subbands.

$M$. And even when $T$ is smaller the number of actually selected sub-bands is usually less than $M$.

We also investigated the throughput performance when optimizing the size of the hopping set, $M$, at each value of the threshold level $T$ at $\mathrm{SNR}=5 \mathrm{~dB}$ as shown in Fig. 3.16. The results show that when the jamming power is strong the value of $T=$ 0.2 provides good throughput performance when we optimize $M$. In weak jamming it is better to select $T=1$ to obtain the optimum throughput performance. We can also notice that the throughput and optimizing $M$ is better than assuming $M=15$, when $T=0.2$. To obtain better throughput we need to decrease the threshold level when the jamming power increases because that allows the CMFH scheme to select a larger number of sub-bands to mitigate the effect of the strong jammer. Of course by selecting a large number of sub-bands the system will suffer from the channel fading, but its effect will be less than the effect of strong jammer. 


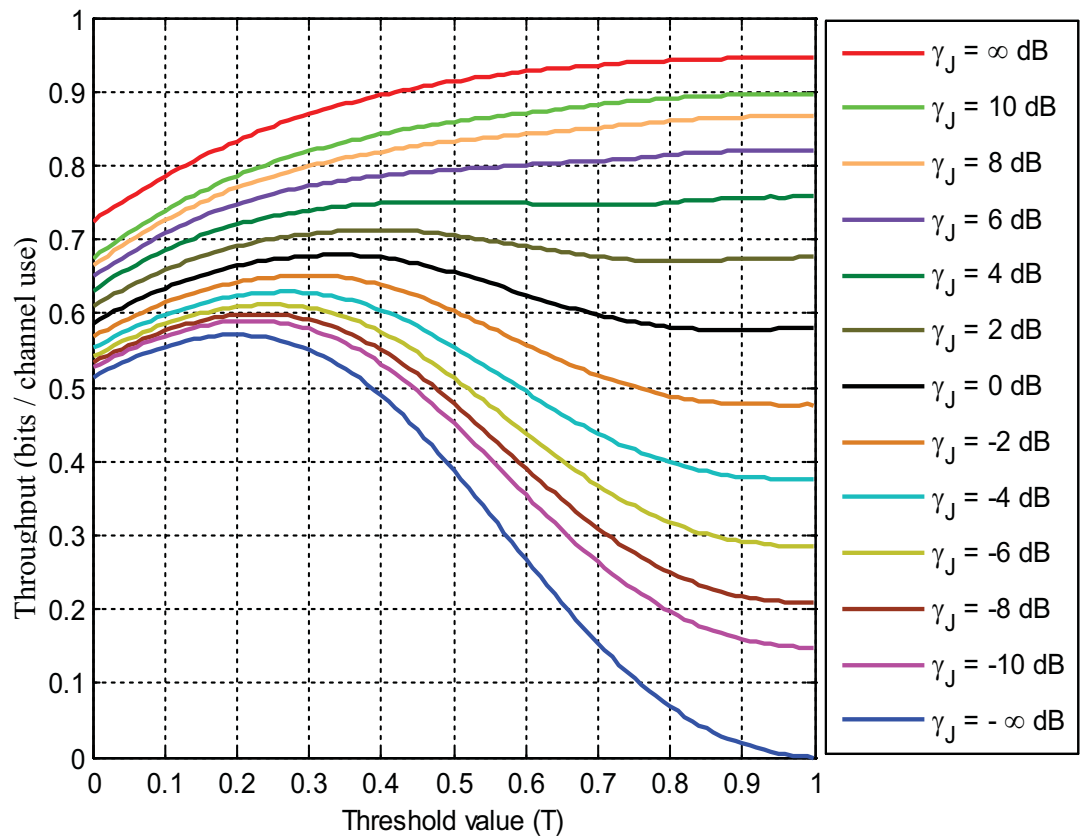

Figure 3.16: The throughput vs. the threshold value for CMFH with different jamming powers and $5 \mathrm{~dB}$ SNR. The optimal hopping set size is used for each $T$.

The optimum threshold value in the presence of different jamming power and different SNR values is shown in Fig. 3.17. From Fig. 3.17 we can see that CMFH is better than MFH because when we optimize the throughput we found that the minimum value of $T$ is greater than zero. $\mathrm{MFH}$ is a special case of $\mathrm{CMFH}$ when the threshold value, T, equals zero. Fig. 3.18 shows the optimum number of sub-bands for different SNR and SJR values. We see that as the jamming power decreases, it is better to use CMFH to select one sub-band and as the jamming power increases, the number of sub-bands should be increased to get the optimum throughput.

It is clear that we need to adjust the threshold level and the number of sub-bands to obtain the optimum throughput. The optimum throughput is shown in Fig. 3.19 in the presence of different jamming power and SNR values.

We investigated the throughput improvement of OCMFH compared to $\mathrm{CMFH}$ when the $\mathrm{SNR}=0,5$ and $10 \mathrm{~dB}$. As shown in Fig. 3.20 when the $\mathrm{SNR}=0 \mathrm{~dB}$ 


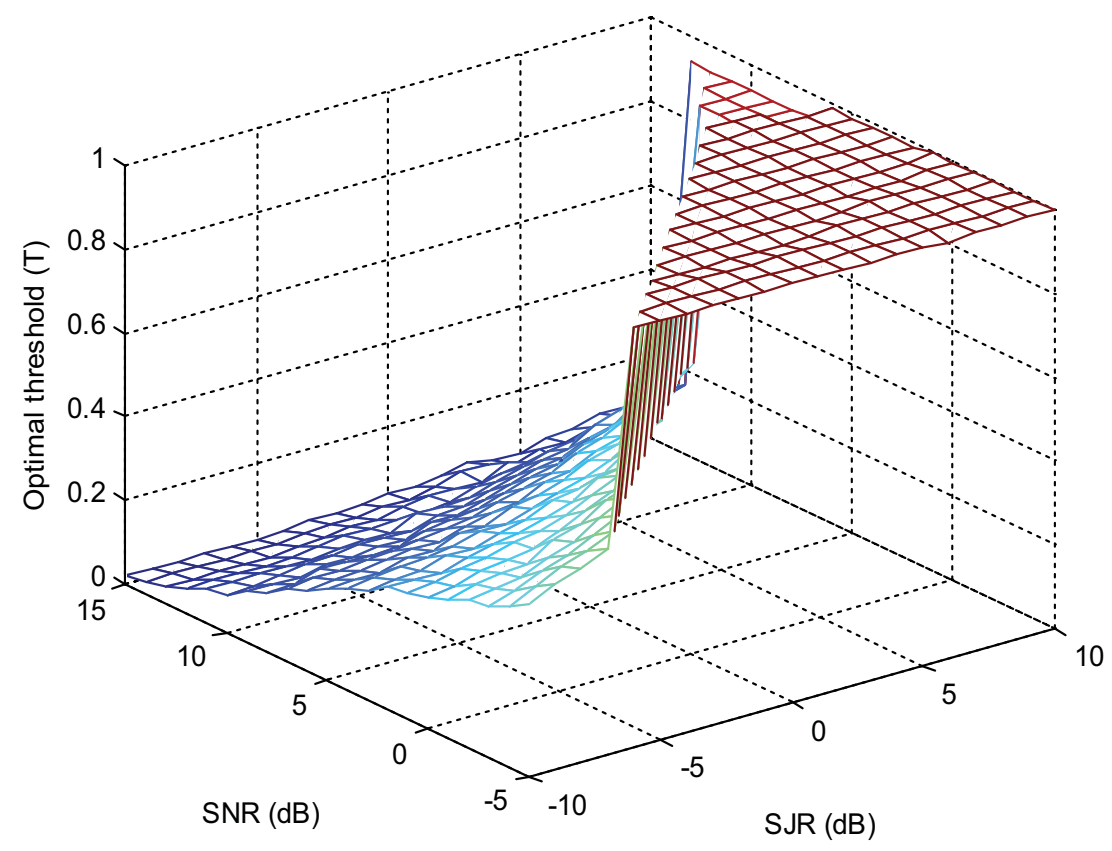

Figure 3.17: The optimum threshold for CMFH with the presence of different jamming power and SNR scenarios.

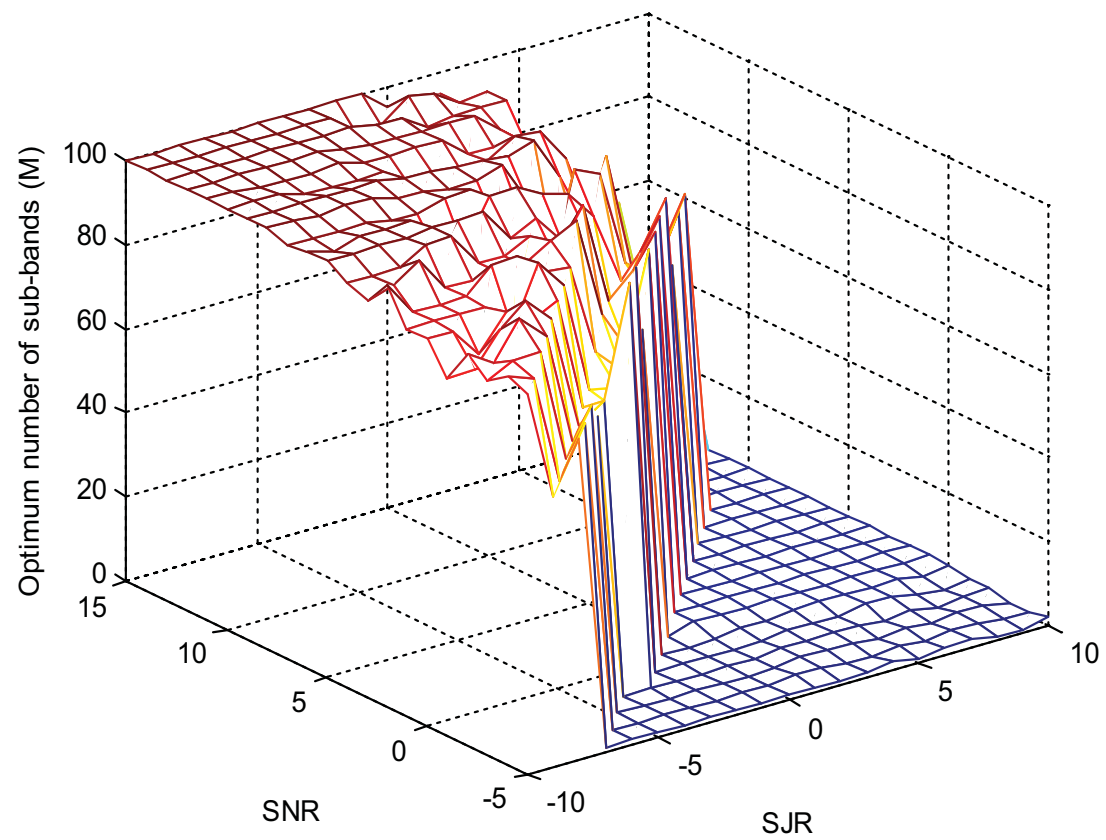

Figure 3.18: The optimum number of sub-bands for CMFH with the presence of different jamming power and SNR scenarios. 


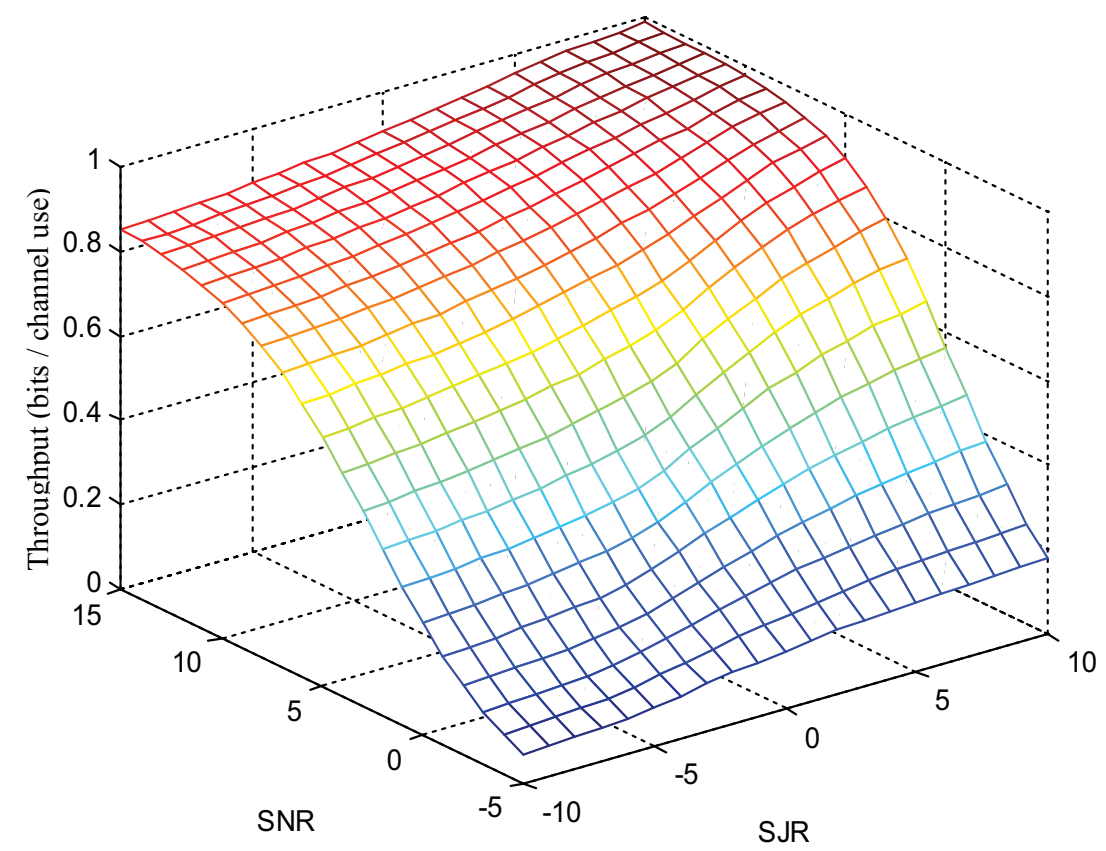

Figure 3.19: The optimum throughput for $\mathrm{CMFH}$ with the presence of different jamming power and SNR scenarios.

there is a $15 \%$ throughput improvement in the presence of strong jamming and a $47 \%$ throughput improvement in the presence of weak jamming. By increasing the SNR to $5 \mathrm{~dB}$, as in Fig. 3.21, we can obtain a $20 \%$ improvement in the presence of strong jamming and $15 \%$ in the presence of weak jamming. As the SNR increases the performance of OCMFH becomes more efficient as we can see in Fig. 3.22 when the $\mathrm{SNR}=10 \mathrm{~dB}$ where the throughput improvement becomes $27 \%$, in the presence of strong jamming.

\subsubsection{Optimized Advanced Frequency Hopping (OAFH)}

In the original AFH scheme [73] $\alpha$ was assumed to be 0.01 and $M=15$. In this section we want to obtain the optimum throughput of AFH by optimizing the regulation value, $\alpha$, and determining the optimum number of sub-bands, $M$, in the presence of adaptive PBJ in frequency selective fading. We will optimize the through- 


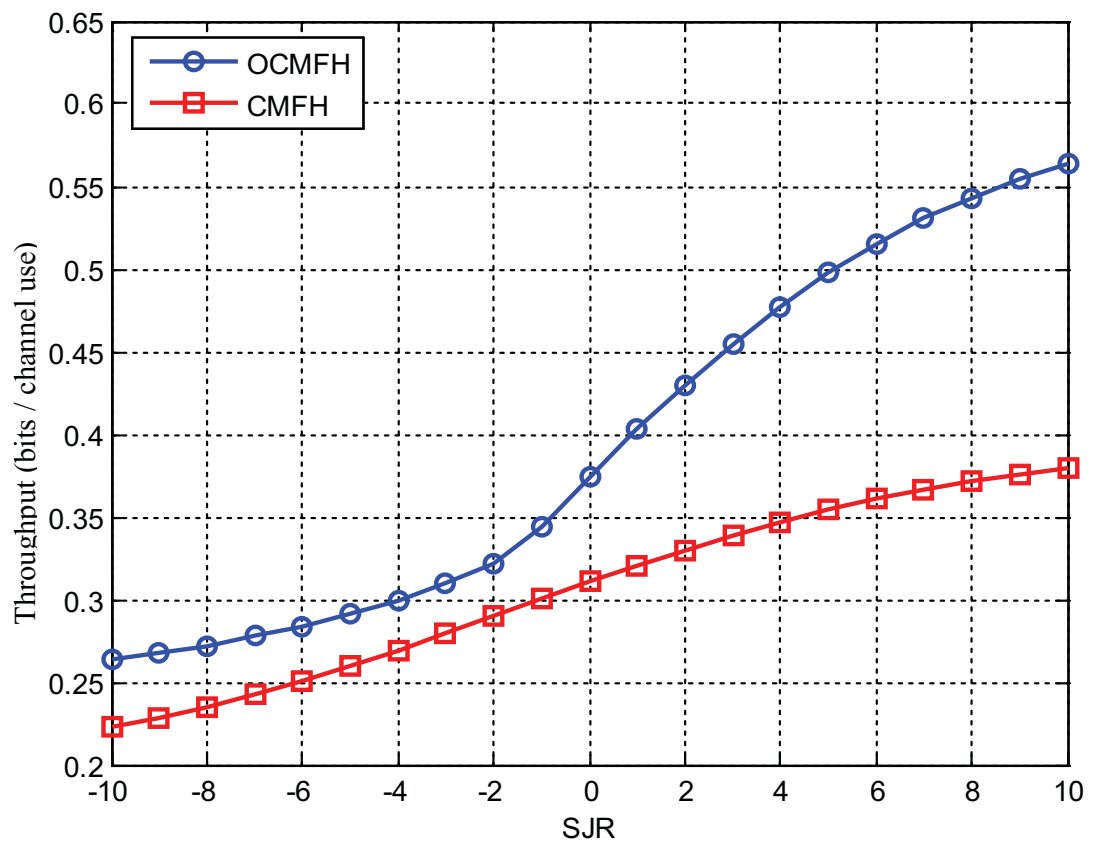

Figure 3.20: Throughput comparison of $\mathrm{CMFH}$ and $\mathrm{OCMFH}$ when the $\mathrm{SNR}=0 \mathrm{~dB}$.

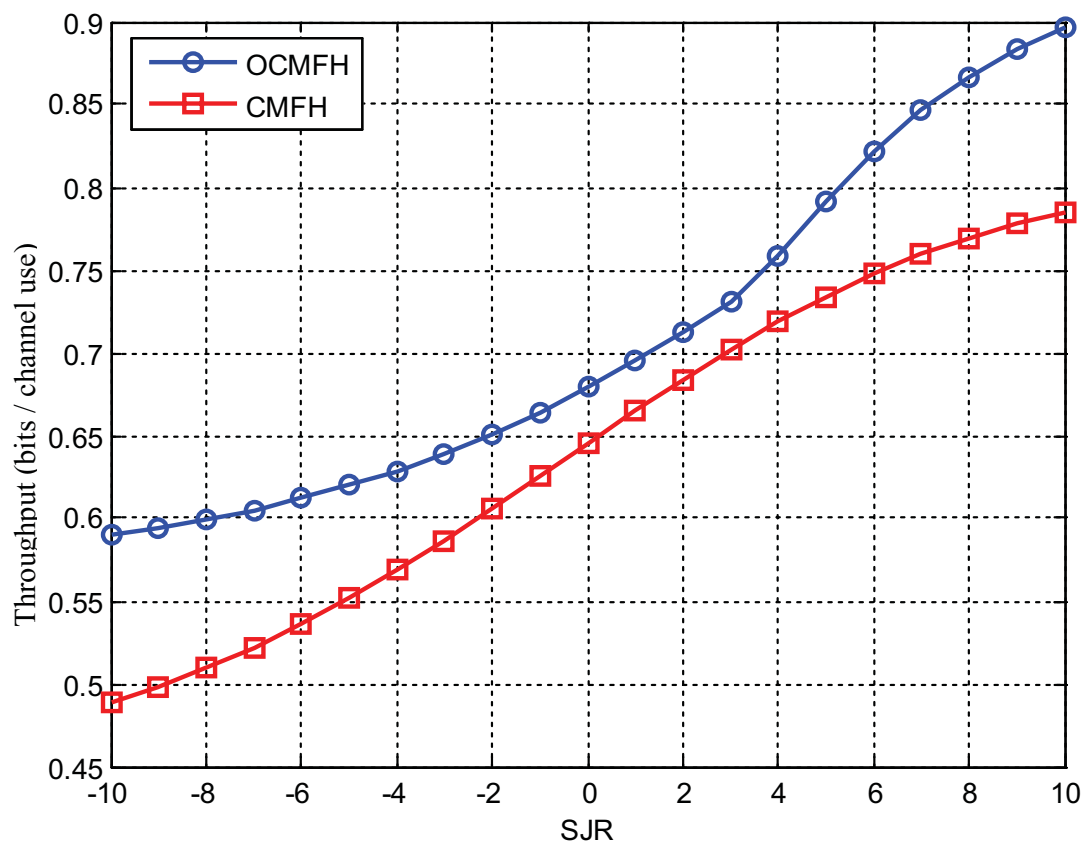

Figure 3.21: Throughput comparison of $\mathrm{CMFH}$ and $\mathrm{OCMFH}$ when the $\mathrm{SNR}=5 \mathrm{~dB}$.

put performance using the same procedures that we used to optimize CMFH.

First, we will investigate the throughput by optimizing $\alpha$ at each value of $M$ 


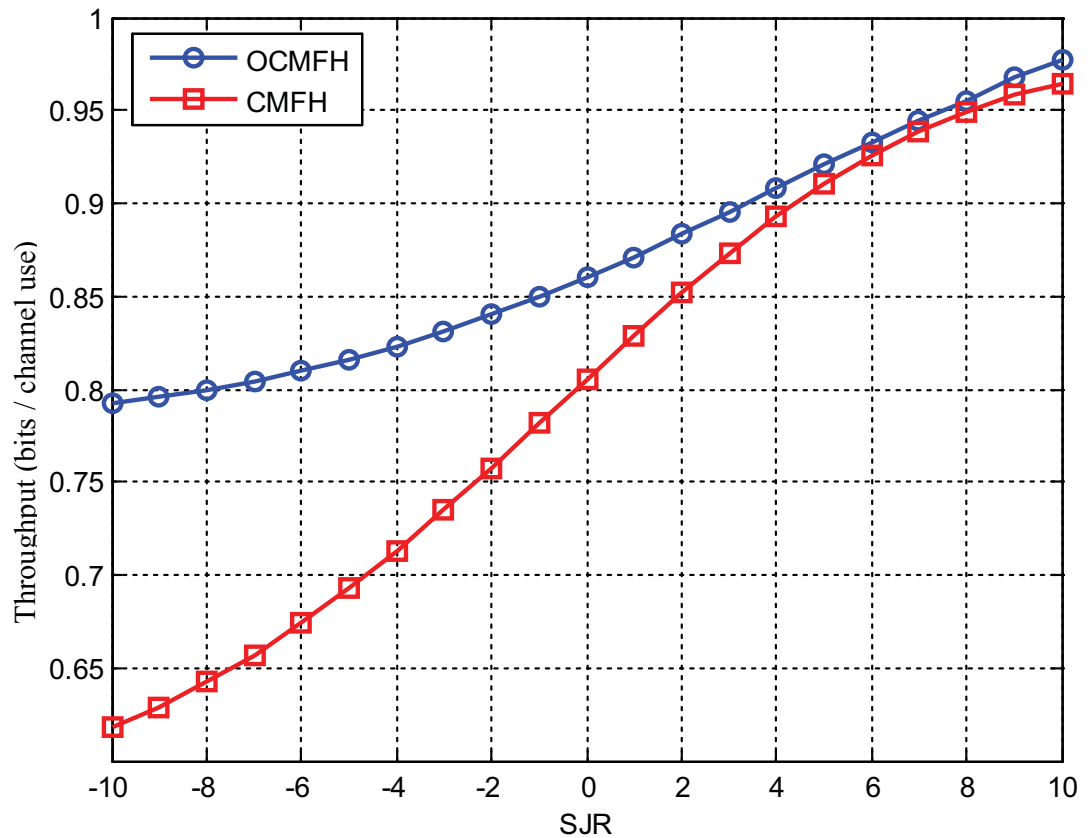

Figure 3.22: Throughput comparison of $\mathrm{CMFH}$ and $\mathrm{OCMFH}$ when the $\mathrm{SNR}=10 \mathrm{~dB}$.

when the $\mathrm{SNR}=5 \mathrm{~dB}$ for different jamming power scenarios as shown in Fig. 3.23. The results show that in the presence of strong jamming it is better to select $M=$ 100 and in the presence of weak jamming it is better to select the best sub-band in the channel (i. e., $M=1$ ).

We also optimized the throughput by optimizing $M$ at different values of $\alpha$ when the $\mathrm{SNR}=5 \mathrm{~dB}$ as shown in Fig. 3.24. The results show that in the presence of strong jamming it is better to select a high value for $\alpha$ to assign a large number of sub-bands to the system and in the presence of weak jamming it is better to use a small value of $\alpha$ to select the sub-band that has the best channel gain.

By using the optimal values of both $M$ and $\alpha$, we can find the optimal throughput, which is shown in Fig. 3.25 for a range of different SNR and SJR values. It is clear the OAFH provides good protection against jamming, regardless of the SNR.

The optimal values for $M$ are shown in Fig. 3.26, where we can see that when 


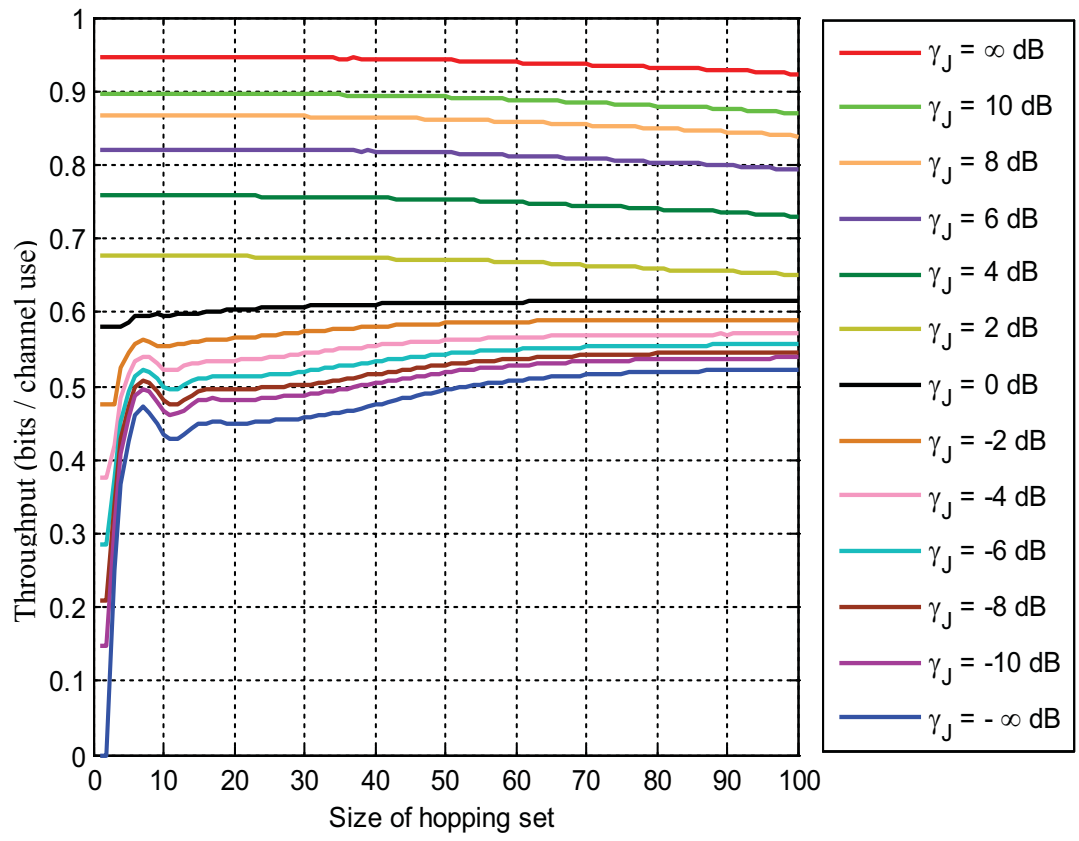

Figure 3.23: The throughput vs. the size of the hopping set for AFH with different jamming powers and $5 \mathrm{~dB}$ SNR.

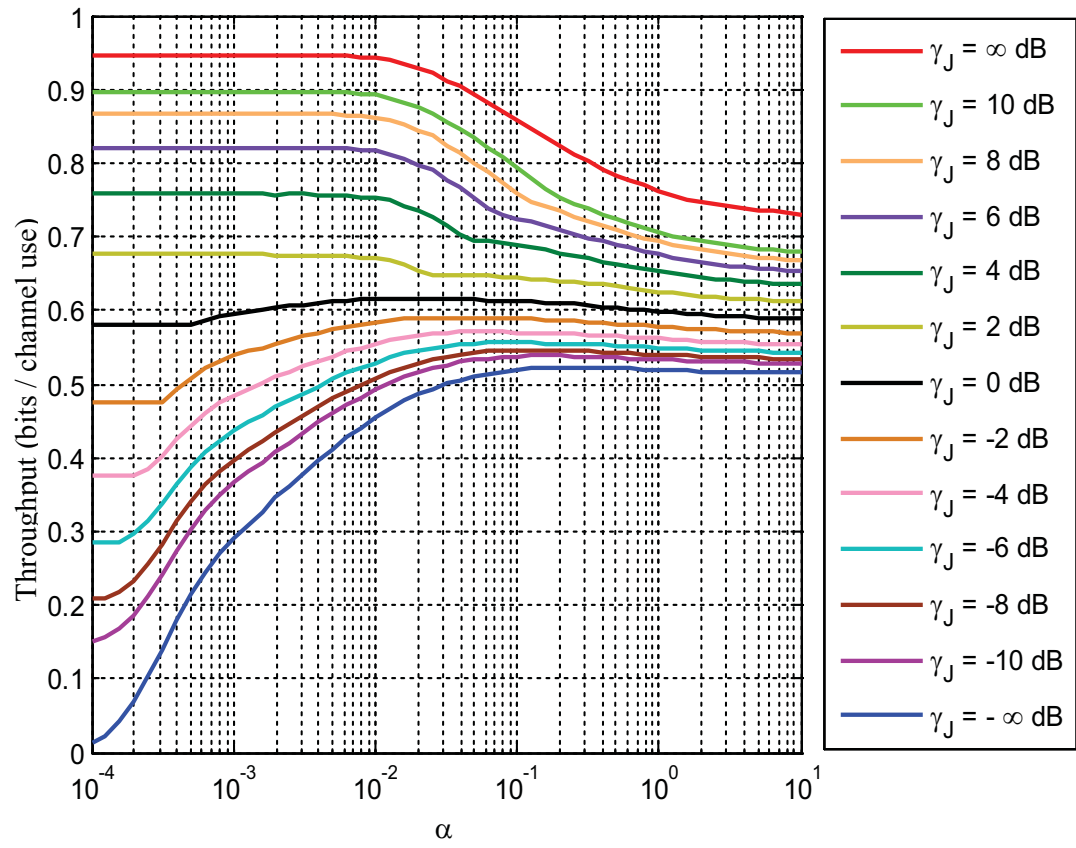

Figure 3.24: The throughput vs. the regulation value for AFH with different jamming powers and $5 \mathrm{~dB}$ SNR. 


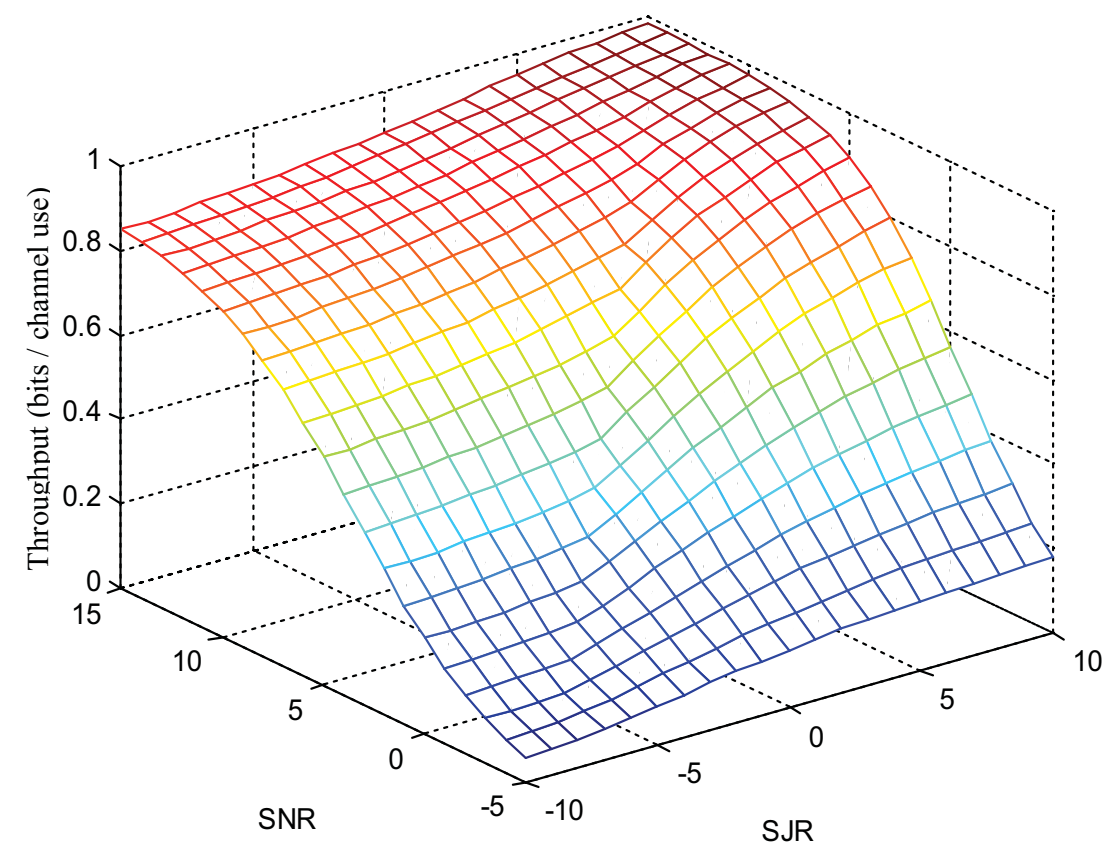

Figure 3.25: The optimum throughput for AFH with the presence of different jamming power and SNR scenarios.

the SNR is large and the SJR is small it is best to use a large value of $M$ to provide better protection against jamming since the fading has a relatively lower impact on performance. Otherwise, a small value of $M$ is preferred. Similarly, as shown in Fig. 3.27, it is better to use a large value of $\alpha$ when the SNR is large and the SJR is small, so many sub-bands are selected. When the SNR is small and the SJR is large, a small value of $\alpha$ forces the system to only select the best sub-band.

The original AFH scheme and the OAFH scheme are also compared to investigate how much throughput improvement we obtained by using OAFH. As shown in Figs. 3.28 to 3.30 when the $\mathrm{SNR}=0 \mathrm{~dB}$ we get $28 \%$ throughput improvement when the jamming power is strong and $33 \%$ improvement when the jamming power is weak. By increasing the SNR the throughput improvement in the presence of strong jamming is getting better as we can see in Fig. 3.29 when the $\mathrm{SNR}=5 \mathrm{~dB}$, the improvement becomes $50 \%$. As the SNR increased to $10 \mathrm{~dB}$ we obtain $70 \%$ throughput 


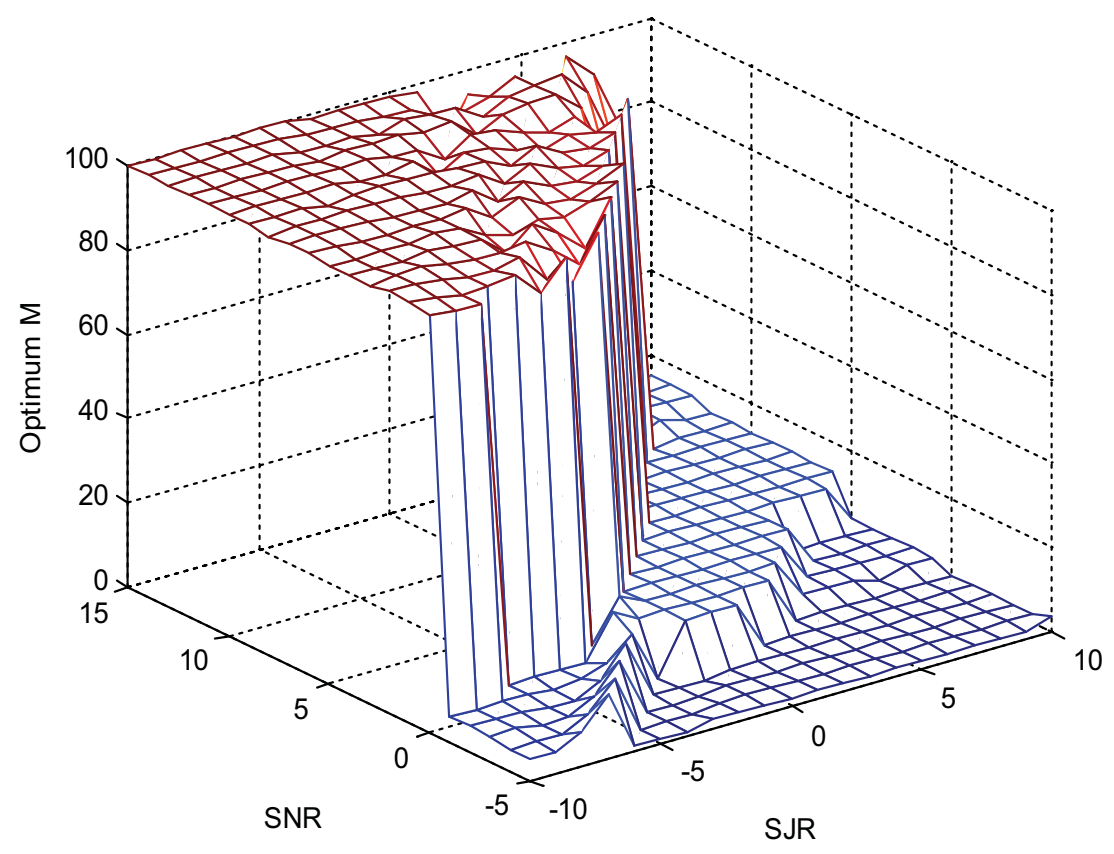

Figure 3.26: The optimum number of sub-bands for OAFH with the presence of different jamming power and SNR scenarios.

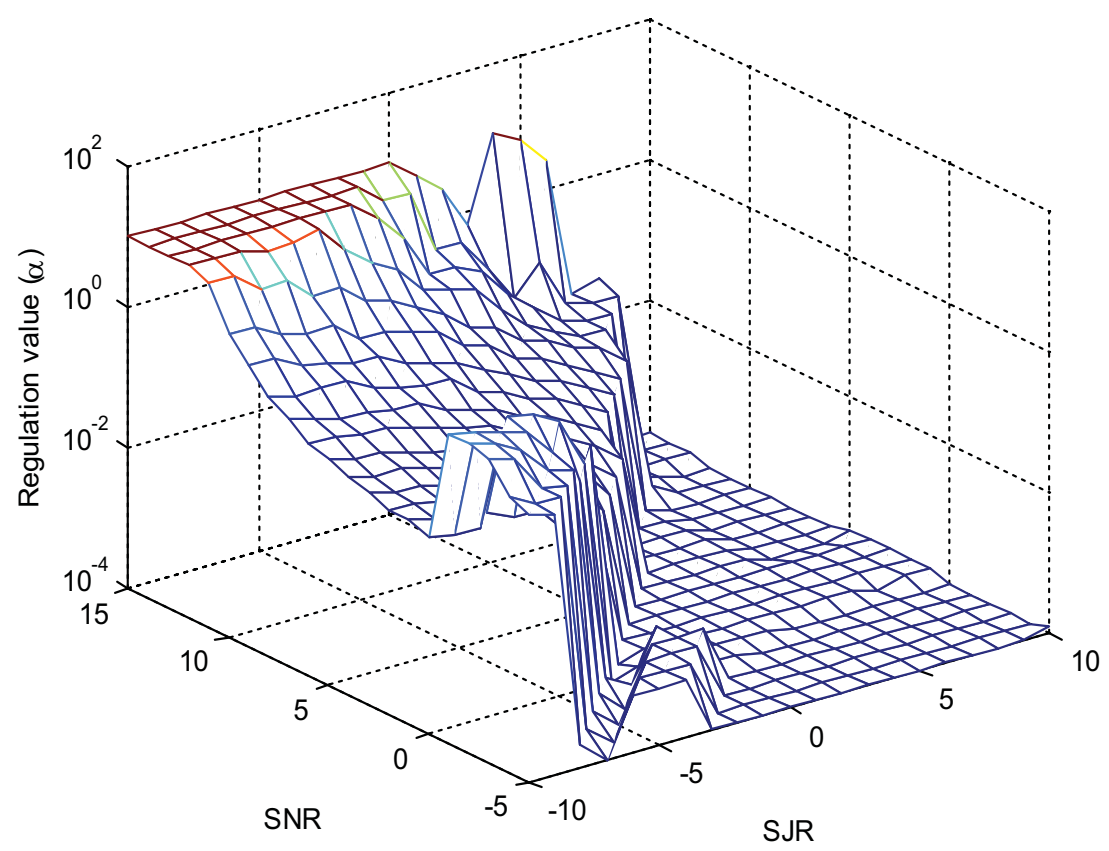

Figure 3.27: The optimum threshold for $\mathrm{OAFH}$ with the presence of different jamming power and SNR scenarios. 


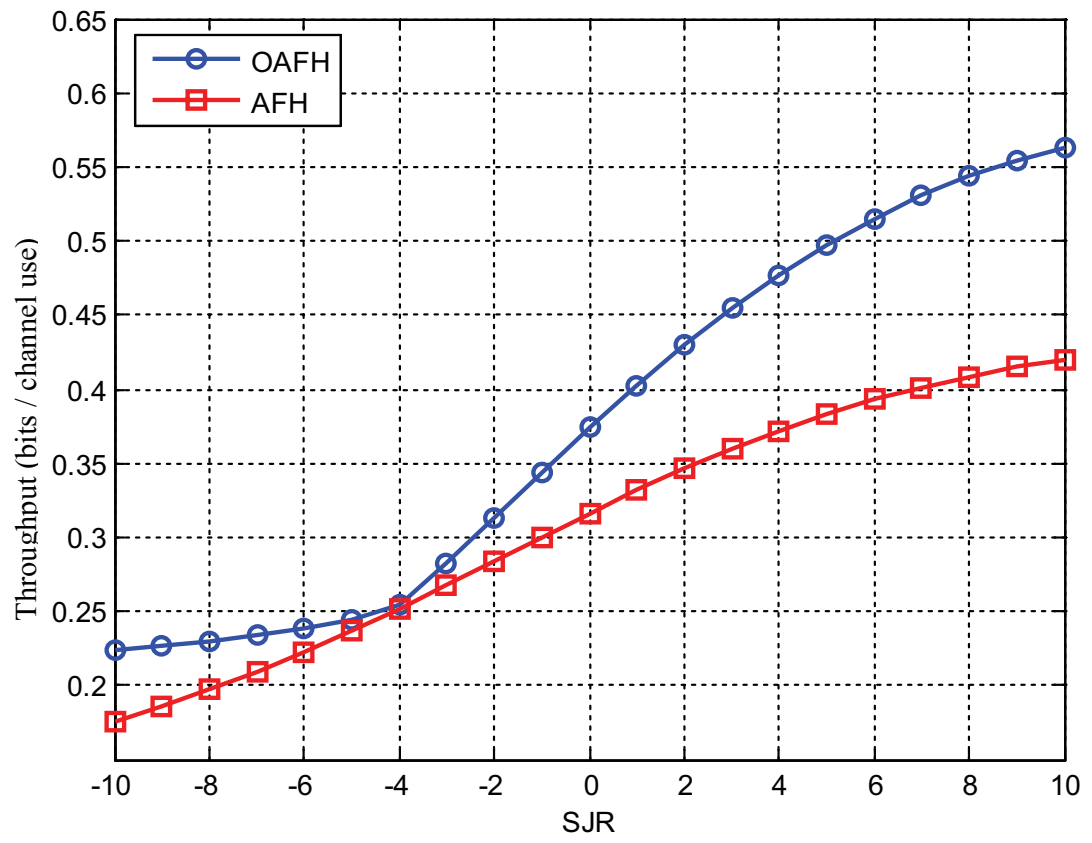

Figure 3.28: Throughput comparison of $\mathrm{AFH}$ and $\mathrm{OAFH}$ when the $\mathrm{SNR}=0 \mathrm{~dB}$.

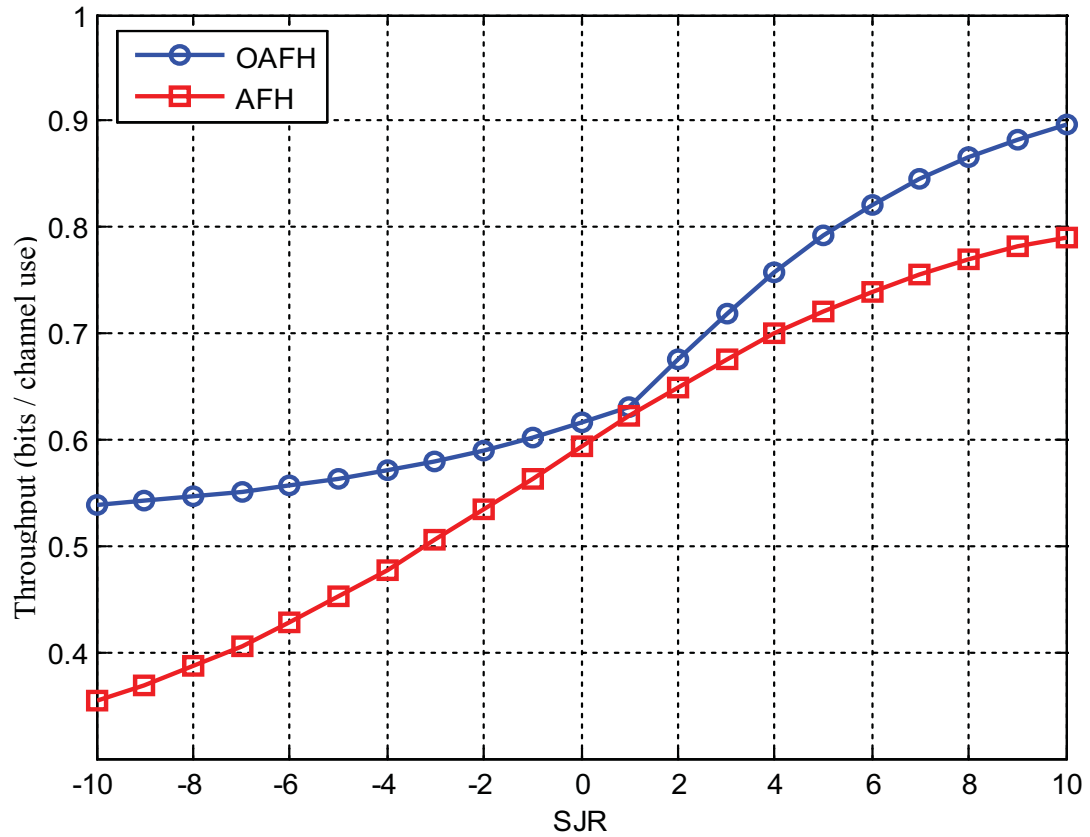

Figure 3.29: Throughput comparison of $\mathrm{AFH}$ and $\mathrm{OAFH}$ when the $\mathrm{SNR}=5 \mathrm{~dB}$.

improvement in the presence of strong jamming. Clearly it is very beneficial to use the optimum values of $M$ and $\alpha$. 


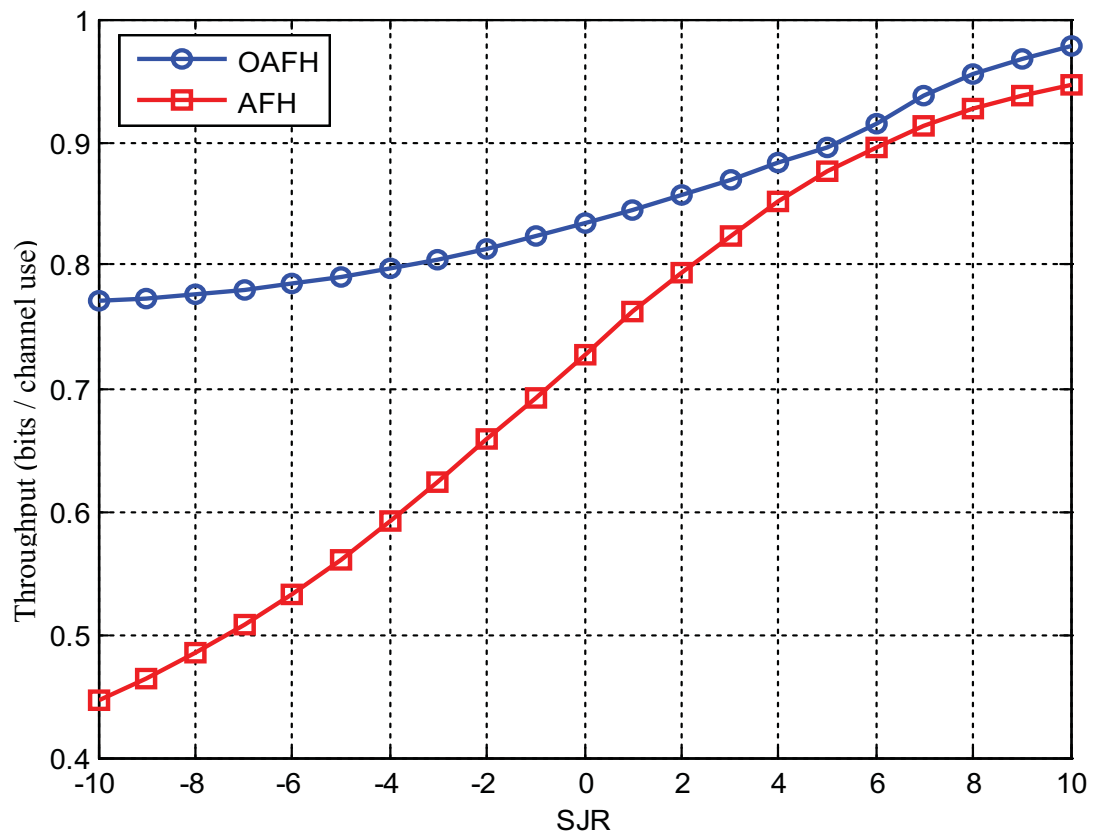

Figure 3.30: Throughput comparison of AFH and $\mathrm{OAFH}$ when the $\mathrm{SNR}=10 \mathrm{~dB}$.

\subsection{Comparisons}

In this section we want to compare the throughput performance of the different optimized frequency hopping schemes in the presence of different jamming power scenarios and different SNR values in frequency selective fading.

To determine which scheme is better to be used in different cases, Fig. 3.31 shows the comparison between the optimized frequency hopping schemes when the $\mathrm{SNR}=5 \mathrm{~dB}$. We can see that OCMFH has the best throughput performance compared to $\mathrm{OAFH}$ and $\mathrm{OMFH}$, and although $\mathrm{OMFH}$ has good throughput performance compared to $\mathrm{MFH}$, it has the worst throughput performance compared to OCMFH and $\mathrm{OAFH}$. We can also notice that $\mathrm{OAFH}$ has the same throughput performance as $\mathrm{OCMFH}$ when the jamming power is weak $(\mathrm{SJR} \geq 4) \mathrm{dB}$ because both schemes select the best sub-band that has the highest channel gain. On the other hand, when the jamming power gets stronger the throughput performance of OAFH becomes closer 


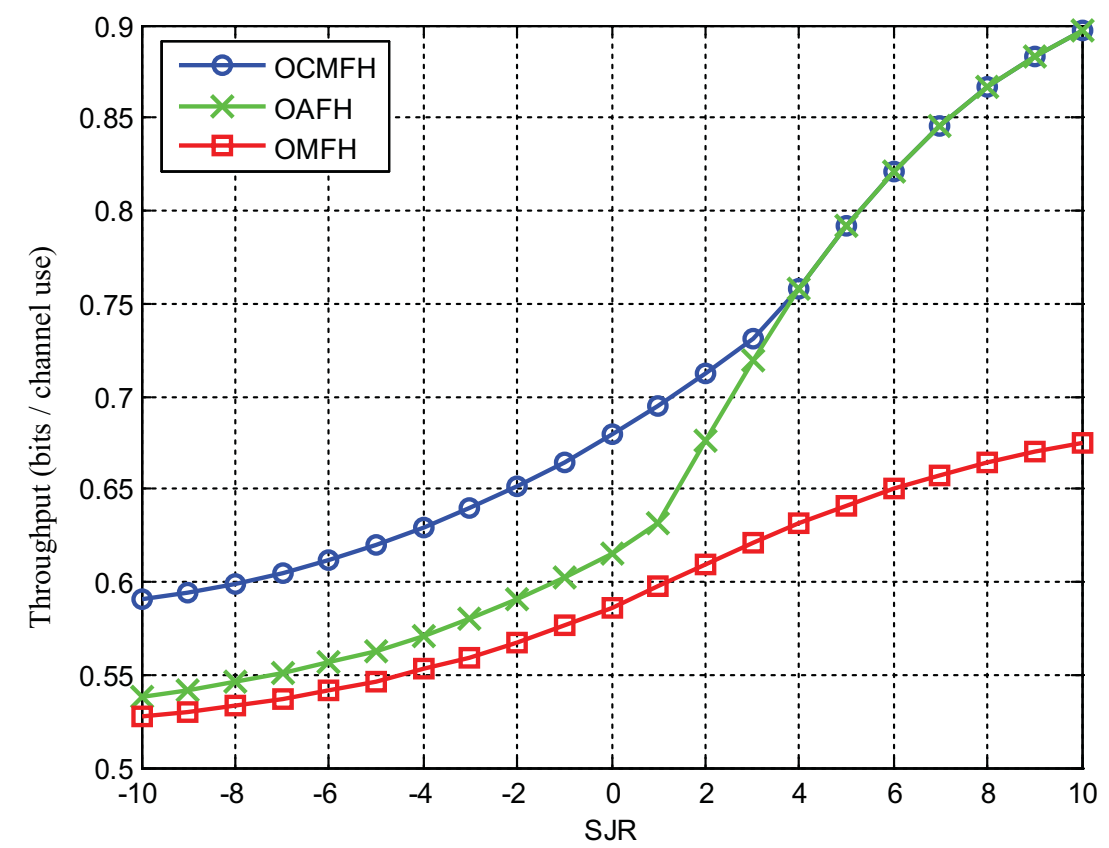

Figure 3.31: Throughput comparison of OMFH, OCMFH and OAFH when the SNR $=5 \mathrm{~dB}$.

to the performance of OMFH. Although both OCMFH and OAFH try to select 100 sub-bands to mitigate the jamming effect, in OCMFH the sub-bands with very small gains have been discarded after the clipping process but in $\mathrm{OAFH}$ with a large value of $\alpha$ these sub-bands still exist and are selected as in OMFH and that degrades its throughput performance.

With lower SNR as shown in Figs. 3.32 and 3.33, it is obvious that OCMFH is still the best scheme and $\mathrm{OAFH}$ is better than $\mathrm{OMFH}$ and its performance approaches the OCMFH when the jamming power getting weaker. We can also notice that with low SNR the performance of OCMFH and OAFH have much better performance than OMFH. When the SNR is high, as shown in Figs. 3.34 and 3.35, the results show that although there is not too much throughput difference between the proposed schemes, OCMFH still has the best throughput performance. 


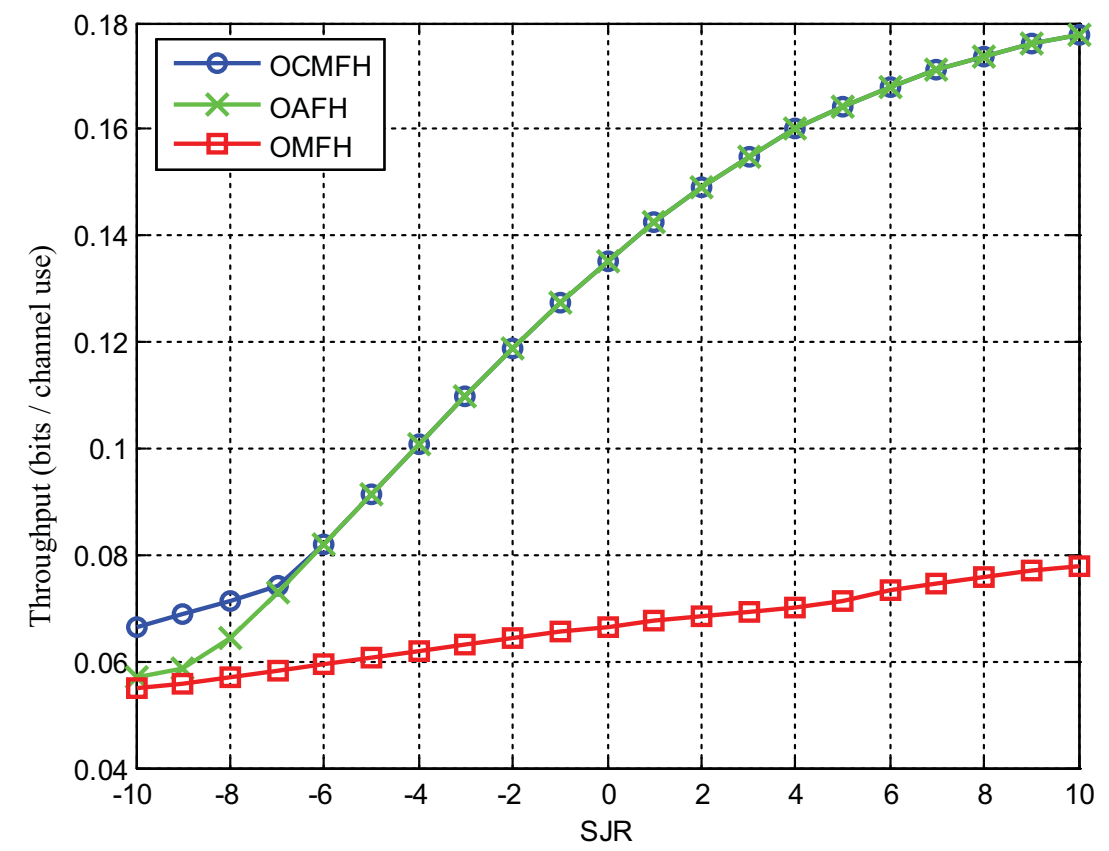

Figure 3.32: Throughput comparison of OMFH, OCMFH and OAFH when the SNR $=-5 \mathrm{~dB}$.

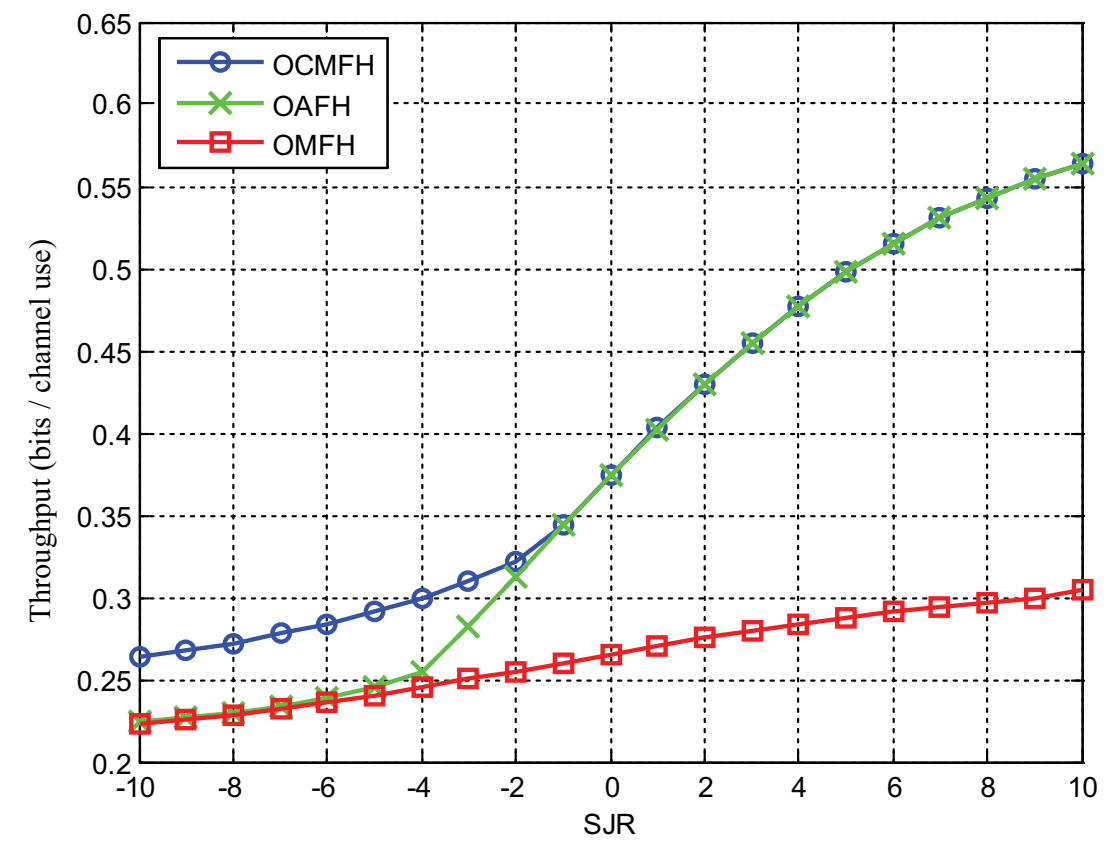

Figure 3.33: Throughput comparison of OMFH, OCMFH and OAFH when the SNR $=0 \mathrm{~dB}$. 


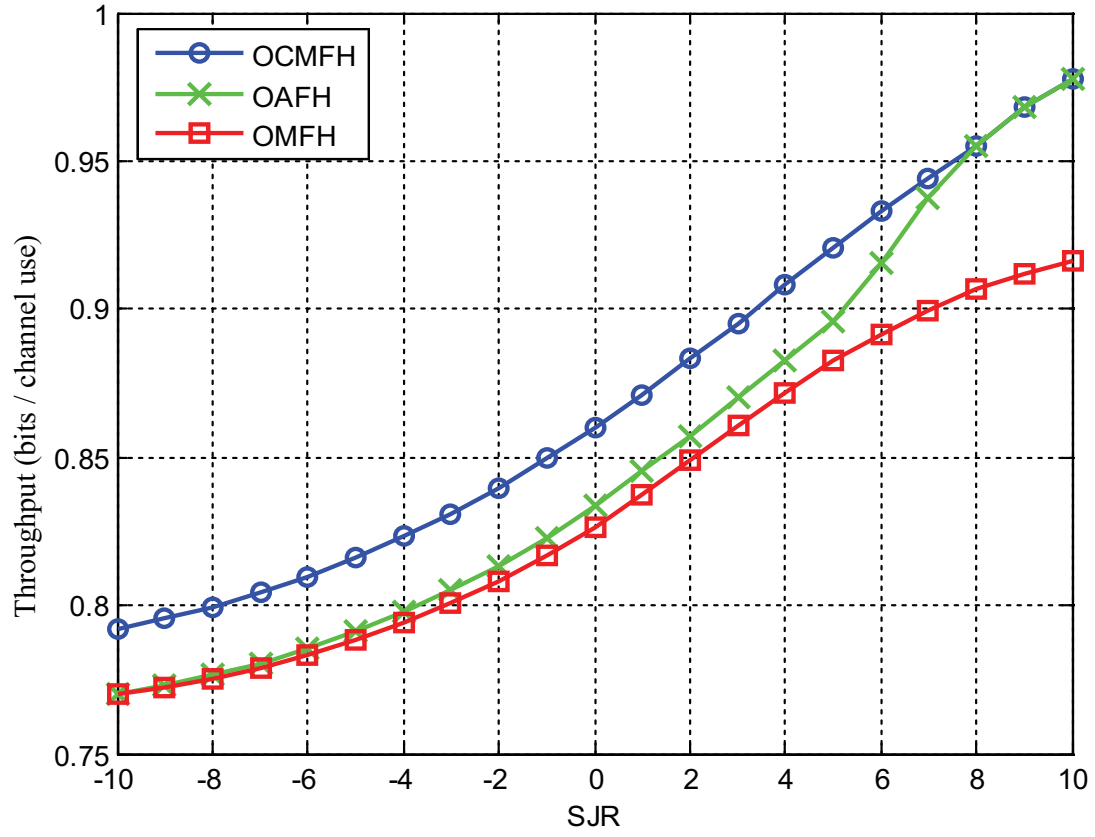

Figure 3.34: Throughput comparison of OMFH, OCMFH and OAFH when the SNR $=10 \mathrm{~dB}$.

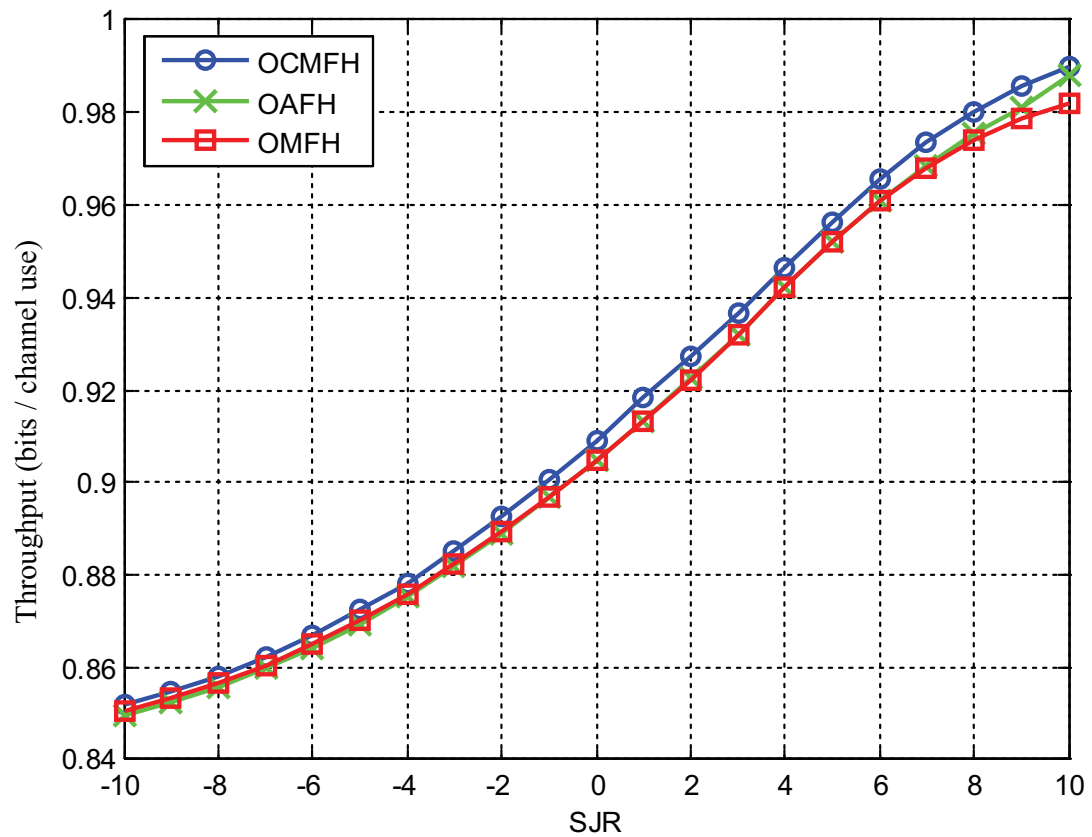

Figure 3.35: Throughput comparison of OMFH, OCMFH and OAFH when the SNR $=15 \mathrm{~dB}$. 


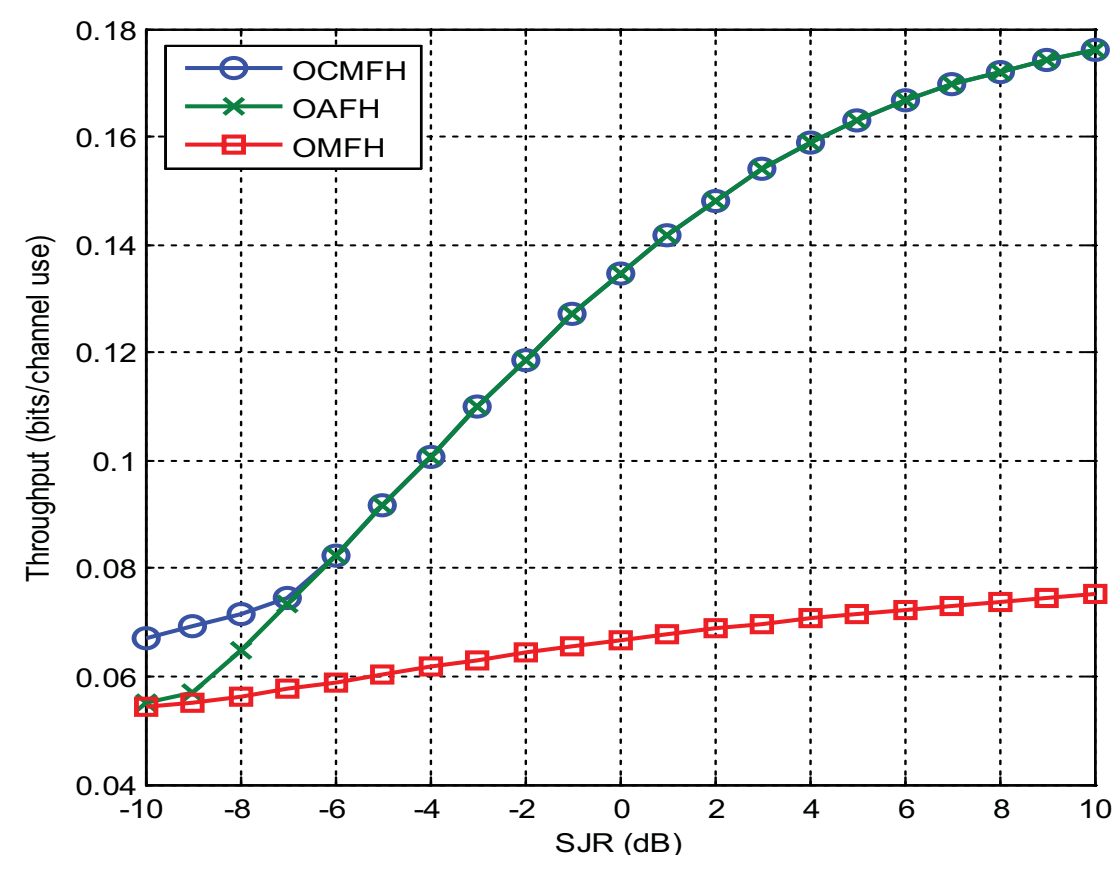

Figure 3.36: Throughput comparison of OMFH, OCMFH and OAFH when the SNR $=-5 \mathrm{~dB}$ in the presence of D1-LOS WINNER channel model.

\subsection{Performance Comparison in Different Channel Models}

We compared the performance of the optimized frequency hopping schemes using D1-NLOS WINNER channel model, which is used for rural environment and with no-line-of-sight (NLOS) between the transmitter and the receiver, in the presence of adaptive BPJ. In this section we want to further compare the performance of the proposed frequency hopping schemes in different channel models. The D1-LOS WINNER channel model that is used for rural environments [78] with line-of-sight (LOS) scenario has a wider coherence bandwidth. This channel model is used as a different channel model example to investigate the performance of the proposed schemes. Figs. 3.36 to 3.40 show the throughput performance of the proposed schemes in different SNR values. It is obvious that OCMFH still has the best throughput performance compared to other schemes in the D1-LOS WINNER channel model. 


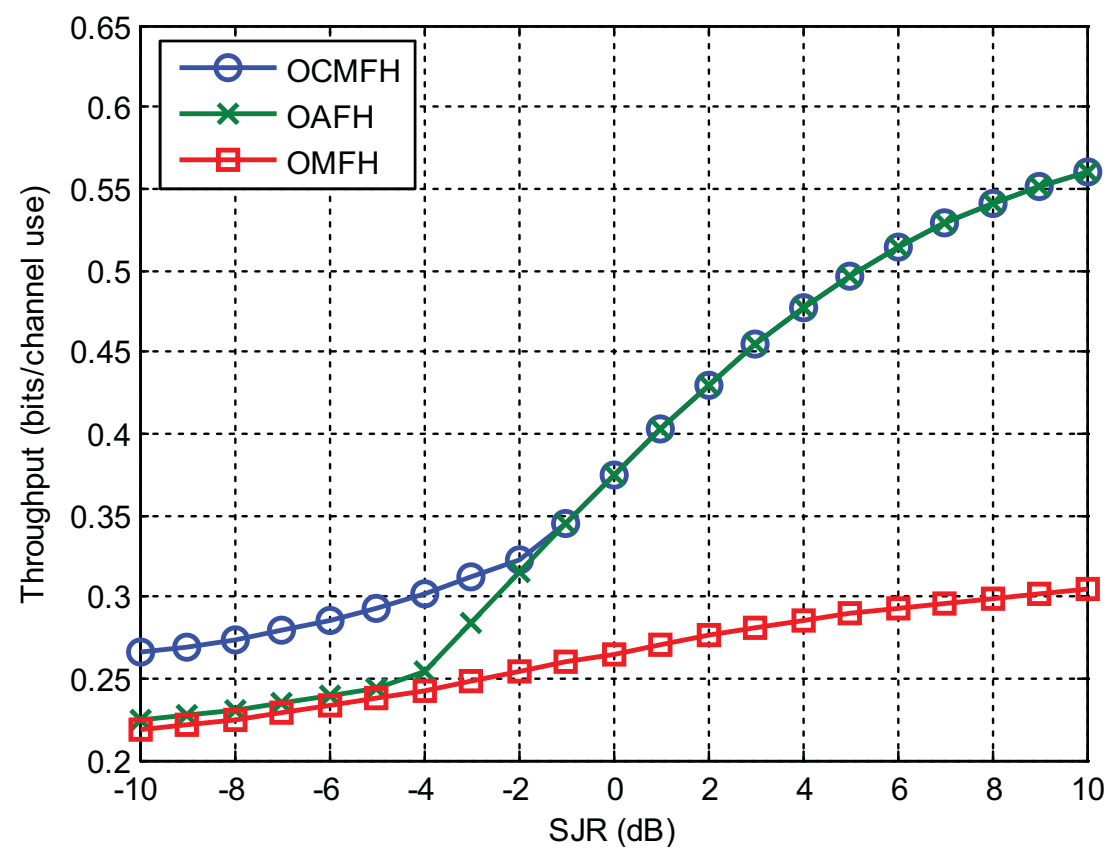

Figure 3.37: Throughput comparison of OMFH, OCMFH and OAFH when the SNR $=0 \mathrm{~dB}$ in the presence of D1-LOS WINNER channel model.

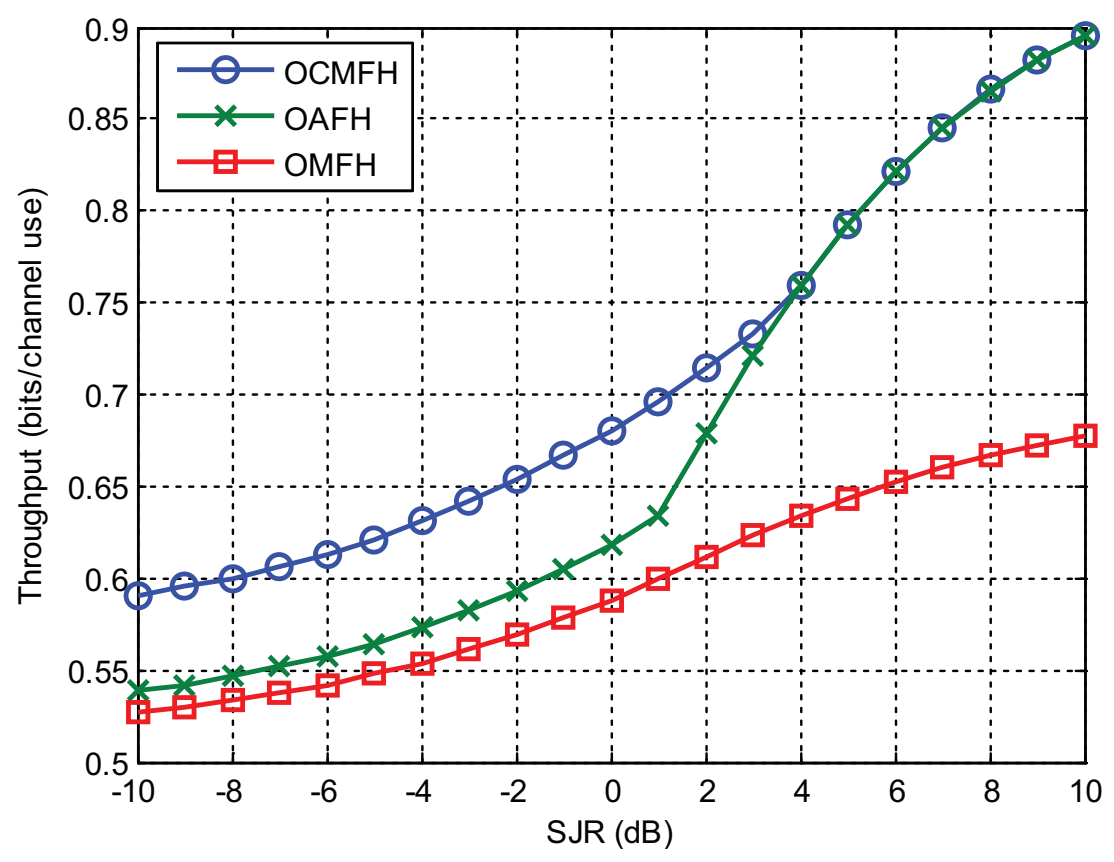

Figure 3.38: Throughput comparison of OMFH, OCMFH and OAFH when the SNR $=5 \mathrm{~dB}$ in the presence of D1-LOS WINNER channel model. 


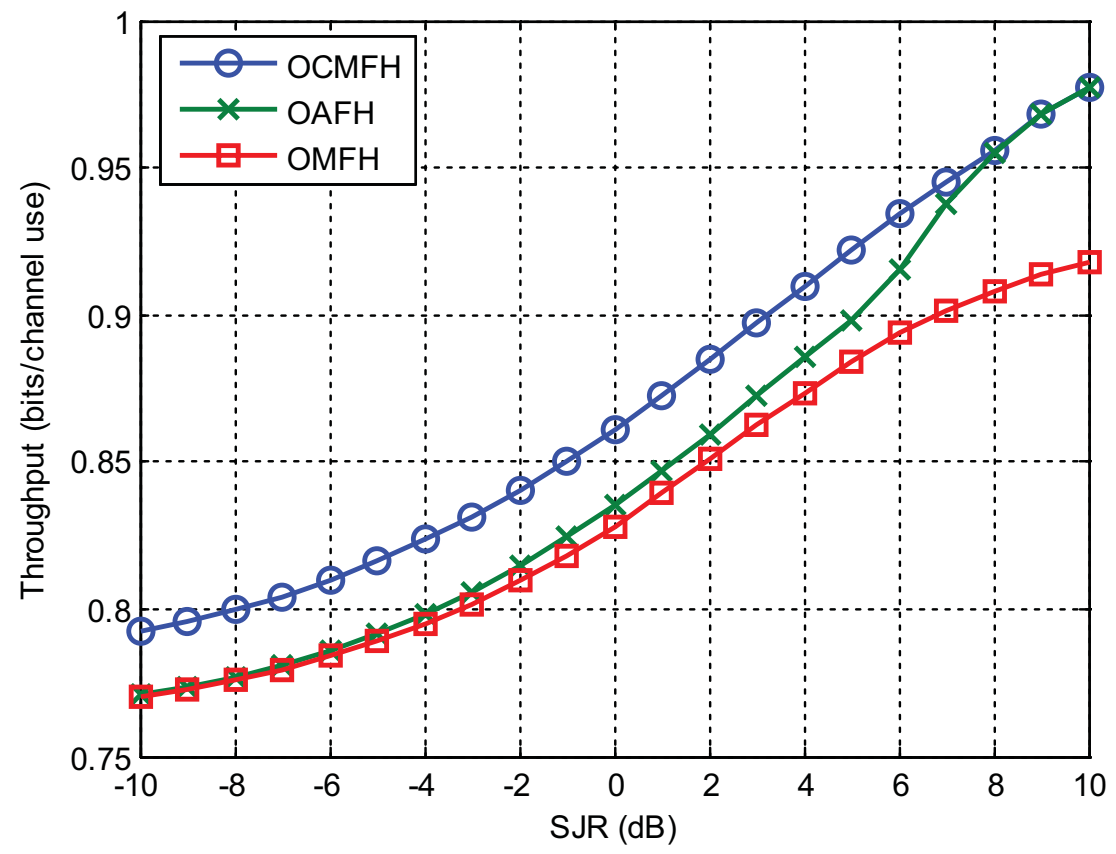

Figure 3.39: Throughput comparison of OMFH, OCMFH and OAFH when the SNR $=10 \mathrm{~dB}$ in the presence of D1-LOS WINNER channel model.

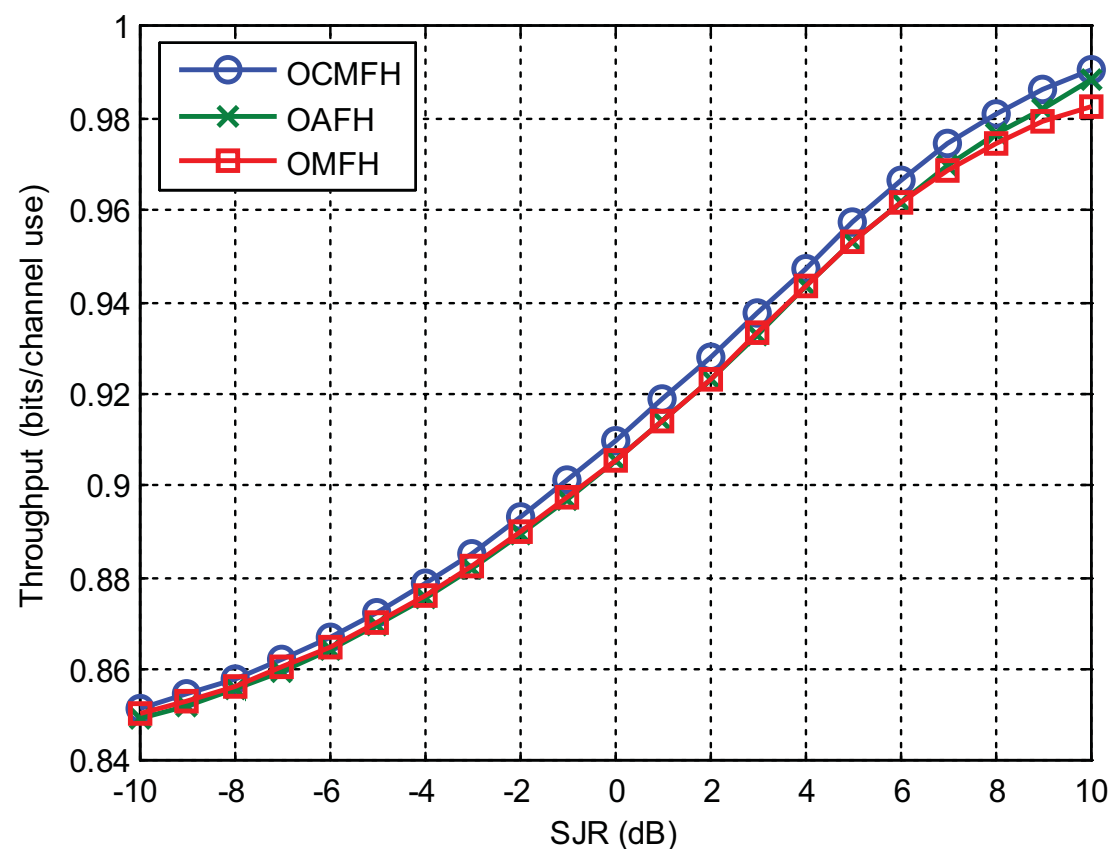

Figure 3.40: Throughput comparison of OMFH, OCMFH and OAFH when the SNR $=15 \mathrm{~dB}$ in the presence of D1-LOS WINNER channel model. 


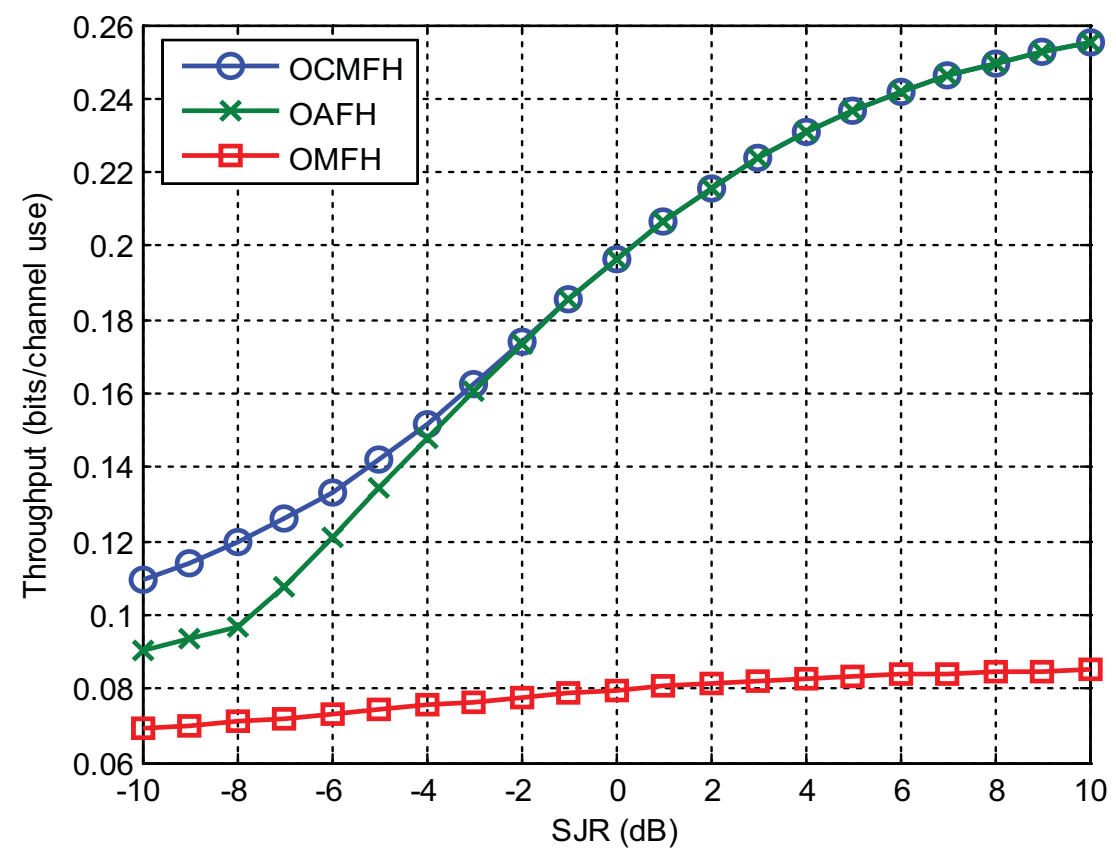

Figure 3.41: Throughput comparison of OMFH, OCMFH and OAFH when the SNR $=-5 \mathrm{~dB}$ in the presence of C2-NLOS WINNER channel model.

An another example of a channel model that has smaller channel coherence bandwidth is the C2-NLOS WINNER channel model. Figs. 3.41 to 3.45 show the throughput performance of the proposed schemes for different SNR values. We can see that the throughput performance of the proposed is the same with different advantages compared to each other.

\subsection{The Effect of Different Jamming Bandwidth}

In this thesis we assumed the presence of adaptive PBJ that jams $10 \%$ of the system transmission bandwidth. We also investigated the throughput performance in the presence of different SNR values and different jamming power scenarios with a signal-to-jamming (SJR) ratio in the range of -10 to $10 \mathrm{~dB}$. In this section we want to investigate the throughput performance of the OCMFH scheme in the presence 


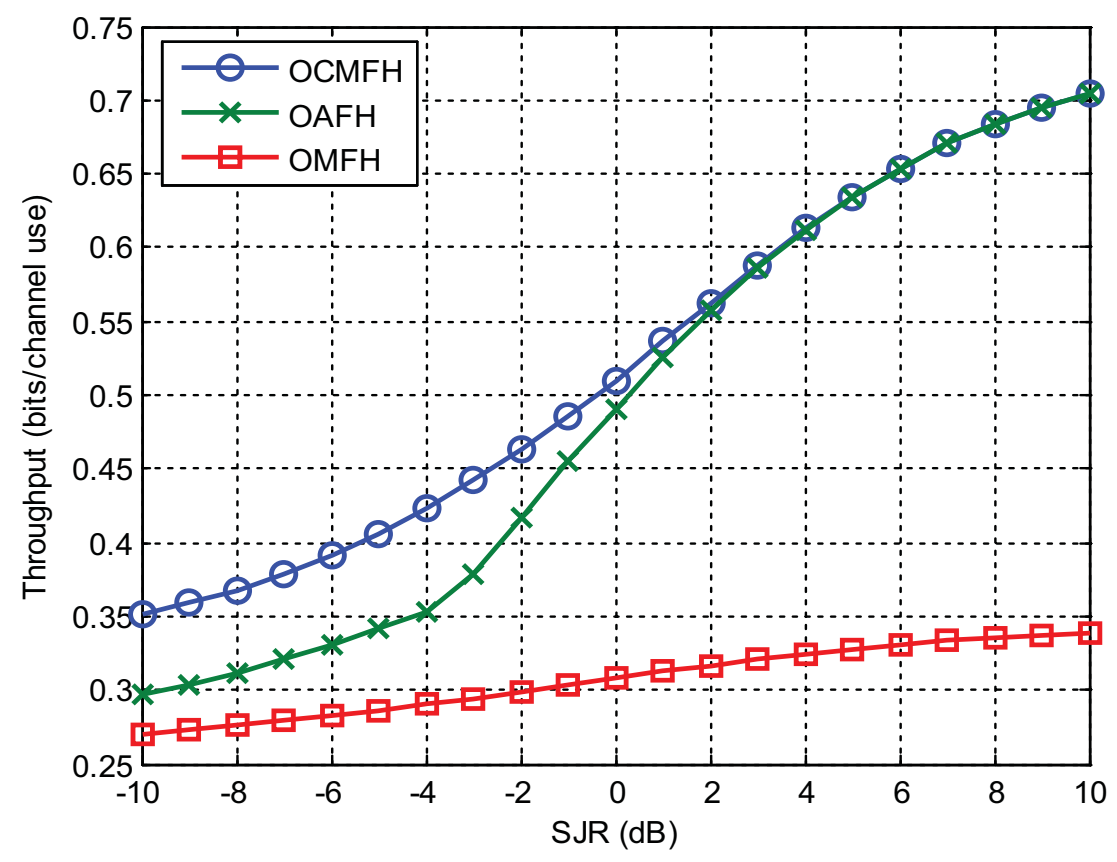

Figure 3.42: Throughput comparison of OMFH, OCMFH and OAFH when the SNR $=0 \mathrm{~dB}$ in the presence of C2-NLOS WINNER channel model.

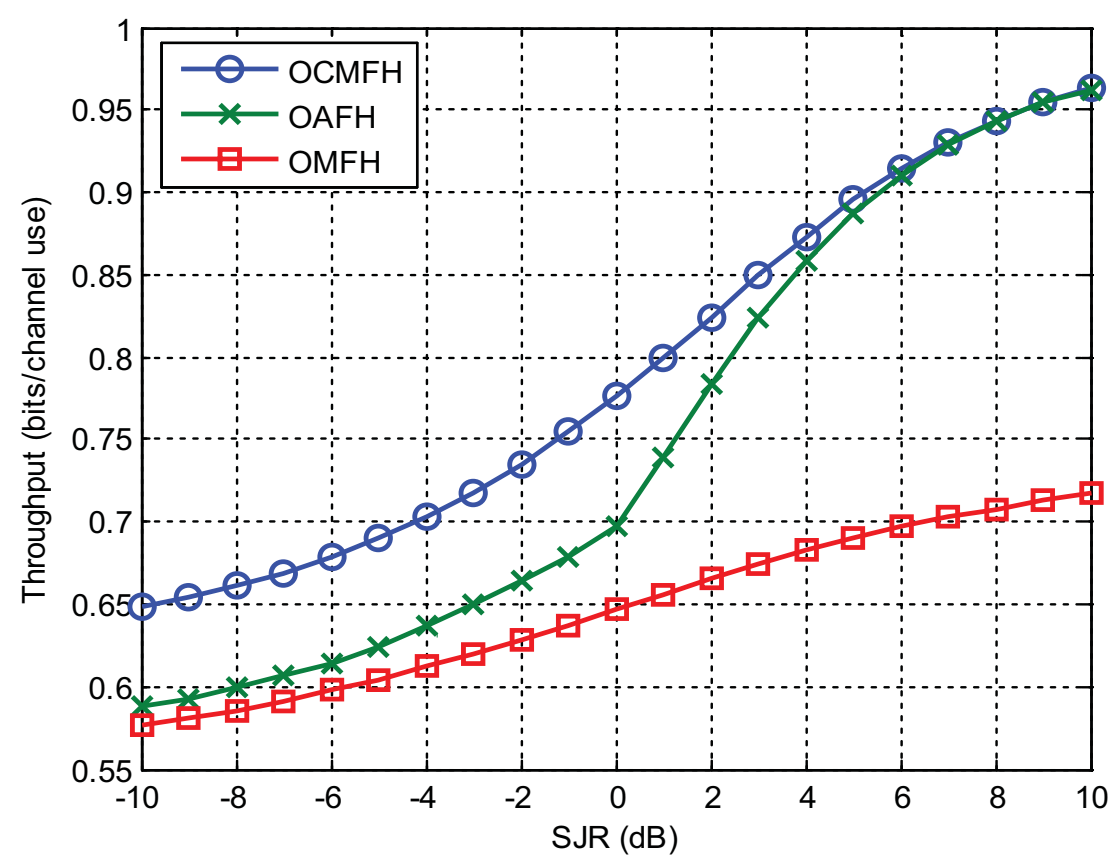

Figure 3.43: Throughput comparison of OMFH, OCMFH and OAFH when the SNR $=5 \mathrm{~dB}$ in the presence of C2-NLOS WINNER channel model. 


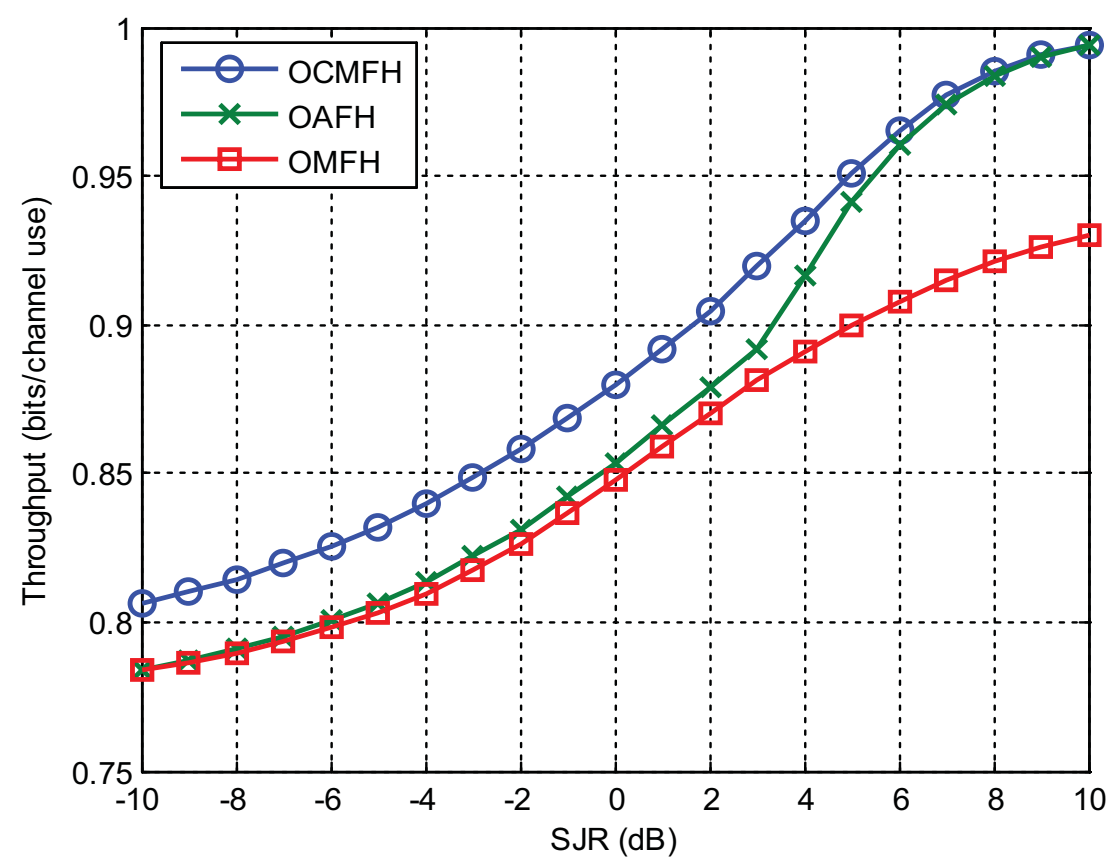

Figure 3.44: Throughput comparison of OMFH, OCMFH and OAFH when the SNR $=10 \mathrm{~dB}$ in the presence of C2-NLOS WINNER channel model.

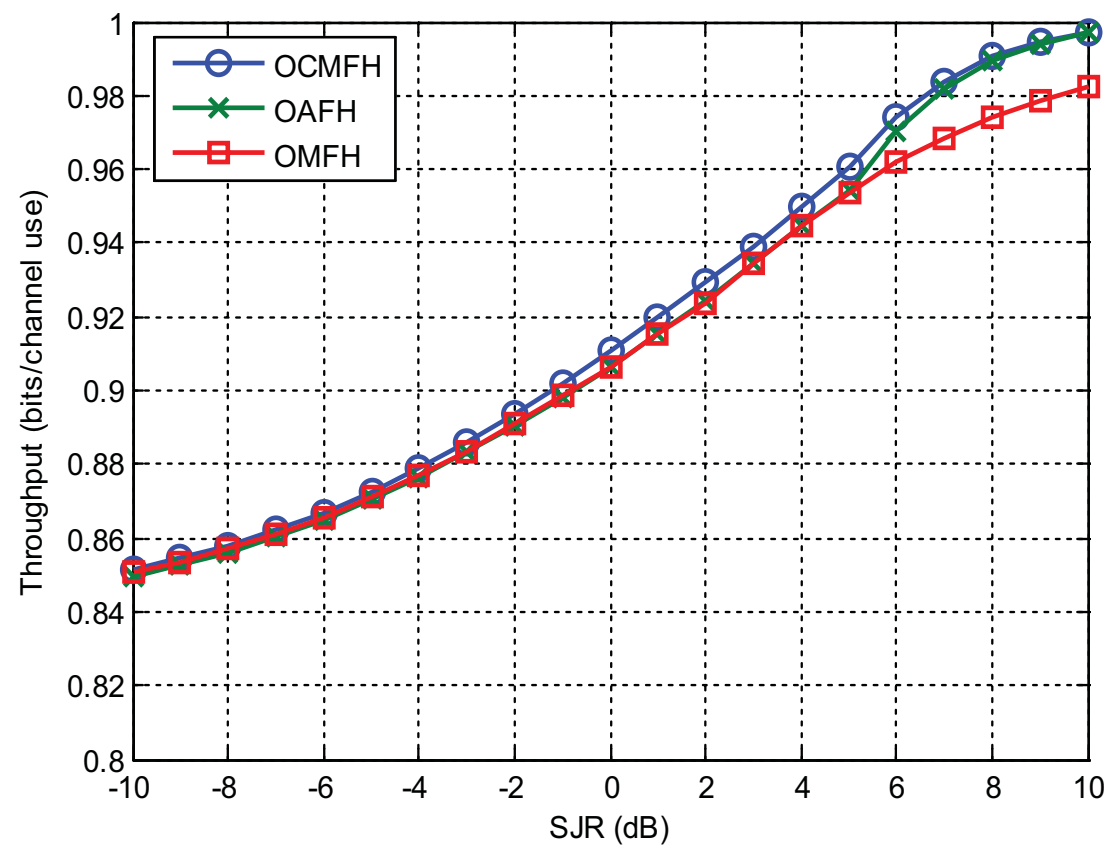

Figure 3.45: Throughput comparison of OMFH, OCMFH and OAFH when the SNR $=15 \mathrm{~dB}$ in the presence of C2-NLOS WINNER channel model. 


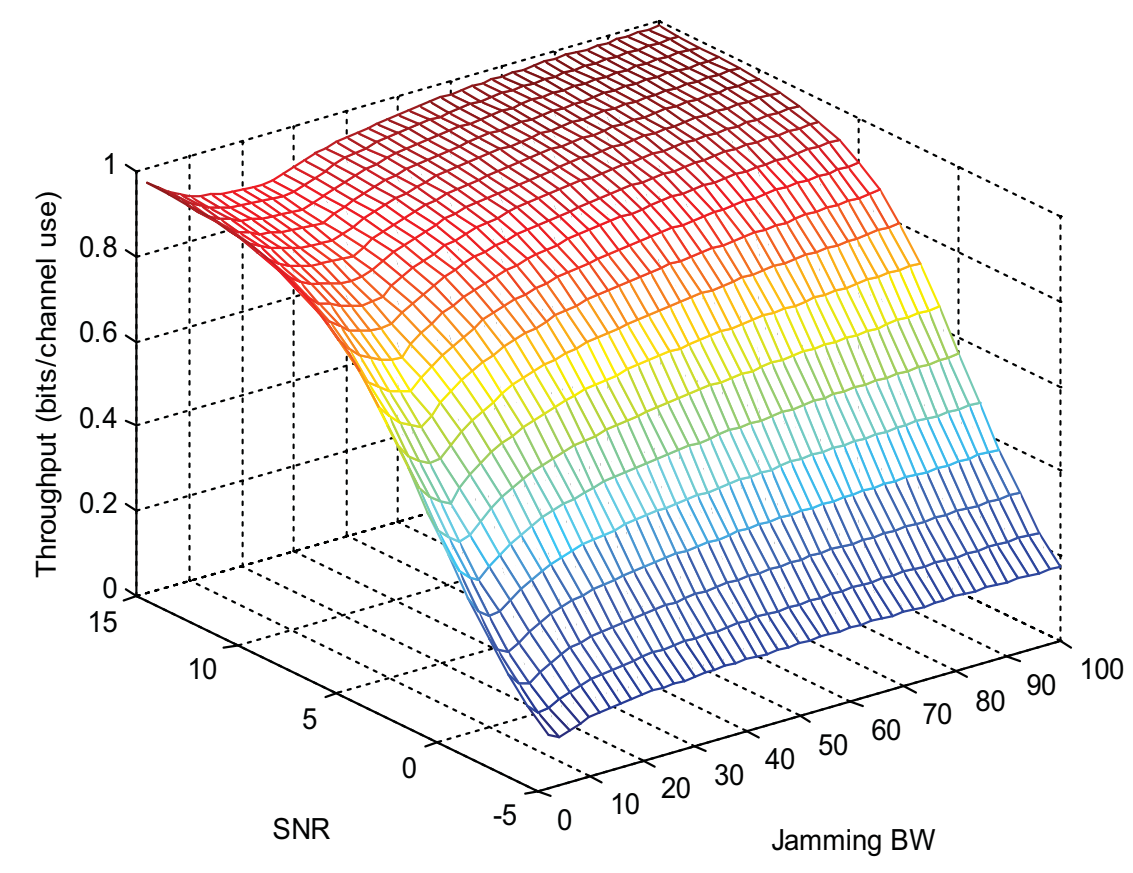

Figure 3.46: The optimum throughput for OCMFH with the presence of different jamming bandwidth and SNR scenarios.

of adaptive PBJ with different jamming bandwidths. Any jammer has a limited jamming power, so when the jammer tries to jam the entire system bandwidth it will be compelled to distribute its jamming power over the total system transmission bandwidth. However, when it tries to jam only one sub-band it can concentrate all its jamming power to jam this sub-band. We assumed that the jammer concentrates its jamming power to jam one sub-band with $\mathrm{SJR}=-10 \mathrm{~dB}$ and it jams the entire band with $\mathrm{SJR}=10 \mathrm{~dB}$ to figure out what is the best jamming bandwidth choice for the jammer to disrupt the communication system transmission. Fig. 3.46 shows the throughput performance of OCMFH in the presence of different SNR values and different jamming bandwidth scenarios.

We can notice that the best jamming bandwidth choice is $10 \%$ of the transmission bandwidth when the SNR has low value as $-5 \mathrm{~dB}$ and it is better to choose $20 \%$ of transmission bandwidth when the SNR is high as $15 \mathrm{~dB}$. 


\subsection{Conclusions}

Changing the control parameters of the original frequency hopping schemes, $\mathrm{MFH}, \mathrm{CMFH}$ and $\mathrm{AFH}$, has a great impact on their performance. By optimizing the hopping set size, $M$, the threshold value, $T$, and the regulation value, $\alpha$, we have obtained a significant throughput improvement over the original schemes. By comparing the optimized schemes we can notice that the OCMFH has the best throughout performance in different SNR and SJR values compared to OAFH and OMFH schemes in the presence of adaptive PBJ in frequency selective fading. 


\section{Chapter 4}

\section{Weighted Random Frequency Hopping}

In all the frequency hopping schemes presented in Chapter 2 and optimized in Chapter 3, a reduced-size hopping set is used. By only using sub-bands with high gain, better throughput is possible. However, use of a reduced-size hopping set means it is possible for an adaptive jammer to jam a larger fraction of the hopping set, which reduces the throughput.

To provide better protection against adaptive jamming, while also exploiting knowledge of the channel gains, we propose a new channel-aware frequency hopping scheme, named weighted random frequency hopping (WRFH), which is described in this chapter.

\subsection{Weighted Random Frequency Hopping}

The WRFH scheme uses the same random code generator that is used in RFH, but the frequency table is different. Instead of using a fixed frequency table, the frequency table is dynamic and is changed according to the channel characteristics. In the WRFH scheme the hopping set contains all of the $K$ sub-bands, just like with RFH. But unlike all of the other schemes, when randomly hopping over the subbands in the hopping set, it does not use a uniform distribution for selecting the next

sub-band. Instead, a non-uniform probability is used so that strong sub-bands are 
more likely to be selected than severely attenuated ones. The probability of selecting sub-band $k$ is chosen to be

$$
P_{k}=\frac{\left|H_{k}\right|^{2}}{\sum_{l=1}^{K}\left|H_{l}\right|^{2}} .
$$

One method for selecting the sub-band to use for each hop based on the probability distribution of (4.1) is to first calculate the cumulative distribution function, given by

$$
C_{k}=\sum_{l=1}^{k} P_{l} .
$$

The index of the sub-band used during the $n^{\text {th }}$ hop is given by the value of $k$ such that $C_{k-1} \leq y_{n}<C_{k}$, where $y_{n}$ is a pseudo-random number selected uniformly over $[0,1)$

Because the hopping set used by WRFH contains all $K$ sub-bands, the adaptive jammer described in Chapter 3 is not able to effectively disrupt communication. The jammer would essentially end up jamming two arbitrarily-selected disjoint clusters of sub-bands, and therefore would be non-adaptive. To provide a more fair comparison it is necessary to assume that the jammer can be re-programmed to use a different cluster-selection algorithm, one that is more effective against WRFH.

If the jammer listens to the channel before it commences jamming it could estimate the hopping probability for each sub-band, and then try to jam as many of the most-likely-to-be-used sub-bands as possible. However, to accurately estimate these probabilities, the jammer would have to listen for a long time, and during this time it would not be able to jam anything. On the other hand, if the jammer does not listen for long enough, inaccurate estimation of the probabilities may lead the jammer to 
jam seldomly-used sub-bands. To provide a compromise, we assume that the jammer is able to listen for long enough to determine which sub-bands are more likely to be used, and tries to jam as many of these as possible. More precisely, we assume that the jammer tries to jam as many sub-bands that have a greater than average hopping probability (i.e., sub-bands with $P_{k}>\frac{1}{K}$ ).

We will investigate the throughput performance of WRFH in the absence of jamming and in presence of infinite and finite PBJ over a frequency selective fading channel. The average throughput that has been used to compare the different frequency hopping schemes, based on the throughput of noncoherently detected binary FSK over an additive white Gaussian noise channel with a signal-to-noise ratio (SNR) of $\gamma$, was presented in Chapter 3 and is given by (2.10). With WRFH, all sub-bands can possibly be selected, and the probability of selecting sub-band $k$ is $P_{k}$, given by (4.1), so the average throughput for a given channel realizations $\underline{\underline{H}}_{S}$ and $\underline{\underline{H}}_{J}$ is

$$
\eta\left(\underline{\underline{H}}_{S}, \underline{\underline{H}}_{J}, \gamma\right)=\sum_{k=1}^{K} \eta\left(\rho_{k}\right) P_{k} .
$$

where $\rho_{k}$ is the instantaneous SJNR on sub-band $k$. In this case the average throughput, averaged over all channel realizations, is given by (2.13). We can evaluate (2.13) numerically using Monte Carlo integration, the same way that we did in Chapter 3. The throughput performance of WRFH in the presence of different finite jamming power scenarios and SNR values is shown in Fig. 4.1.

The throughput performance of WRFH is compared to OMFH and RFH to show the advantages of using WRFH. Fig. 4.2 shows that OMFH has the best throughput performance in the absence of jamming. This is because, in the absence of jamming, the OMFH algorithm only uses the sub-band that has the highest channel gain. With WRFH all sub-bands are used but the sub-bands with high channel gains are used 


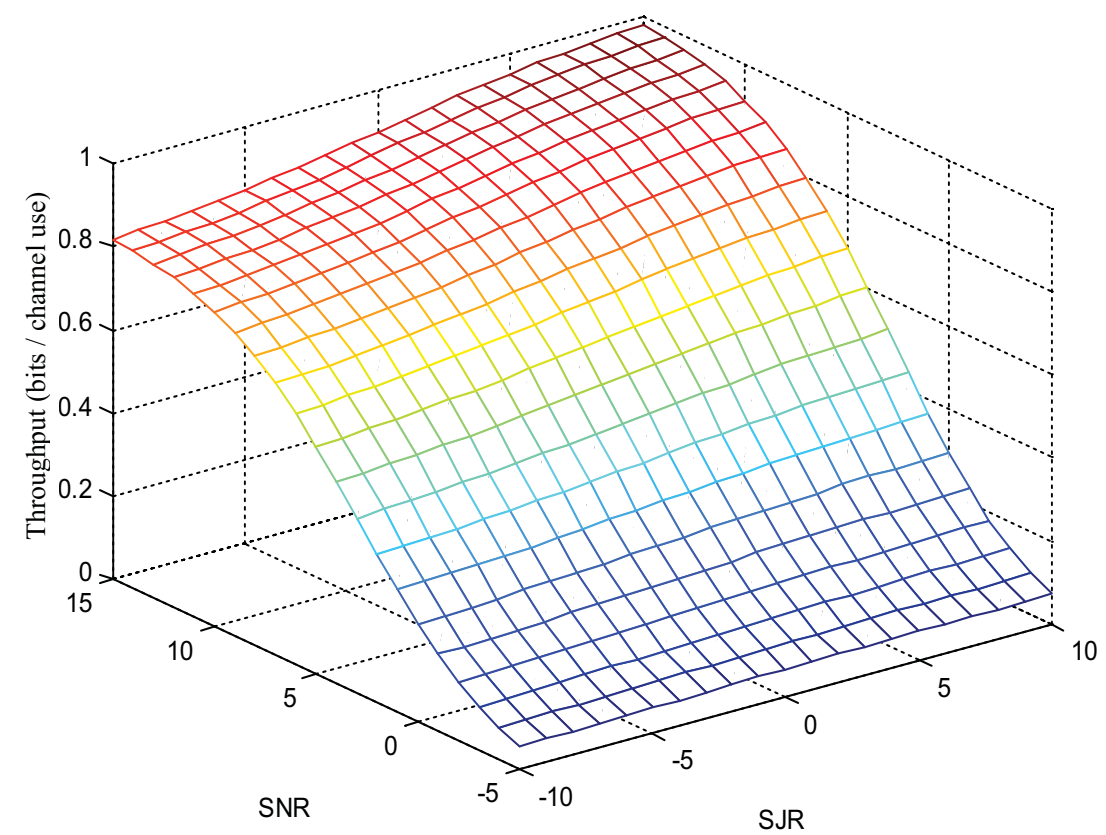

Figure 4.1: The performance of WRFH in different jamming power and SNR scenarios.

most of the time. This makes its performance slightly less than OMFH. However, WRFH has much better throughput performance than RFH, because RFH does not consider the channel characteristics and it selects the sub-bands uniformly from all the available sub-bands $K$.

By investigating the performance of WRFH in the presence of weak jamming as shown in Fig. 4.3, we notice that the performance gap between WRFH and OMFH decreases and they have almost the same performance. WRFH still has better performance than RFH. Increasing the jamming power further, as in Figs. 4.4 and 4.5, illustrates the advantage of using WRFH.

In the presence of strong jamming WRFH has better throughput performance than OMFH and RFH because, as the jamming power increases, the OMFH algorithm tries to select a large number of sub-bands to reduce the jamming effect. This gives a higher throughput than using only a few sub-bands, but forces the algorithm to select 


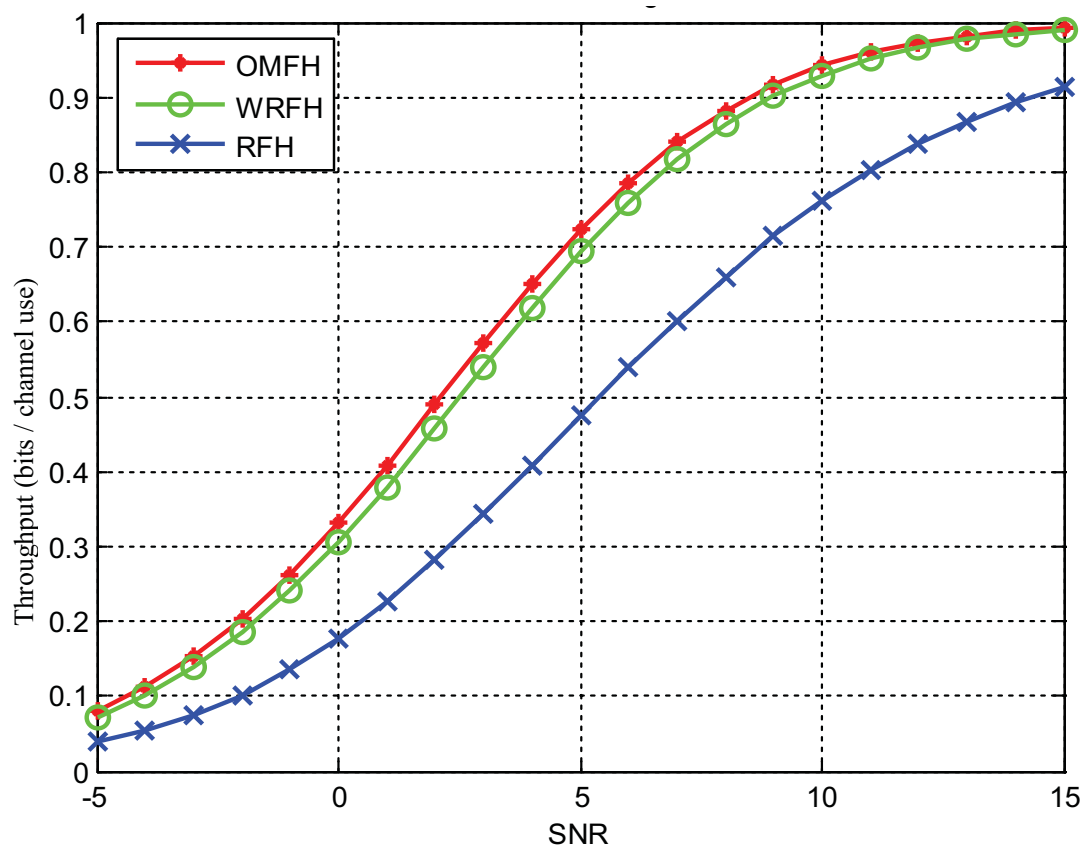

Figure 4.2: The performance of WRFH compared to OMFH and RFH in the absence of jamming.

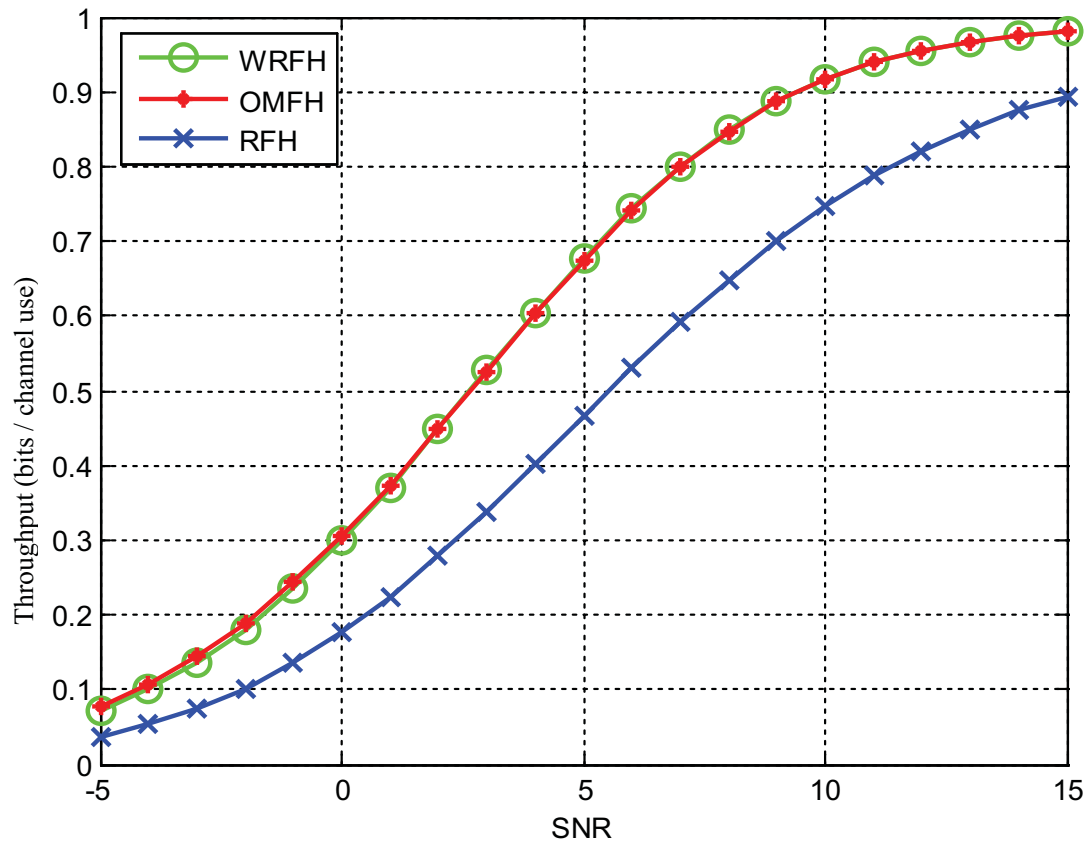

Figure 4.3: The performance of WRFH compared to $\mathrm{OMFH}$ and $\mathrm{RFH}$ when $\mathrm{SJR}=10$ dB. 


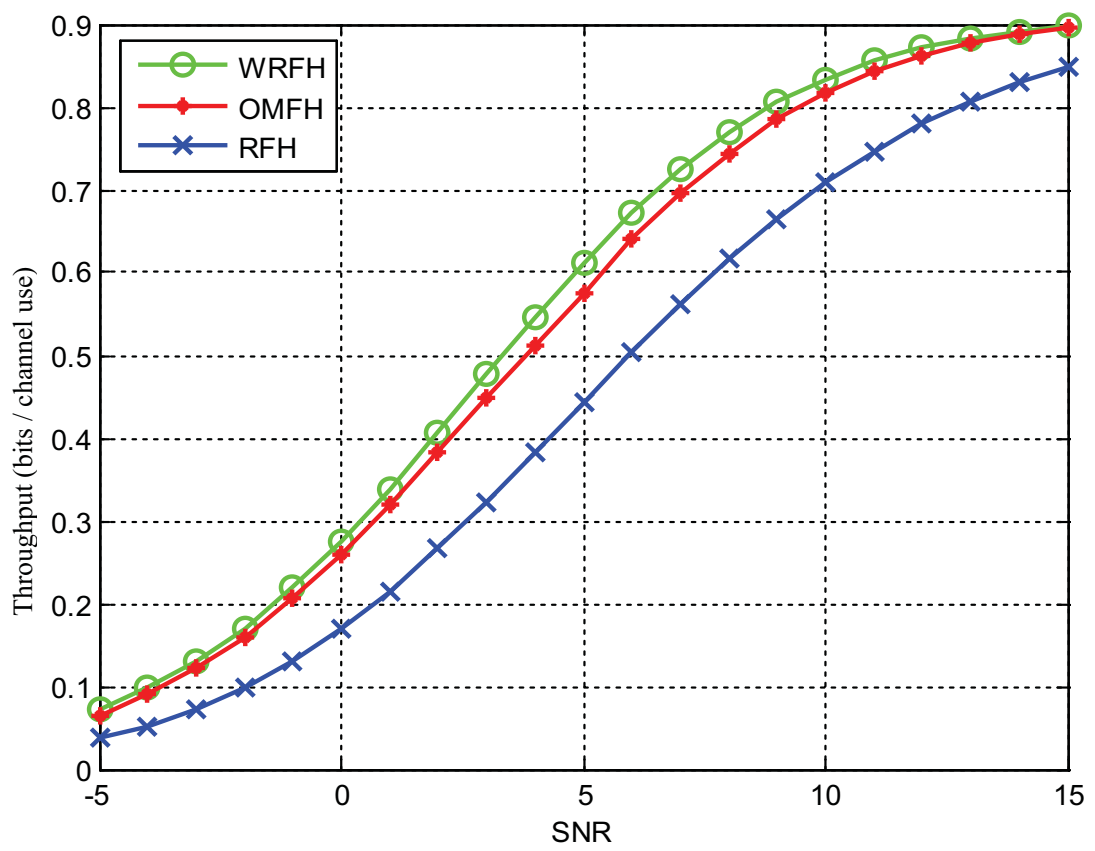

Figure 4.4: The performance of WRFH compared to OMFH and RFH when SJR $=0$ dB.

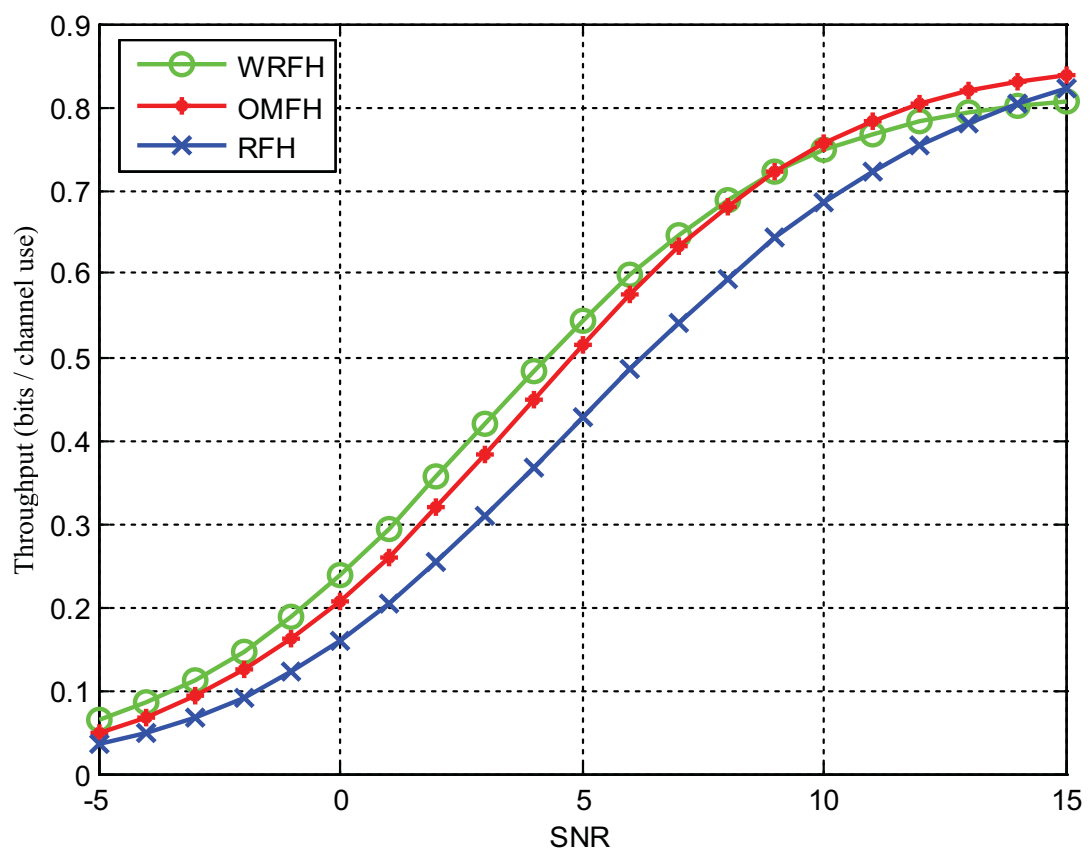

Figure 4.5: The performance of WRFH compared to OMFH and RFH with the presence of infinite jamming. 
the sub-bands with low channel gains. These are used with the same probability as the sub-bands with high channel gains. On the other hand, WRFH uses all the sub-bands but sub-bands with higher channel gains are used more often while subbands with lower channel gains are seldom used, making WRFH more efficient in the presence of jamming. RFH has the worst throughput performance either in the absence or in the presence of jamming because it selects the sub-bands uniformly from the whole available sub-bands without considering the channel characteristics, degrading the throughput performance.

Although WRFH is generally better than OMFH when the jamming is strong, this is not true when the SNR is high as well, as can be seen in Fig. 4.5. This cross-over in performance is caused by the different adaptive jamming strategy used against WRFH. When the jamming power is strong and the SNR is high, the OMFH algorithm tries to select as many sub-bands as it can (i.e., the optimal value of $M$ is 100 as shown in Fig. 3.7). The more sub-bands that are in the hopping set the lower the fraction of the hopping set that is jammed, so the effects of jamming are mitigated. Furthermore, since the throughput of binary FSK saturates at 1 bit per channel use, when the SNR is high most sub-bands have high throughput, so there is very little advantage from using only the very best sub-bands. As a result, OMFH works well when the jamming is strong and the SNR is high. On the other hand, although all $K$ sub-bands are included in the hopping set for WRFH, only those channels with the highest gain are used most of the time, even though other subbands, with lower gains, would give nearly the same throughput when the SNR is high. As a result, the jammer adapting to WRFH is able to jam a large fraction of the most frequently selected sub-bands, thereby limiting the efficiency of WRFH.

By using a hopping set that contains all $K$ sub-bands, but hopping over the 
sub-bands according to a non-uniform probability distribution, WRFH was able to give better performance than OMFH. However, since OAFH and OCMFH also give better performance than OMFH, it should be possible to further improve on the performance of WRFH by incorporating the ideas behind OAFH and OCMFH.

\subsection{Advanced Weighted Random Frequency Hopping}

In advanced weighted random frequency hopping (AWRFH), the system uses all $K$ sub-bands, and uses a non-uniform hopping probability distribution, just like WRFH. However, AWRFH uses the modified channel gains used by AFH,

$$
P_{k}=\frac{\left|H_{k}\right|^{2}}{(1+\alpha) \max _{k}\left|H_{k}\right|^{2}-\left|H_{k}\right|^{2}}
$$

as the hopping probabilities (after normalization to ensure $\sum_{k=1}^{K} P_{k}=1$ ).

To realize the best performance possible, we should adjust the regulation parameter, $\alpha$, depending on the SNR and jamming power. First, we calculated the throughput at each value of $\alpha$ for different jamming power scenarios and SNR values to have a clear vision about the best value of $\alpha$ that achieves the maximum throughput. The throughput at each value of $\alpha$ for different jamming power scenarios when the $\mathrm{SNR}=0,5$ and $10 \mathrm{~dB}$ are shown in Figs. 4.6, 4.7 and 4.8 as an illustrative example.

The optimum regulation value and the optimum throughput are determined by selecting the value of $\alpha$ that achieves the maximum throughput for each jamming power scenario at different SNR values as shown in Figs. 4.9 and 4.10.

To show the throughput advantage of AWRFH we will compare its throughput performance with OAFH and WRFH in the the absence of jamming as shown in 


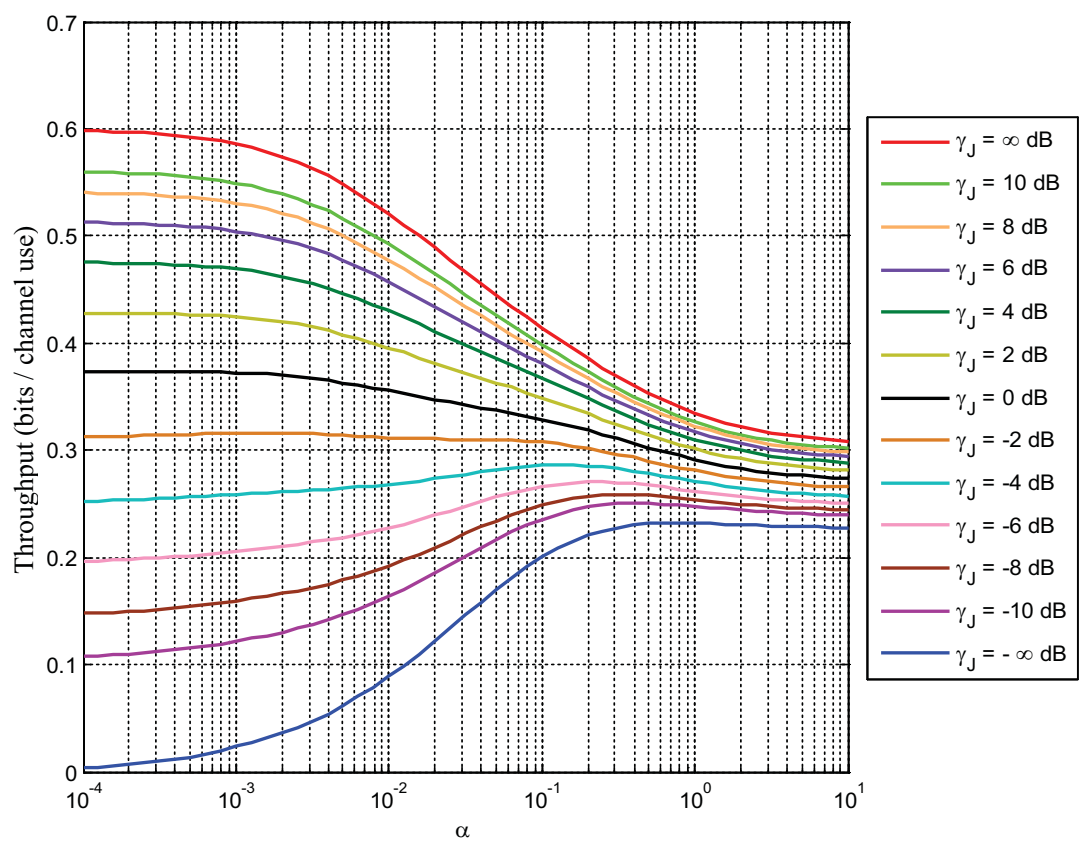

Figure 4.6: The throughput vs. the regulation value for AWRFH with different jamming powers and $0 \mathrm{~dB}$ SNR.

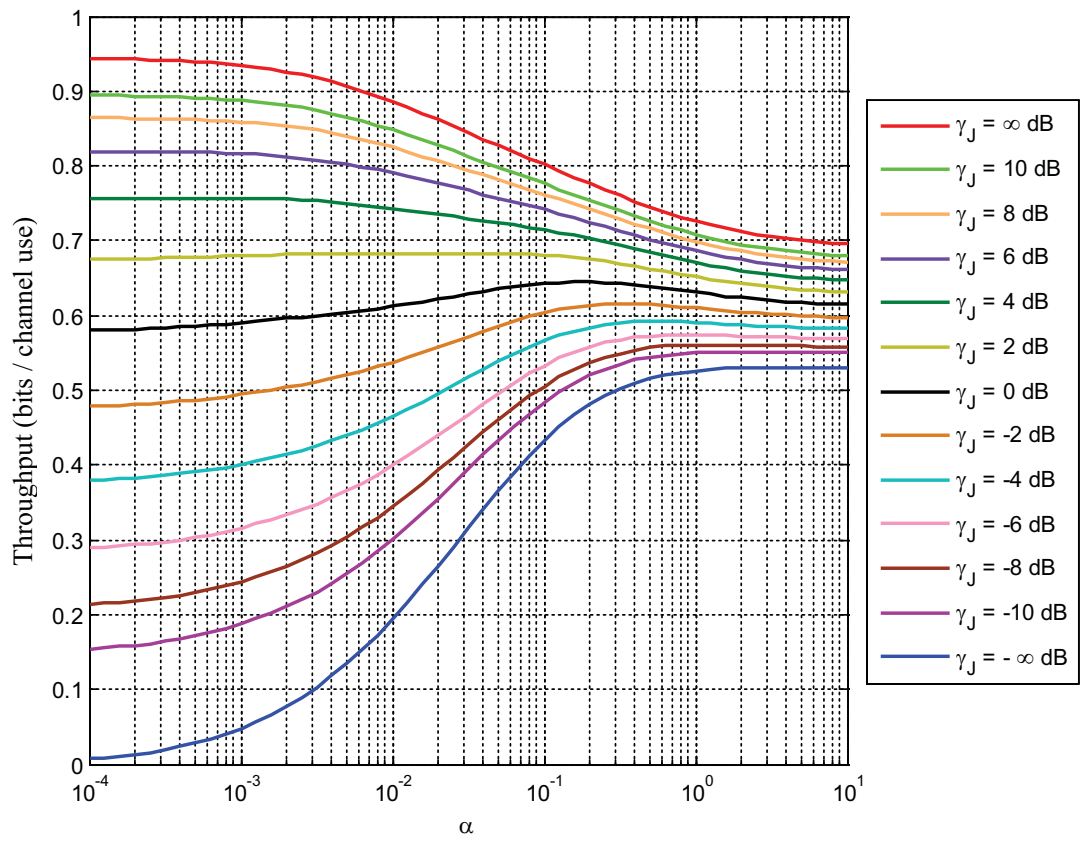

Figure 4.7: The throughput vs. the regulation value for AWRFH with different jamming powers and $5 \mathrm{~dB}$ SNR. 


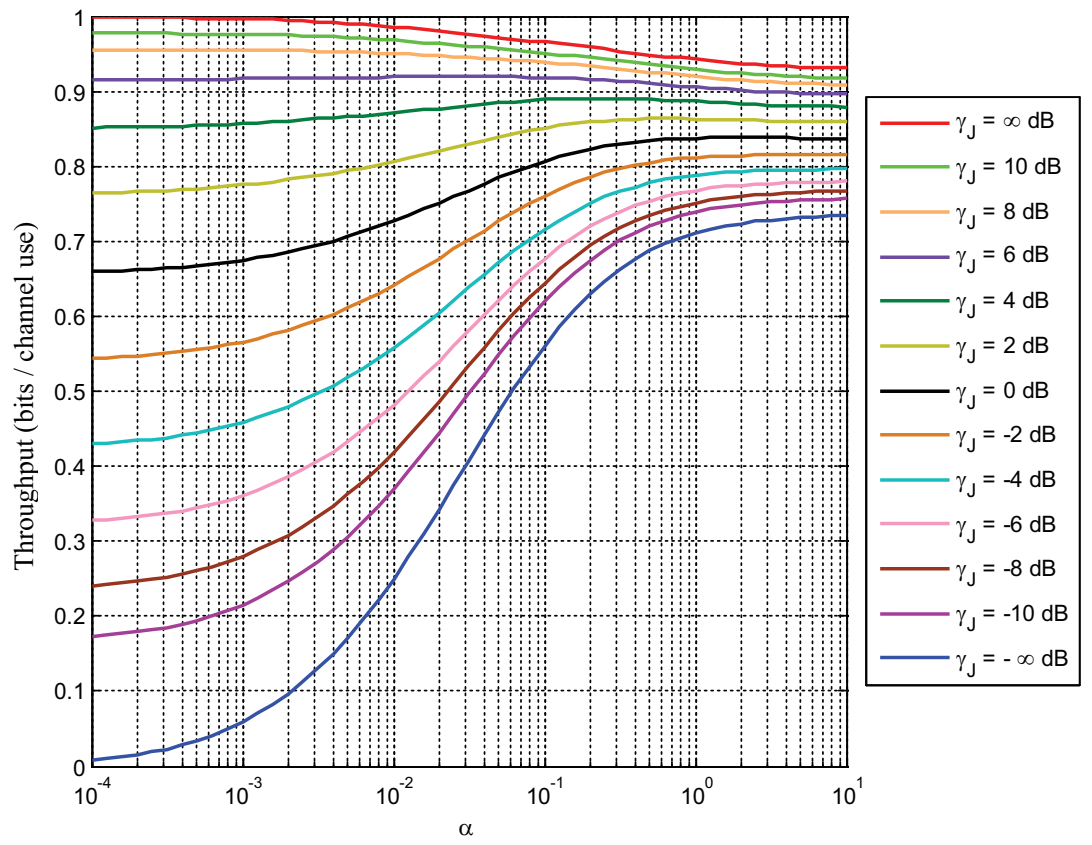

Figure 4.8: The throughput vs. the regulation value for AWRFH with different jamming powers and $10 \mathrm{~dB}$ SNR.

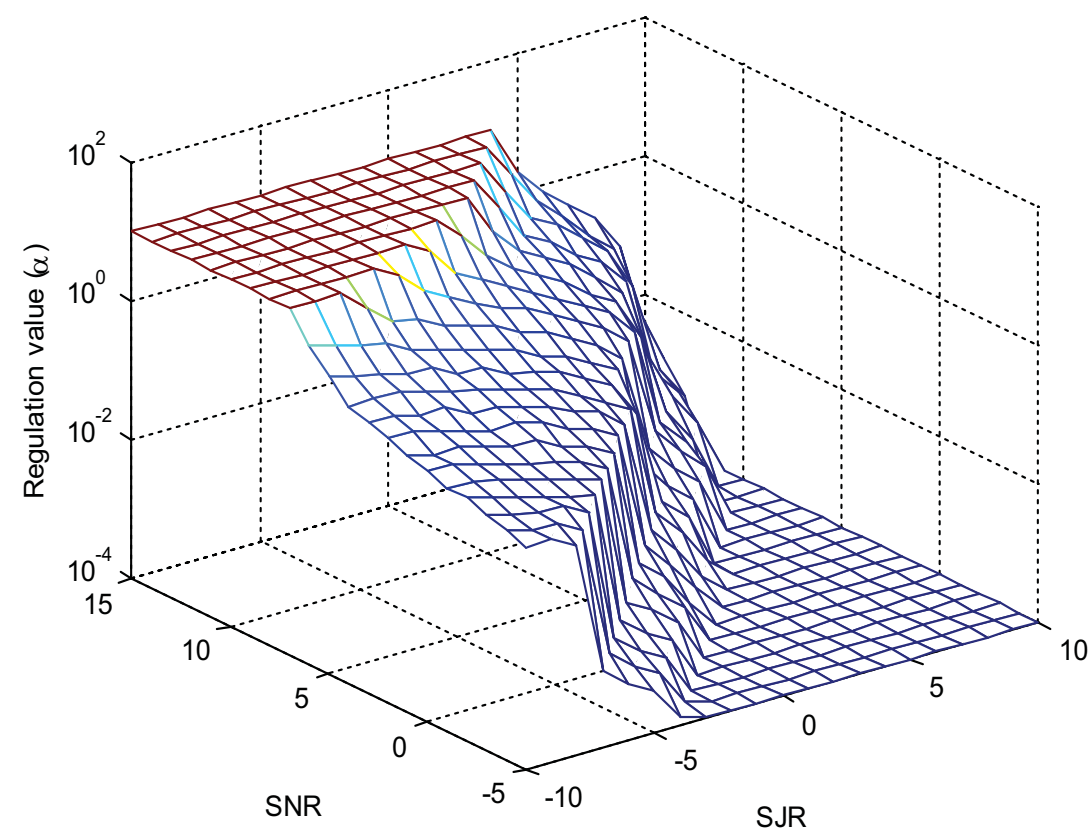

Figure 4.9: The optimum regulation value for AWRFH in different jamming power and SNR scenarios. 


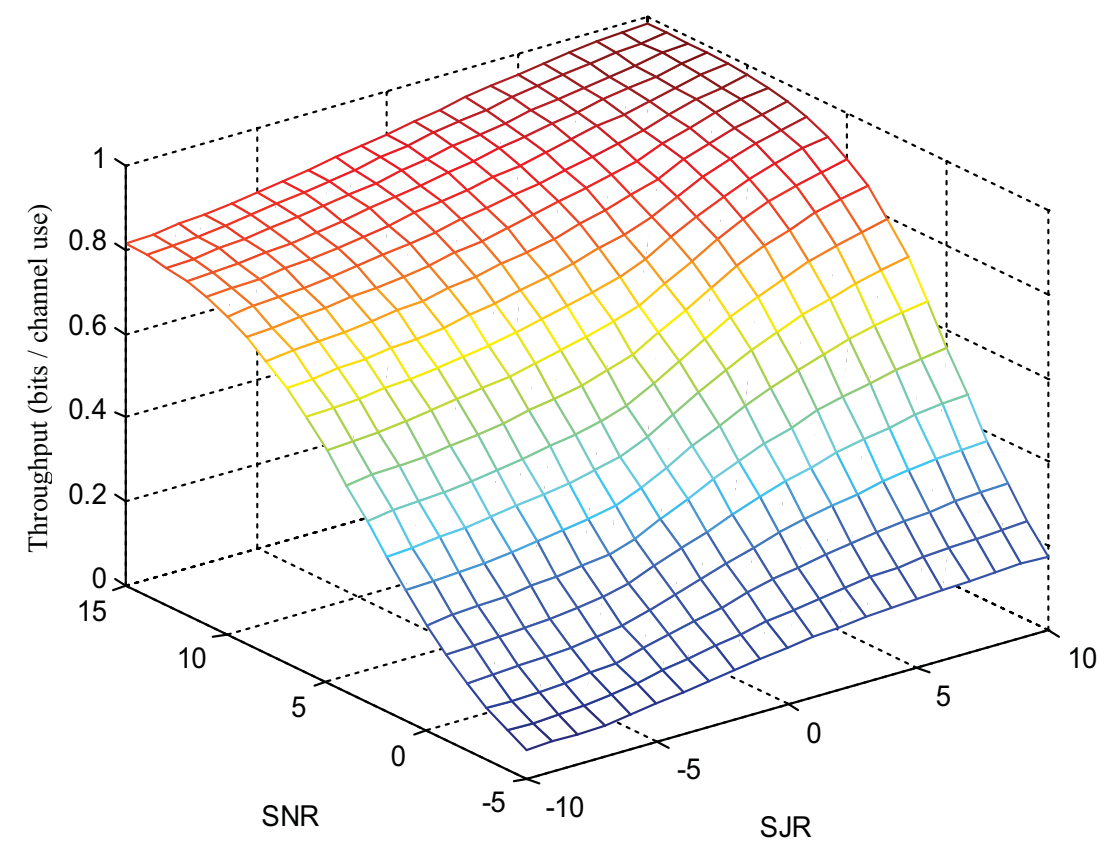

Figure 4.10: The optimum throughput for AWRFH in different jamming power and SNR scenarios.

Fig. 4.11 and in the presence of different examples of jamming power.

We compared the throughput performance of AWRFH with OAFH and WRFH assuming the SJR $=10 \mathrm{~dB}$ an example for weak jamming power, the comparison results will be as shown in Fig. 4.12. We compared their performance in the presence of strong jamming by assuming the $\mathrm{SJR}=0 \mathrm{~dB}$ as shown in Fig. 4.13. Increasing the jamming power to be infinite and comparing the performance of these schemes, the result will be as shown in Fig. 4.14.

We can see that AWRFH has the same performance as OAFH in the absence of jamming because both algorithms select a value of $\alpha$ small enough to force the system to only use the sub-band with highest channel gain. When the jamming starts to appear in the channel the performance of AWRFH becomes slightly better than OAFH. However, both AWRFH and OAFH have much better throughput performance than WRFH in all cases. 


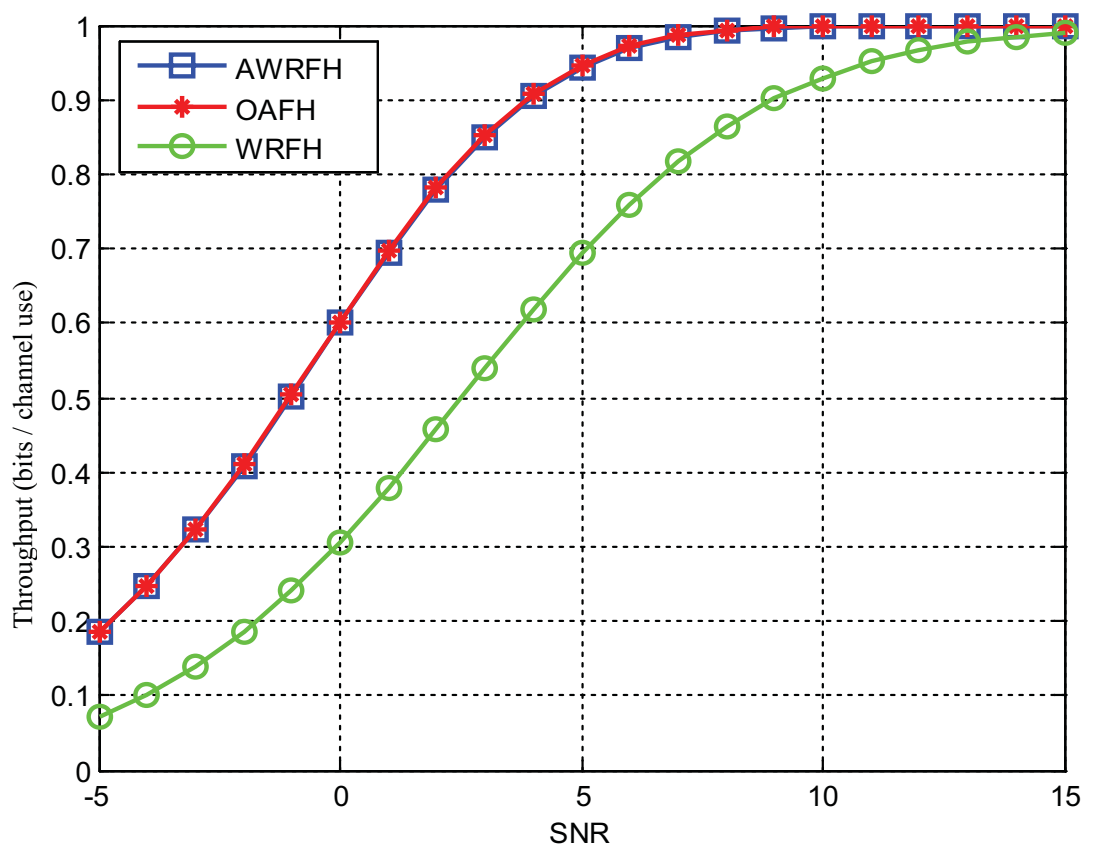

Figure 4.11: The performance of AWRFH compared to OAFH and WRFH in the absence of jamming.

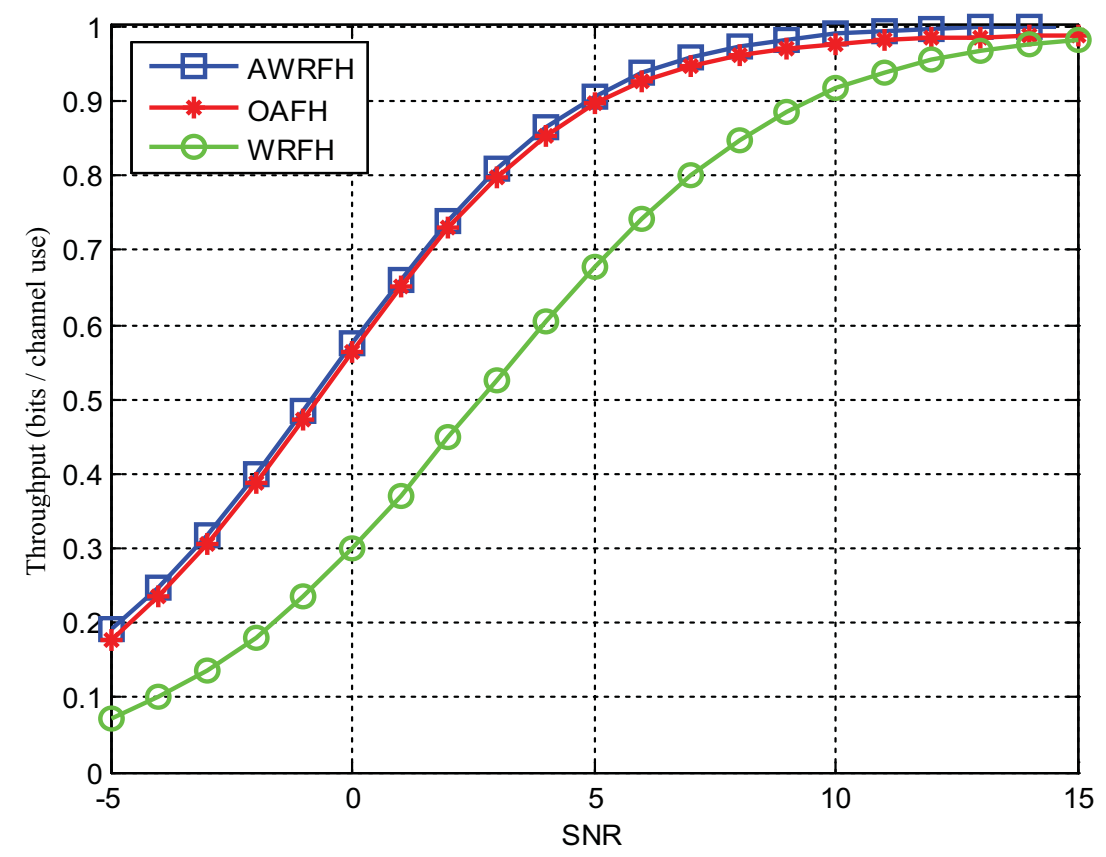

Figure 4.12: The performance of AWRFH compared to OAFH and WRFH when SJR $=10 \mathrm{~dB}$. 


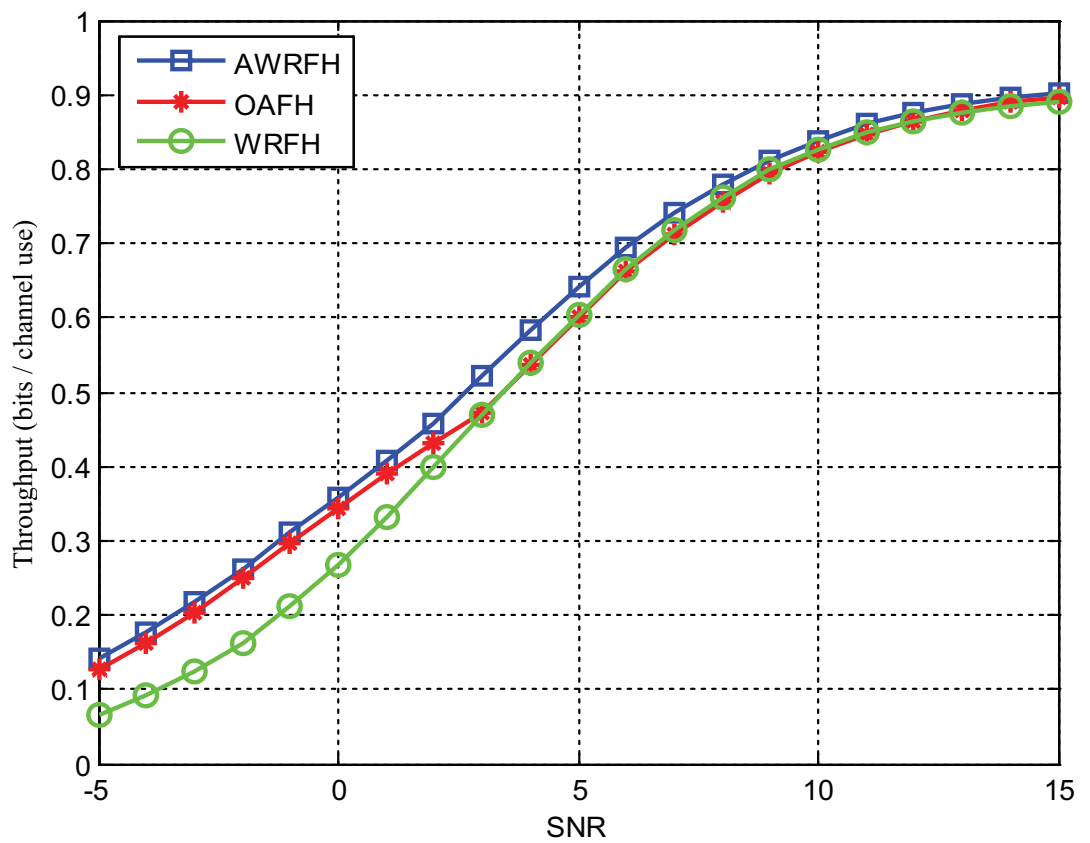

Figure 4.13: The performance of AWRFH compared to OAFH and WRFH when SJR $=0 \mathrm{~dB}$.

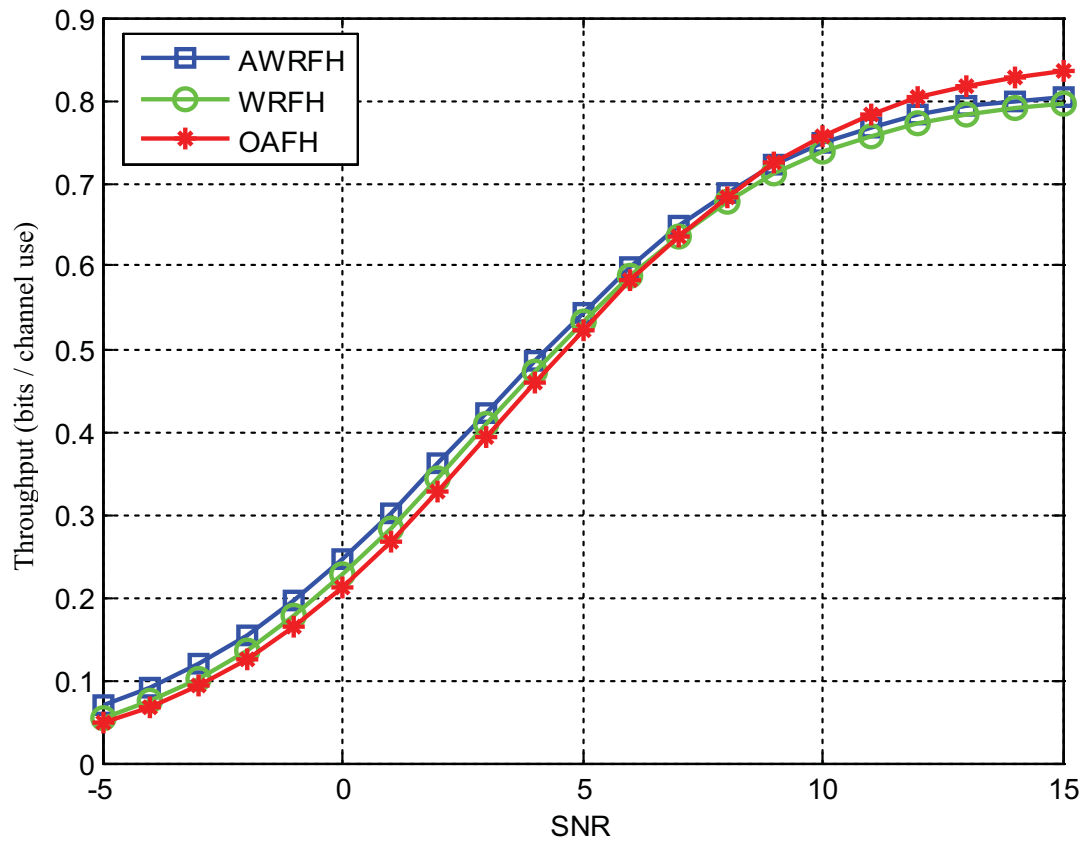

Figure 4.14: The performance of AWRFH compared to OAFH and WRFH in the presence of infinite jamming. 


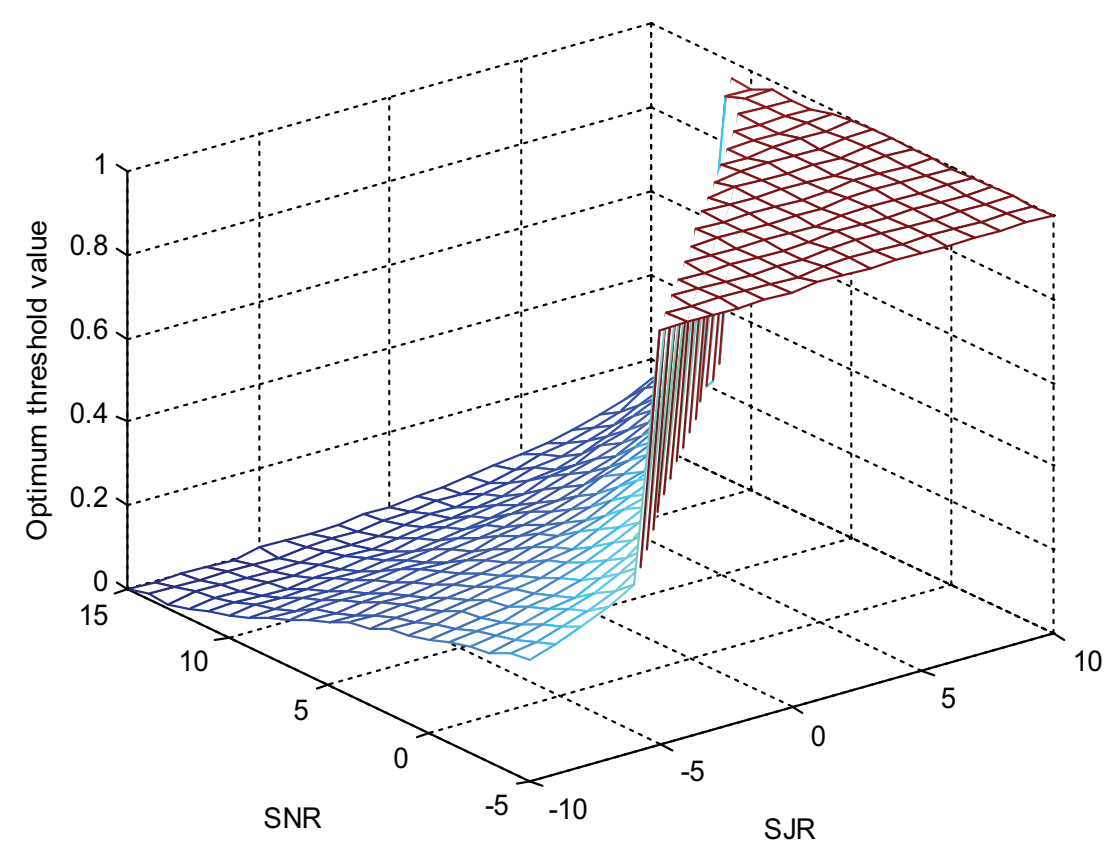

Figure 4.15: The optimum threshold for CWRFH in different jamming power and SNR scenarios.

\subsection{Clipped Weighted Random Frequency Hopping}

Clipped weighted random frequency hopping $(\mathrm{CWRFH})$ is similar to WRFH and AWRFH, but instead uses the clipped channel gains of $\mathrm{CMFH}$,

$$
P_{k}= \begin{cases}\left|H_{k}\right|^{2}-T \max \left|H_{k}\right|^{2} \text { if }\left|H_{k}\right|^{2} \geq T ; \\ 0 & \text { otherwise. }\end{cases}
$$

as the hopping probability distribution (after appropriate normalization). In this case it is necessary to optimize the clipping threshold, $T$, depending on the jamming power and SNR.

The optimum threshold value is shown in Fig. 4.15. Because the optimal value of $T$ is mostly greater than zero, we expect the CWRFH to be no worse than WRFH, which is the same as CWRFH with $T$ fixed at zero. We need to adjust the threshold 


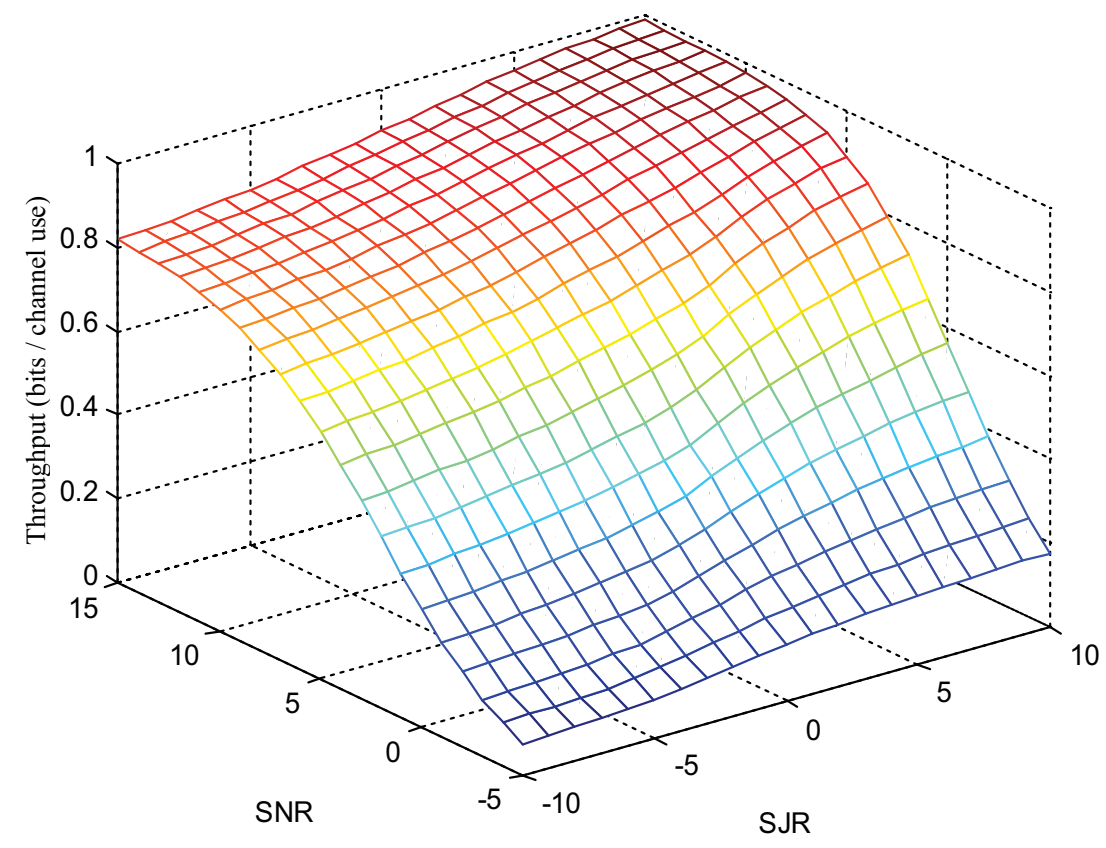

Figure 4.16: The optimum throughput for CWRFH in different jamming power and SNR scenarios.

level to obtain the optimum throughput, which is shown in Fig. 4.16.

We investigated the performance of CWRFH in frequency selective fading with no jamming and we compared it with OCMFH and WRFH. The comparison result is shown in Fig. 4.17. The performance of CWRFH is exactly the same as OCMFH, which is much better performance than WRFH.

The performance in the presence of weak jamming $(\mathrm{SJR}=10 \mathrm{~dB})$ and strong jamming $(\mathrm{SJR}=0 \mathrm{~dB})$ is shown in Fig. 4.18 and Fig. 4.19, respectively. As the jamming power increases the advantage of CWRFH over OCMFH becomes more apparent, although both schemes show a reduced advantage over WRFH.

We also investigated the performance of $\mathrm{CWRFH}$ in the presence of infinite power jamming. The results in Fig 4.20 show that the performance of CWRFH and OCMFH are better than WRFH and the performance of CWRFH is slightly better than OCMFH when the SNR is low and as the SNR increases the performance of 


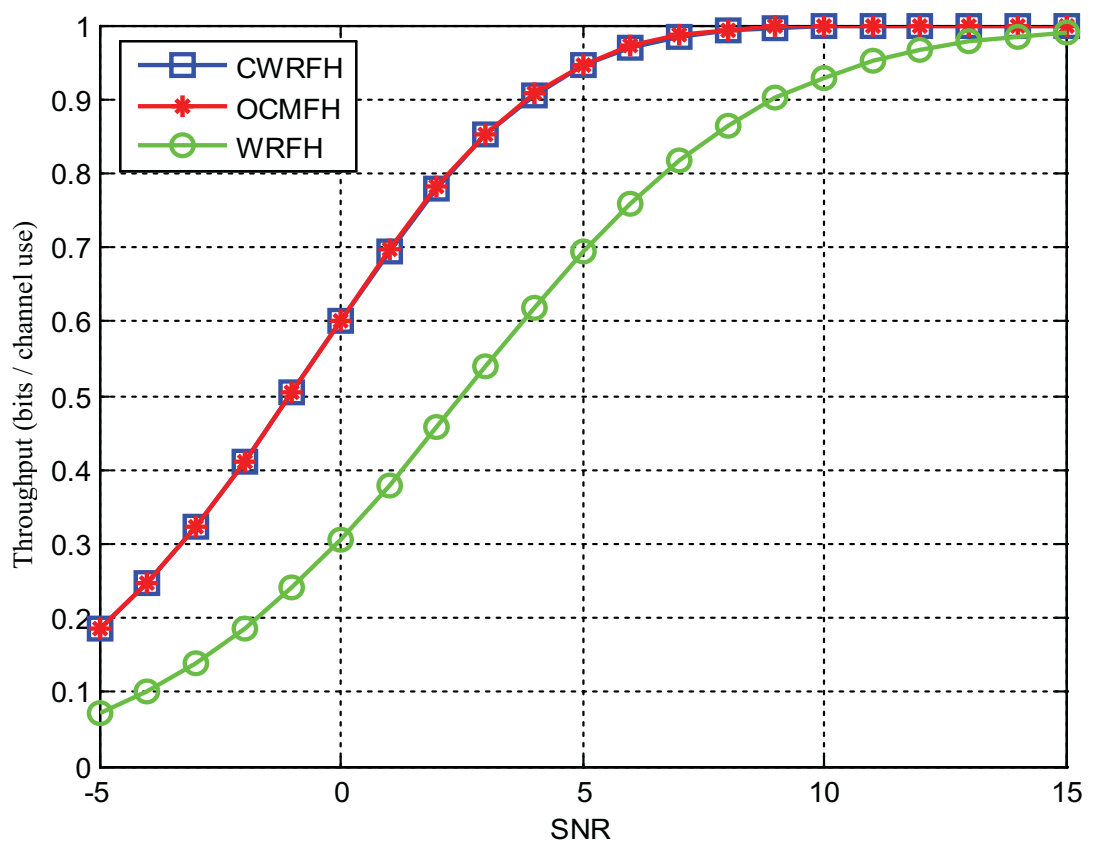

Figure 4.17: The performance of OCWRFH compared to OCMFH and WRFH with absence of jamming.

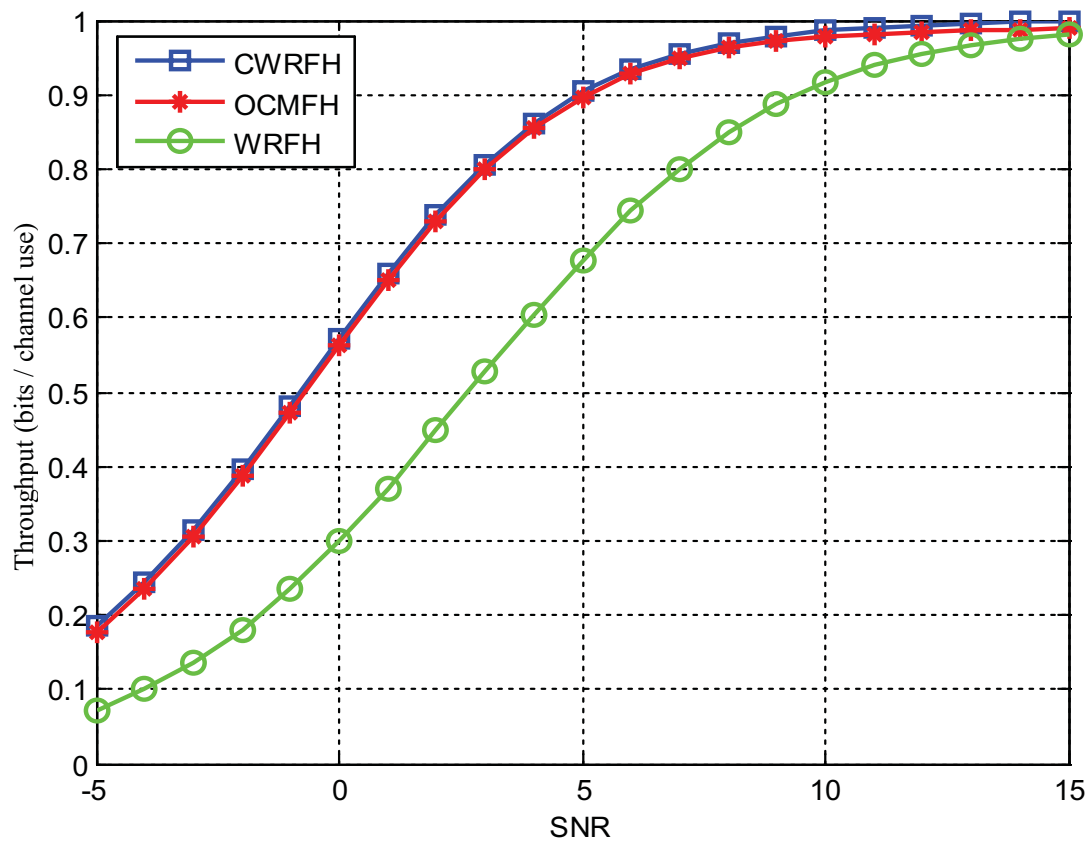

Figure 4.18: The performance of CWRFH compared to OCMFH and WRFH when $\mathrm{SJR}=10 \mathrm{~dB}$. 


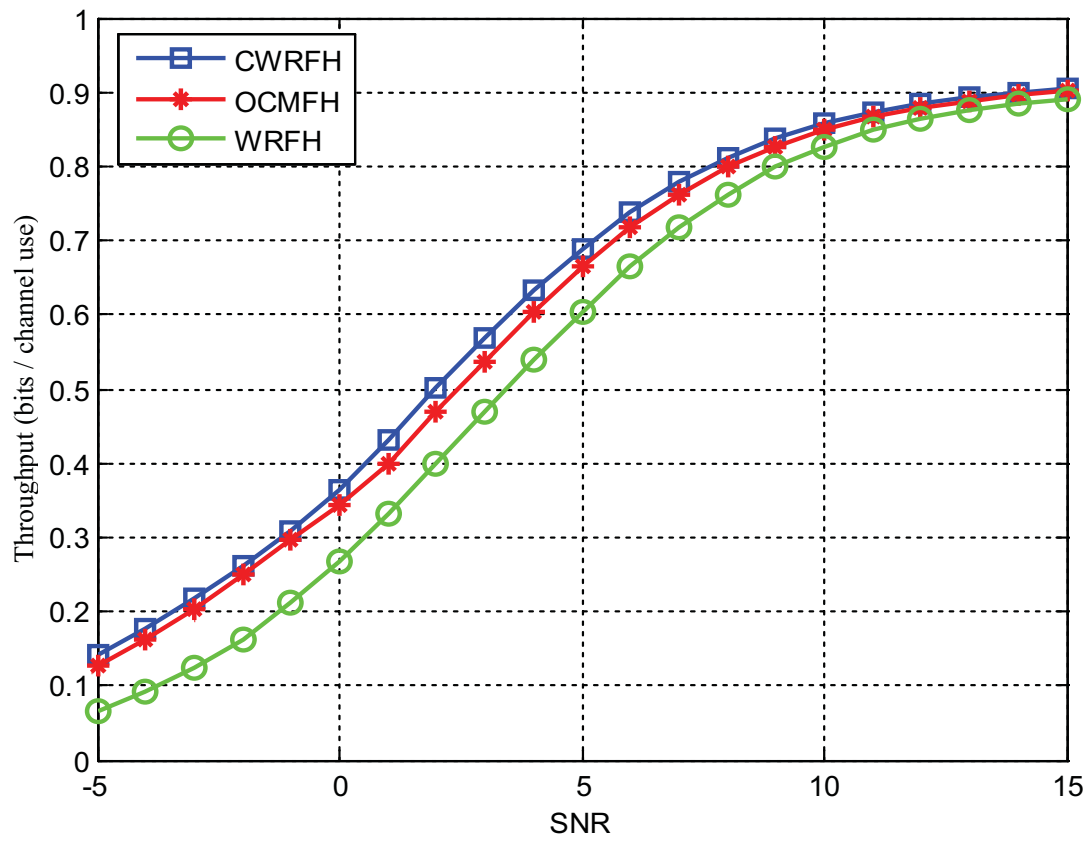

Figure 4.19: The performance of CWRFH compared to OCMFH and WRFH when $\mathrm{SJR}=0 \mathrm{~dB}$.

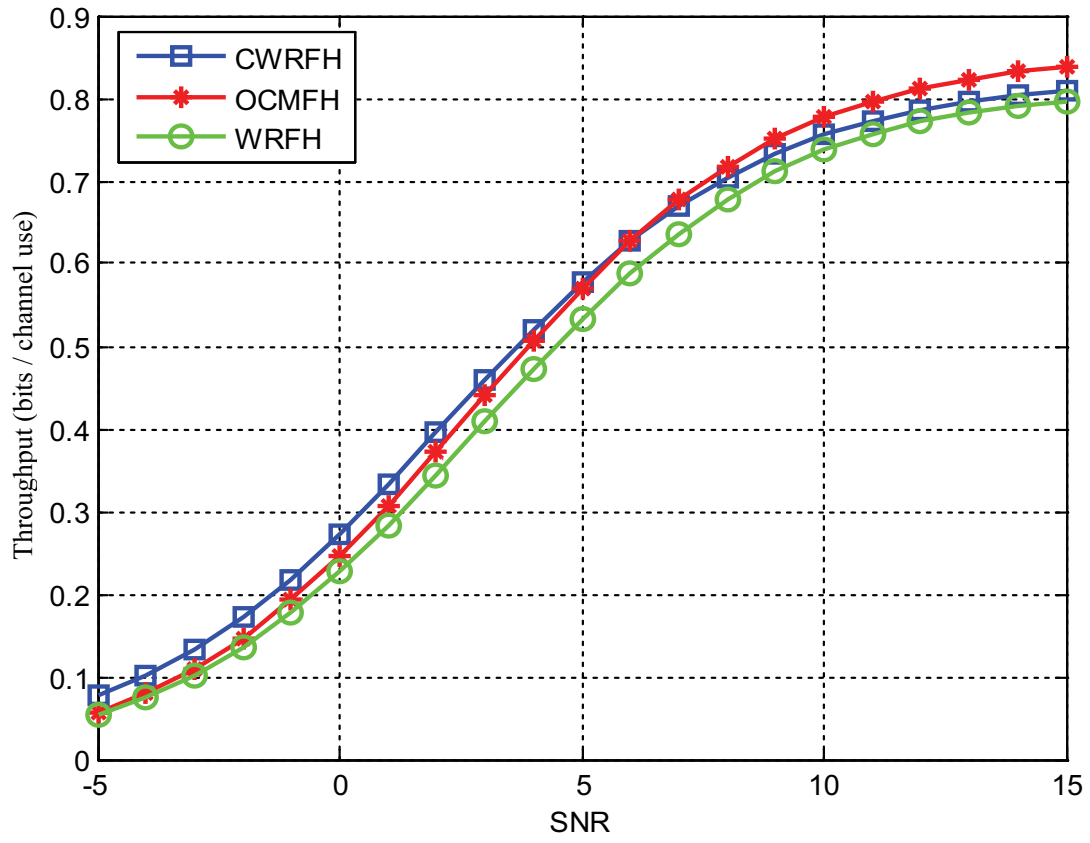

Figure 4.20: The performance of OCWRFH compared to OCMFH and WRFH with the presence of infinite jamming.

OCMFH becomes better than CWRFH. 


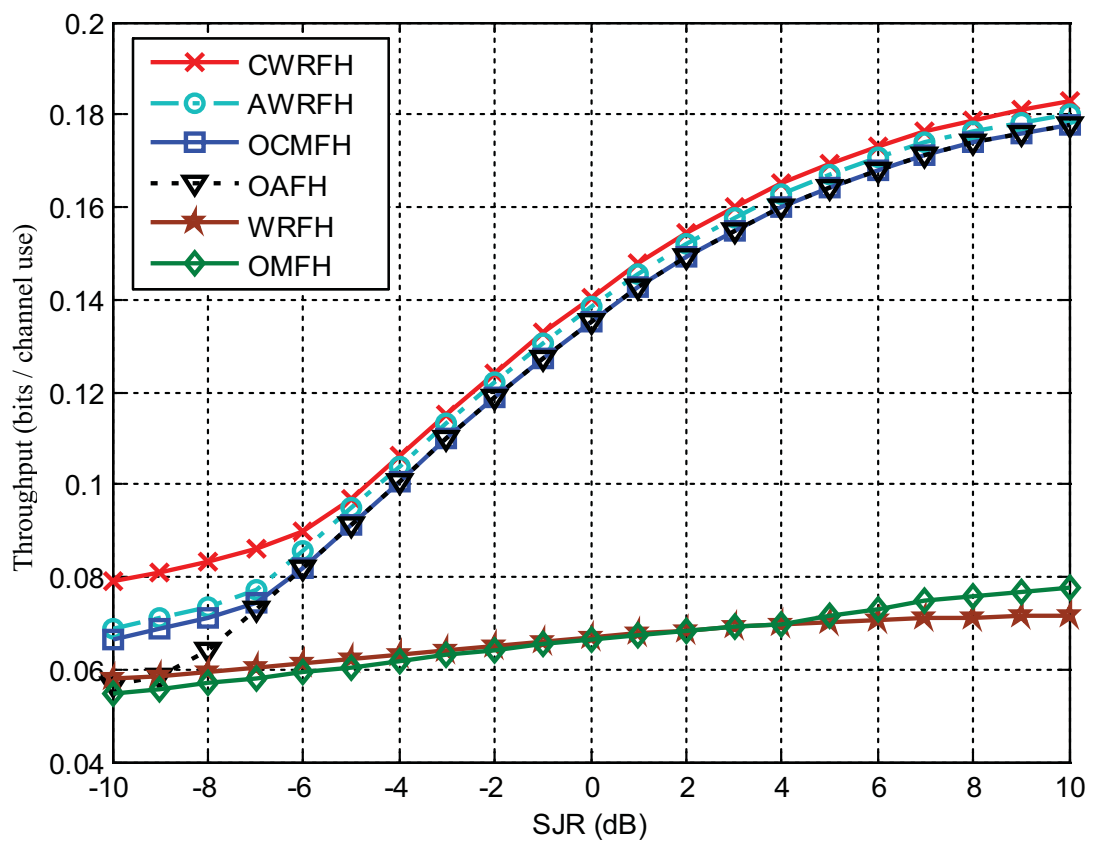

Figure 4.21: The comparison of all proposed schemes vs. SJR when $\mathrm{SNR}=-5 \mathrm{~dB}$.

\subsection{Performance Comparison}

Mitigating jamming in frequency selective fading is the main objective of this thesis and we proposed six effective anti-jamming frequency hopping schemes. In this section we want to compare OMFH, OCMFH, OAFH, WRFH, AWRFH and CWRFH to determine which scheme is better. We compared the performance of these schemes in different jamming power scenarios and SNR values in the WINNER frequency selective fading channel in terms of throughput.

The performance comparison when the $\mathrm{SNR}=-5 \mathrm{~dB}$ is shown in Fig. 4.21. The figure shows that the CWRFH scheme is much better than the other schemes, especially in the presence of strong jamming.

By increasing the SNR to 0,5 and $10 \mathrm{~dB}$ the throughput comparison of all the schemes is shown in Figs. 4.22, 4.23 and 4.24, respectively.

According to this comparison, it is found that the performance of the CWRFH 


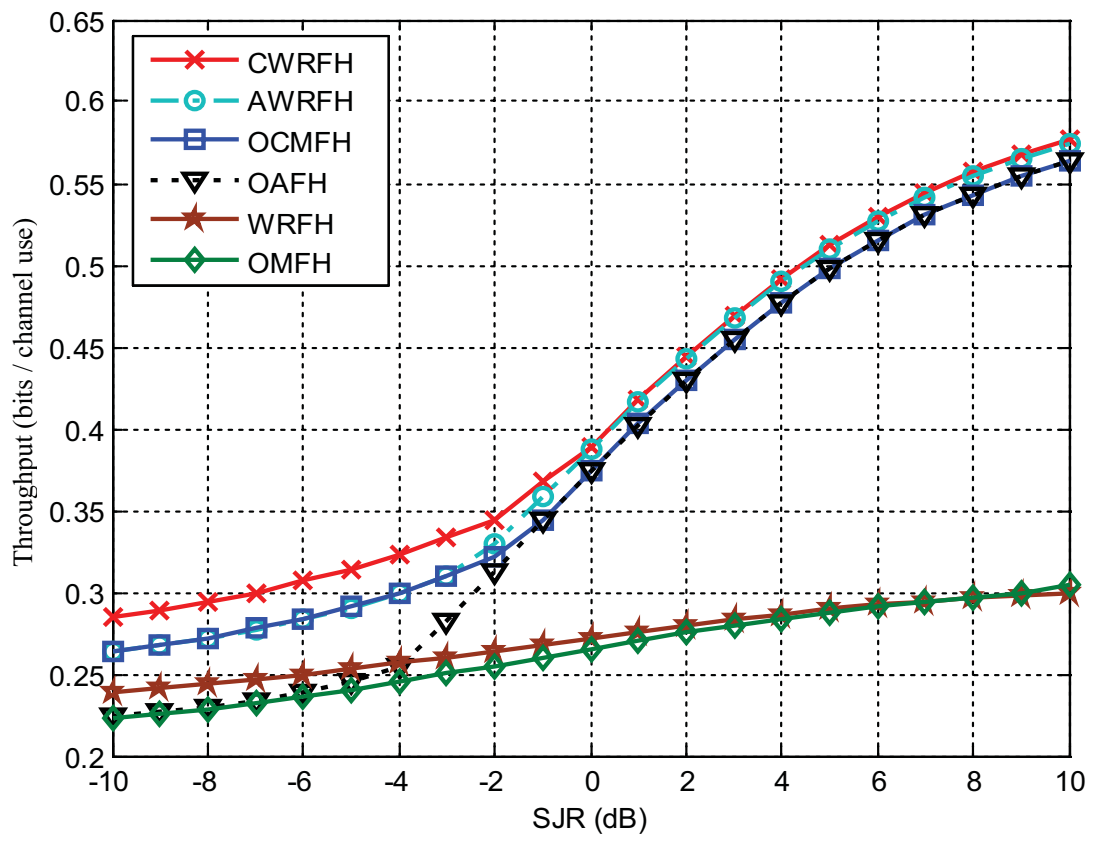

Figure 4.22: The comparison of all proposed schemes vs. SJR when $\mathrm{SNR}=0 \mathrm{~dB}$.

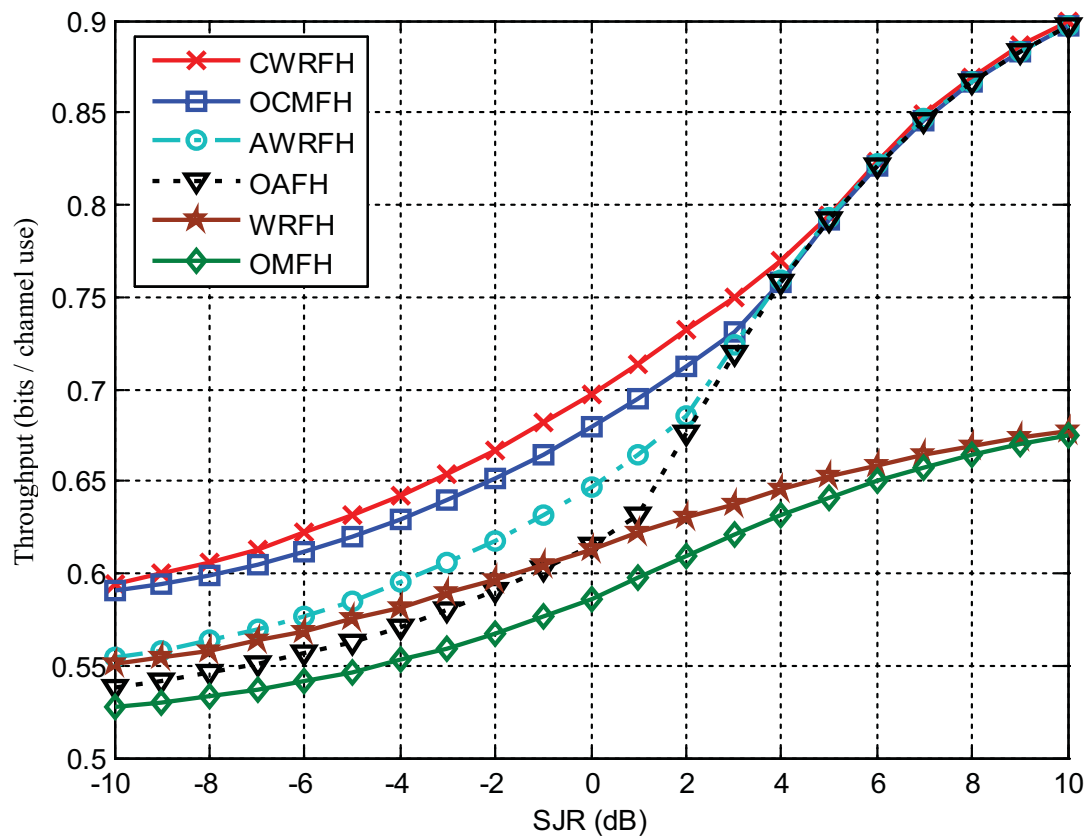

Figure 4.23: The comparison of all proposed schemes vs. SJR when $\mathrm{SNR}=5 \mathrm{~dB}$.

scheme is the best compared to other schemes when the jamming is strong and the SNR value is low. As the jamming power decreases the performance of CWRFH 


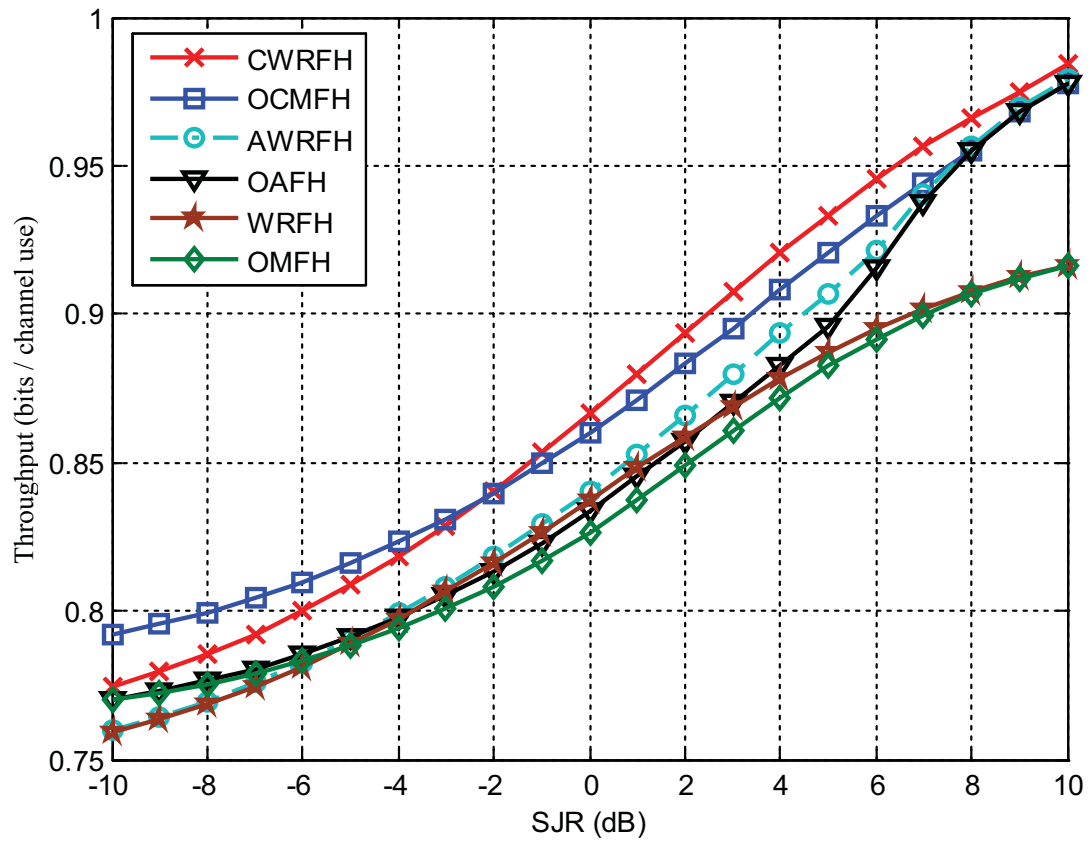

Figure 4.24: The comparison of all proposed schemes vs. SJR when $\mathrm{SNR}=10 \mathrm{~dB}$.

approaches the performance of AWRFH, OCMFH and OAFH schemes. WRFH and OMFH have the worst throughput performance compared to the optimized schemes. CWRFH always gives the best performance, except when the jamming is strong and the SNR is high, in which case OCMFH is better. This is because in the case of CWRFH the jammer can jam grater percentage of the used sub-bands than in case of OCMFH, which assigns the all available sub-bands to the system, and that enhances the throughput performance of the OCMFH especially in high SNR.

\subsection{Conclusion}

In this chapter, a new method of generating frequency hopping patterns is presented. This method, WRFH, has the advantage of selecting the hopping frequencies randomly while avoiding the highly attenuated sub-bands. We were also interested to find new algorithms to mitigate the effect of finite power jamming in frequency 
selective fading. For that reason we proposed two new algorithms: clipped weighted random frequency hopping $(\mathrm{CWRFH})$ and advanced weighted random frequency hopping (AWRFH). We investigated the performance of WRFH, CWRFH and AWRFH in frequency selective fading channel. The proposed CWRFH scheme has the best throughput performance in the absence and in the presence of weak jamming in frequency selective fading. When the SNR becomes very high OCMFH becomes more efficient in the presence of strong jamming. 


\section{Chapter 5}

\section{Conclusions and Future Work}

\subsection{Summary and Conclusions}

Radio signals are received by unwanted listeners, degraded by jammers and corrupted by electromagnetic wave propagation such as fading, shadowing, and multipath propagation. All of these effects result in a performance degradation of wireless communication systems. The main objective of this thesis is to explore different ways to enhance the performance of the systems in the presence of frequency selective fading and jamming.

Frequency hopping is a spread spectrum scheme that is widely used in wireless communication systems. Many channel-aware frequency hopping schemes such as MFH, CMFH and AFH can be used instead of channel-ignorant frequency hopping to enhance the performance of the communication systems. This enhancement is due to the selection of the best available sub-bands in the channel.

Although MFH, CMFH and AFH have previously been proposed, their performance in jamming environments has never been studied. Furthermore, no attempt has been made to optimize their performance by adjusting their parameters $(M, T$ and $\alpha$ ). In Chapter 3 we explored their performance in a realistic adaptive partial band jamming environment and optimized their parameters. We found CMFH to be the best overall scheme when the best values for $T$ and $M$ are chosen based on the 
SNR and jamming power.

To provide even better performance we proposed three new schemes: WRFH, AWRFH and CWRFH. These schemes hop over all the available sub-bands, but hop according to different non-uniform hopping probability distributions. In Chapter 4 we studied the performance of these schemes, and compared them to the optimized versions of MFH, CMFH and AFH. We found that CWRFH provided the best throughput, except in the presence of strong jamming at high SNRs, when OCMFH was the best choice.

\subsection{Suggestions for Future Work}

There are several different ways that this research should be extended in the future. Some of these are as follows:

- We want to find a simple symbolic expression for the threshold value $T$ versus the SNR and SJR for the CWRFH algorithm. This will make the algorithm easier to implement, although it may slightly degrade the performance of the algorithm.

- The feedback requirement for the WRFH-based schemes is higher than for $\mathrm{MFH}, \mathrm{CMFH}$ and $\mathrm{AFH}$. Whereas $\mathrm{MFH}, \mathrm{CMFH}$ and $\mathrm{AFH}$ only need to send a $K$-bits hopping set mask back from the receiver to the transmitter to define the hopping set, the WRFH-based schemes need to send back $K$ probabilities (real numbers) to define the hopping probabilities. It is therefore important to study the impact of quantization of the hopping probabilities to determine the minimum number of quantization levels needed without excessively sacrificing performance. 
- The outage probability performance of all the proposed frequency hopping schemes should be studied in the presence of jamming. The outage probability is the probability that a given rate will not be supported because of channel variation. It is important to consider the outage probability because we are trying to improve the performance in fading. We think that CWRFH will still have the best performance in the presence of jamming because of the optimization that we use to select $T$.

- There are many other choices that could be used for the hopping probabilities for the WRFH-based schemes, and some of these are likely to deliver even better performance. One particularly attractive approach is to use the instantaneous channel capacity given by (2.10) as the hopping probability for each sub-band. 


\section{References}

[1] K. K. Wong and T. O'Farrell, "Spread spectrum techniques for indoor wireless IR communications," IEEE Wireless Communications, vol. 10, no. 2, pp. 54-63, April 2003.

[2] H. Xing and P. Jarske, "Frequency domain equalization for the multicarrier spread spectrum system in multipath fading channel," International Conference on Communication Technology, vol. 2, pp. 603-606, Beijing, China, May 1996.

[3] D. Torrieri, Principles of Spread Spectrum Communication Systems. Springer, 2005.

[4] L. C. Godara, Handbook of Antennas in Wireless Communications. CRC Press, 2002 .

[5] M. G. Sanchez, A. V. Alejos, and I. Cuias, "Indoor radio reception at 60 GHz: Fading mitigation using diversity techniques," IEEE Transactions on Antennas Propagation, vol. 57, no. 10, pp. 2934-2939, October 2009.

[6] O. Alkaf, R. M. Shubair, and K. Mubarak, "Improved performance of MIMO antenna systems for various fading channels," International Conference on Innovations in Information Technology, pp. 13-16, Abu Dhabi, UAE, March 2012.

[7] A. A. Siddiqui, A. U. Khan, M. M. Manzoor, I. A. Awan, A. Ijaz, and S. Chand, "Interference mitigation in 4G system using smart antenna," International Conference on Information and Communication Technologies, Karachi, Pakistan, July 2011.

[8] A. Worthen and W. Stark, "Interference mitigation in frequency hopped spread spectrum systems," Sixth International Symposium on Spread Spectrum Techniques and Applications, vol. 1, pp. 58-62, Parsippany, USA, September 2000.

[9] N. Seshadri, A. R. Calderbank, and G. Pottie, "Channel coding for cochannel interference suppression in wireless communication systems," Vehicular Technology Conference, vol. 2, pp. 629-633, Chicago, USA, July 1995. 
[10] B. Lo and K. Letaief, "Adaptive equalization and interference cancellation for wireless communication systems," IEEE Trans. Commun., vol. 47, no. 4, pp. 538-545, April 1999.

[11] B. B. Pandey and A. Choubey, "Interference mitigation by CDMA rake receiver with walsh-hadamard sequence," International Journal of Advanced Research in Computer Engineering and Technology, vol. 1, no. 5, pp. 60-64, July 2012.

[12] C. Subhas, K. R. Naidu, and Y. V. Reddy, "Pre-rake diversity with generalized orthogonal codes and imperfect channel conditions for FDD/DS-CDMA systems," ARPN Journal of Engineering and Applied Sciences, vol. 4, no. 7, pp. 64-70, September 2009.

[13] N. Damji and T. Le-Ngoc, "Dynamic downlink OFDM resource allocation with interference mitigation and macro diversity for multimedia services in wireless cellular systems," Wireless Communications and Networking Conference, vol. 3, pp. 1298-1304, New Orleans, USA, March 2005.

[14] A. Yahya, O. Sidek, and J. Mohamad-Saleh, "Performance analyses of fast frequency hopping spread spectrum and jamming systems," The International Arab Journal of Information Technology, vol. 5, no. 2, pp. 115-119, April 2008.

[15] F. Hsu and A. Giordano, "Digital whitening techniques for improving spread spectrum communications performance in the presence of narrowband jamming and interference," IEEE Trans. Commun., vol. 26, no. 5, pp. 209-216, February 1978.

[16] A. Viterbi, "Spread spectrum communications-myths and realities," IEEE Communications Magazine, vol. 17, no. 3, pp. 11-18, May 1979.

[17] J. Blanchard, "A slow frequency hopping technique that is robust to repeat jamming," IEEE Military Communications Conference, vol. 1, pp. 14.1-1-14.19, USA, October 1982.

[18] B. Boroujeny and C. Furse, "Robust multicarrier spread spectrum technique for data transmission over partially jammed channels," IEEE Military Communications Conference, vol. 2, pp. 751-757, Monterey, USA, November 2004.

[19] C. Popper, M. Strasser, and S. Capkun, "Anti-jamming broadcast communication using uncoordinated spread spectrum techniques," IEEE Journal on Selected Areas in Communications, vol. 28, no. 5, pp. 703-715, June 2010.

[20] L. Perez-Freire and F. Perez-Gonzalez, "Spread-spectrum watermarking security," IEEE Transactions On Information Forensics ans Security, vol. 4, no. 1, pp. 2-24, March 2009. 
[21] F. Cayre, C. Fontaine, and T. Furon, "Watermarking security: Theory and practice," IEEE Transactions On Signal Processing, vol. 53, no. 10, pp. 39763987, October 2005.

[22] A. M. Alattar, G. I. Al-Regib, and S. A. Al-Semari, "Improved selective encryption techniques for secure transmission of MPEG video bit-streams," International Conference on Image Processing, vol. 4, pp. 256-260, Kobe, Japan, October 1999.

[23] T. Yang and L. O. Chua, "Secure communication via chaotic parameter modulation," IEEE Transactions on Circuits and Systems I: Fundamental Theory and Applications, vol. 43, no. 9, pp. 817-819, September 1996.

[24] R. L. Pickholtz, D. L. Schilling, and L. B. Milstein, "Theory of spread-spectrum communications-A tutorial," IEEE Transaction on Communication, vol. 30, no. 5, pp. 855-884, May 1982.

[25] A. Snider and D. Schilling, "The response of a quasi-SSB-FM system to noise," IEEE Transactions on Communication Technology, vol. 18, no. 6, pp. 763-772, December 1970.

[26] S. P. Lipshitz and J. Vanderkooy, "Pulse-code modulation: An overview," Journal of the AES, vol. 52, no. 3, pp. 200-215, March 2004.

[27] M. K. Simon, J. K. Omura, R. A. Scholtz, and B. K. Levitt, Spread Spectrum Communications Handbook. McGraw-Hill, 2002, vol. 1.

[28] R. Malik, "Spread spectrum - secret military technology to 3G," IEEE Conference on the History of Telecommunications, July 2001.

[29] R. C. Dixon, Spread Spectrum Systems, 2nd ed. Wiley Inter Science, 1984.

[30] S. C. Yang, CDMA RF System Engineering. Artech House, 1998.

[31] J. McCarthy, "A direct sequence spread spectrum overlay for a satellite communication system," in Proc. of the Eighth National Space Engineering Symposium, Brisbane, Australia, September 1993, pp. 227 - 234.

[32] H. Kwon, L. Miller, and J. Lee, "Evaluation of a partial-band jammer with gaussian-shaped spectrum against FH/MFSK," IEEE Transactions on Communication, vol. 38, no. 7, pp. 1045-1049, July 1990.

[33] R. Poisel, Modern Communications Jamming: Principles and Techniques. Artech House, 2004. 
[34] B. McGuffin, "Jammed FH-FSK performance in Rayleigh and Nakagami-m fading," IEEE Military Communications Conference (MILCOM), vol. 2, pp. 1077-1082, Boston, USA, October 2003.

[35] J. Lee, R. French, and L. Miller, "Probability of error analyses of a BFSK frequency-hopping system with diversity under partial-band jamming interference-part I: Performance of square-law linear combining soft decision receiver," IEEE Transactions on Communications, vol. 32, no. 6, pp. 645-653, June 1984 .

[36] B. Levitt, "Use of diversity to improve FH/MFSK performance in worst case partial band noise and multitone jamming," IEEE Military Communications Conference (MILCOM), vol. 2, pp. 28.2-1-28.2-5, USA, October 1982.

[37] M. Simon and A. Polydoros, "Coherent detection of frequency-hopped quadrature modulations in the presence of jamming-part I: QPSK and QASK modulations," IEEE Transactions on Communications, vol. 29, no. 11, pp. 1644-1660, November 1981.

[38] I. Ghareeb and A. Yongacoglu, "Performance analysis of frequency hopped/coherent MPSK in the presence of multitone jamming," IEEE Transactions on Communications, vol. 44, no. 2, pp. 152-155, February 1996.

[39] B. Levitt, "FH/MFSK performance in multitone jamming," IEEE Journal on Selected Areas in Communications, vol. 3, no. 5, pp. 627-643, September 1985.

[40] D. Torrieri, "Fundamental limitations on repeater jamming of frequency-hopping communications," IEEE Journal on Selected Areas in Communications, vol. 7, no. 4, pp. 569-575, May 1989.

[41] X. Meng, R. Tao, and L. Jia, "An intelligent anti-jamming frequency hopping system," First International Conference on Pervasive Computing, Signal Processing and Applications (PCSPA), pp. 1053-1056, Harbin, China, September 2010 .

[42] C. Ko, H. Nguyen-Le, and L. Huang, "Joint interference suppression and symbol detection in slow FH/MFSK systems with an antenna array," IEEE Vehicular Technology Conference (VTC), vol. 6, pp. 2691-2695, Melbourne, Australia, May 2006.

[43] C. Yading, L. Gongming, L. Shaoqian, and C. Yufan, "A new anti-jam receiver for MFSK/FFH system with multitone jamming," 11th IEEE Singapore International Conference on Communication Systems (ICCS), pp. 451-455, Guangzhou, China, November 2008. 
[44] E. Markin, "Jamming detection in providing for radar jamming immunity," IEEE EUROCON Conference, pp. 1565-1567, Saint Petersburg, Russia, May 2009 .

[45] M. Greco, F. Gini, and A. Farina, "Radar detection and classification of jamming signals belonging to a cone class," IEEE Transactions on Signal Processing, vol. 56, no. 5, pp. 1984-1993, February 2008.

[46] H. Nguyen-Le, T. Le-Ngoc, and C. Ko, "Pilot-aided jamming cancelation in slow FH/MFSK systems," International Conference on Advanced Technologies for Communications (ATC), pp. 235-238, Da Nang, Vietnam, August 2011.

[47] Q. Ling, T. Li, and Z. Ding, "A novel concept: Message driven frequency hopping (MDFH)," IEEE International Conference on Communications (ICC), pp. 5496-5501, Glasgow, UK, June 2007.

[48] Q. Ling, J. Ren, and T. Li, "Spectrally efficient spread spectrum system design: Message-driven frequency hopping," IEEE International Conference on Communications (ICC), pp. 4775-4779, Beijing, China, May 2008.

[49] Q. Ling and T. Li, "Message-driven frequency hopping: Design and analysis," IEEE Transactions on Wireless Communications, vol. 8, no. 4, pp. 1773-1782, April 2009.

[50] L. Zhang, H. Wang, and T. Li, "Anti-jamming message-driven frequency hopping-Part I: System design," IEEE Transactions on Wireless Communications, vol. 12, no. 1, pp. 70-79, January 2013.

[51] L. Zhang and T. Li, "Anti-jamming message-driven frequency hopping-Part II: Capacity analysis under disguised jamming," IEEE Transactions on Wireless Communications, vol. 12, no. 1, pp. 80-88, January 2013.

[52] H. Wang, L. Zhang, T. Li, and J. Tugnait, "Spectrally efficient jamming mitigation based on code-controlled frequency hopping," IEEE Transactions on Wireless Communications, vol. 10, no. 3, pp. 728-732, March 2011.

[53] Y. Lam and P. Wittke, "Frequency-hopped spread-spectrum transmission with band-efficient modulations and simplified noncoherent sequence estimation," IEEE Transactions on Communications, vol. 38, no. 12, pp. 2184-2196, December 1990.

[54] R. Robertson and K. Lee, "Performance of fast frequency-hopped MFSK receivers with linear and self-normalization combining in a rician fading channel with partial-band interference," IEEE Journal on Selected Areas in Communications, vol. 10, no. 4, pp. 731-741, May 1992. 
[55] S. Glisic, Z. Nikolic, N. Milosevic, and A. Pouttu, "Advanced frequency hopping modulation for spread spectrum WLAN," IEEE Journal on Selected Areas in Communications, vol. 18, no. 1, pp. 16-29, January 2000.

[56] T. Wu, "A sub-optimal maximum-likelihood receiver for FFH/BFSK systems with multitone jamming over frequency-selective rayleigh fading channels," IEEE Transactions on Vehicular Technology, vol. 57, no. 2, pp. 1316-1322, March 2008.

[57] D. Torrieri, S. Cheng, and M. Valenti, "Robust frequency hopping for interference and fading channels," IEEE Transactions on Communications, vol. 56, no. 8, pp. 1343-1351, August 2008.

[58] K. Hamdi and O. Bamahdi, "A new adaptive frequency hopping technique," IEEE Vehicular Technology Conference (VTC), vol. 3, pp. 2083-2086, Los Angeles, USA, September 2004.

[59] Z. Kostic, I. Maric, and X. Wang, "Fundamentals of dynamic frequency hopping in cellular systems," IEEE Journal on Selected Areas in Communications, vol. 19, no. 11, pp. 2254-2266, November 2001.

[60] O. Mubarek, H. Yanikomeroglu, and S. Periyalwar, "Dynamic frequency hopping in cellular fixed relay networks," IEEE Vehicular Technology Conference (VTC), vol. 5, pp. 3112-3116, Stockholm, Sweden, May 2005.

[61] S. Tomisato, K. Fukawa, and H. Suzuki, "Coherent frequency hopping multiple access (CFHMA) with multiuser detection for mobile communication systems," IEEE Transactions on Vehicular Technology, vol. 49, no. 2, pp. 531-539, March 2000 .

[62] R. Kozick and B. Sadler, "Maximum likelihood multi-user detection for fast frequency hopping/multiple frequency shift keying systems," Wireless Communications and Networking Conference (WCNC), vol. 1, pp. 67-72, Chicago, USA, September 2000.

[63] A. Correia and A. Rodrigues, "Adaptive frequency-hopping for TDMA/CDMA," IEEE Vehicular Technology Conference (VTC), vol. 3, pp. 2212-2216, Ottawa, Canada, May 1998.

[64] M. Elkashlan and C. Leung, "A channel aware frequency hopping multiple access scheme," IEEE International Conference on Communications, vol. 1, pp. 390394, Paris, France, June 2004. 
[65] M. Elkashlan, C. Leung, and R. Schober, "Performance analysis of channel aware frequency hopping," Proc. IEEE Communications, vol. 153, no. 6, pp. 841-845, December 2006.

[66] T. Jia and A. Duel-Hallen, "Subchannel allocation for multicarrier CDMA with adaptive frequency hopping and decorrelating detection," IEEE Military Communications Conference (MILCOM), pp. 1-7, Washington D.C., USA, October 2006 .

[67] P. Popovski, H. Yomo, and R. Prasad, "Strategies for adaptive frequency hopping in the unlicensed bands," IEEE Wireless Communications, vol. 13, no. 6, pp. 60-67, December 2006.

[68] P. Popovski, H. Yomo, and R. Prsad, "Dynamic adaptive frequency hopping for mutually interfering wireless personal area networks," IEEE Transactions on Mobile Computing, vol. 5, no. 8, pp. 991-1003, August 2006.

[69] S. Lee and Y. Lee, "Adaptive frequency hopping for bluetooth robust to WLAN interference," IEEE Communications Letters, vol. 13, no. 9, pp. 628630, September 2009.

[70] Y. Wenlong, C. Yunpeng, and S. Liang, "Adaptive frequency-hopping in HF communications," International Conference on Transportation, Mechanical, and Electrical Engineering, pp. 427-430, Changchun, China, December 2011.

[71] S. E. El-khamy, "Matched frequency-hopping (MFH) signals for slowly fading dispersive channel," IEEE Transactions on Vehicular Technology, vol. 47, no. 1, February 1998.

[72] S. E. El-khamy, F. Abdalla, and A. Al-Hourani, "An improved channel aware clipped matched frequency hopping $(\mathrm{CMFH})$ signaling technique in slowly fading dispersive channels," Twenty-Second National Radio Science Conference,, vol. 36, pp. 506-509, Cairo, Egypt, April 2005.

[73] S. E. El-khamy and W. M. Saad, "New technique for enhancing the signaling in slowly fading dispersive channels," International Conference on Wireless Communications, Networking and Mobile Computing (WiCom), vol. 2, pp. 1-4, Beijing, China, September 2009.

[74] A. Shokrollahi, "Raptor codes," IEEE Transactions on Information Theory, vol. 52, pp. 2551-2567, June 2006. 
[75] J. J. Perez-Solano, S. Felici-Castell, and M. A. Rodriguez-Hernandez, "Narrowband interference suppression in frequency-hopping spread spectrum using undecimated wavelet packet transform," IEEE Transactions On Vehicular Technology, vol. 57, no. 3, pp. 1620-1629, May 2008.

[76] X. Liu, N. Sidiropoulos, and A. Swami, "Blind high-resolution localization and tracking of multiple frequency hopped signals," IEEE Transactions on Signal Processing, vol. 50, no. 4, pp. 889-901, April 2002.

[77] W. M. Saad and I. Marsland, "Jamming and fading channels mitigation using anti-jamming advanced frequency hopping," International Conference on Electrical and Computer Systems, Ottawa, Canada, August 2012.

[78] W. Mohr, "The WINNER (wireless world initiative new radio) project development of a radio interface for systems beyond 3G," International Journal of Wireless Information Networks., vol. 14, no. 2, pp. 67-78, June 2007.

[79] M. Salem, A. Adinoyi, M. Rahman, H. Yanikomeroglu, D. Falconer, Y. Kim, E. Kim, and Y. Cheong, "An overview of radio resource management in relayenhanced OFDMA-based networks," IEEE Communications Surveys and Tutorials, pp. 422-438, Third Quarter 2010.

[80] S. A. Butman, I. Bar-david, B. K. Levitt, R. F. Lyon, and M. J. Klass, "Design criteria for noncoherent gaussian channels with MFSK signaling and coding," IEEE Trans. Commun., vol. 24, no. 10, pp. 1078-1088, October 1976. 


\section{Appendix A}

\section{Throughput in Wide Band Jamming}

In noncoherent MFSK systems the received signal in the presence of additive white Gaussian noise $w(t)$, and in the presence of jamming $w_{J}(t)$ is given by

$$
r(t)=\sqrt{2 S} x_{m}(t) \cos (w t+\theta)+w(t)+w_{J}(t) .
$$

where $S$ is the signal power, $x_{m}(t)$ is one of the $M=2^{K}$ hopping signals, $m=$ $1,2,3, \ldots, M$, that modulated onto a carrier $\cos (w t), \theta$ is the uniformly distributed phase shift.

Using optimum receiver:-

$$
r_{k}=\frac{\sqrt{\frac{2}{T}}}{\sigma}\left[\left(\int_{0}^{T} r(t) x_{k}(t) \cos (w t) d t\right)^{2}+\left(\int_{0}^{T} r(t) x_{k}(t) \sin (w t) d t\right)^{2}\right]^{\frac{1}{2}}
$$

In the presence of wide band jamming the noise level will be increased and $\sigma$ will be given as

$$
\sigma=\sqrt{\frac{E_{J}}{B W}+\frac{N_{0}}{2}}=\sqrt{\frac{2 * E_{J}+N_{0} * B W}{2 * B W}}
$$

where $T$ is the signal time interval, $\frac{E_{J}}{B W}$ is the power spectral density of the jammer, $B W$ is the jammer bandwidth, $E_{J}$ is jammer power and $N_{0}$ is the singlesided white Gaussian noise spectral density. In the absence of jamming $\sigma=\sqrt{\frac{N_{0}}{2}}$. 
By substituting with $\sigma$ from A.3 into A.2 we get

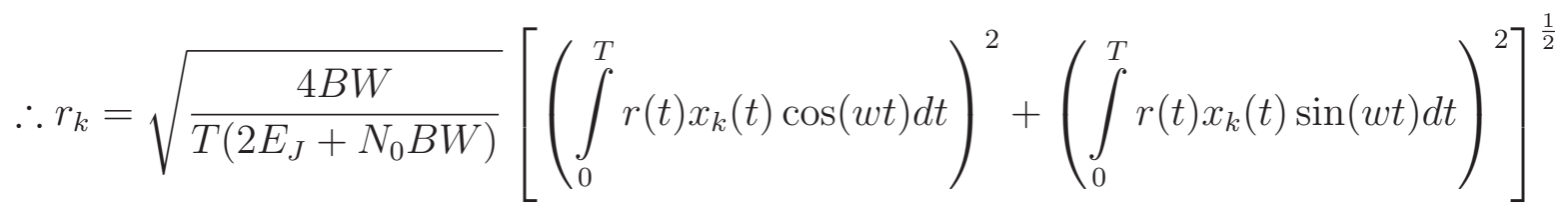

The power density function (pdf) of $r_{k}$ conditioned on transmitting the $m^{\text {th }}$ signal will be as follow

$$
p\left(r_{k} / m\right)= \begin{cases}r_{k} e^{-\frac{1}{2} r_{k}^{2}} & \text { if } k \neq m ; \\ r_{m} e^{-\frac{1}{2} r_{m}^{2}-\frac{1}{2} \alpha^{2}} I_{0}\left(\alpha r_{m}\right) & \text { if } k=m .\end{cases}
$$

$r_{k}$ 's are statically independent random variables.

$$
\rho=\frac{\alpha^{2}}{2}=\frac{S T}{2\left(\frac{E_{J}}{B W}+\frac{N_{0}}{2}\right)}=\frac{S T}{\frac{2 E_{J}}{B W}+N_{0}}
$$

where $\rho$ is the signal-to-jamming and noise ratio (SJNR), $S$ is the transmitted signal power and $T$ is the signal time duration.

$$
I_{0}(\alpha r)=\frac{1}{2 \pi} \int_{-\pi}^{\pi} e^{\alpha r \cos (x)} d x
$$

The conditional probability density of the M-vector $r$ can be calculated from A.5 and it is given by

$$
p(r / m)=g(r) e^{-\frac{1}{2} \alpha^{2}} \times I_{0}\left(\alpha r_{m}\right)
$$

where

$$
g(r)=\prod_{k=1}^{M} r_{k} e^{-\frac{1}{2} r_{k}^{2}}
$$


The information content is

$$
I_{M}(\alpha, P)=\int_{r} \sum_{m=1}^{M} P_{m} p(r / m) \times \ln \left[\frac{p(r / m)}{\sum_{j=1}^{M} P_{j} p(r / j)}\right] d r
$$

where $P=P_{1}, P_{2}, \ldots \ldots \ldots . ., P_{M}$ is the vector of probabilities of the input signals $\left(x_{1}, x_{2}, \ldots \ldots \ldots, x_{M}\right)$.

$$
\begin{aligned}
I_{M}(\alpha, P) & =\int \sum_{m=1}^{M} P_{m} \times g(r) \times e^{-\frac{1}{2} \alpha^{2}} \times I_{0}\left(\alpha r_{m}\right) \times \ln \left[\frac{g(r) e^{-\frac{1}{2} \alpha^{2}} \times I_{0}\left(\alpha r_{m}\right)}{g(r) e^{-\frac{1}{2} \alpha^{2}} \times \sum_{k=1}^{M} P_{k} I_{0}\left(\alpha r_{k}\right)}\right] d r \\
& =\int \sum_{m=1}^{M} P_{m} \times g(r) \times e^{-\frac{1}{2} \alpha^{2}} \times I_{0}\left(\alpha r_{m}\right) \times \ln \left[\frac{I_{0}\left(\alpha r_{m}\right)}{\sum_{k=1}^{M} P_{k} I_{0}\left(\alpha r_{k}\right)}\right] d r \\
& =e^{-\frac{1}{2} \alpha^{2}} \int g(r) \sum_{m=1}^{M} P_{m} \times I_{0}\left(\alpha r_{m}\right) \times \ln \left[\frac{I_{0}\left(\alpha r_{m}\right)}{\sum_{k=1}^{M} P_{k} I_{0}\left(\alpha r_{k}\right)}\right] d r \\
& =e^{-\frac{1}{2} \alpha^{2}} E\left[\sum_{m=1}^{M} P_{m} \times I_{0}\left(\alpha r_{m}\right) \ln \left[I_{0}\left(\alpha r_{m}\right)\right]\right] \\
& -e^{-\frac{1}{2} \alpha^{2}} E\left[\sum_{m=1}^{M} P_{m} \times I_{0}\left(\alpha r_{m}\right) \ln \left[\sum_{m=1}^{M} P_{k} I_{0}\left(\alpha r_{k}\right)\right]\right]
\end{aligned}
$$

Maximizing $I_{M}(\alpha)$ over $P$ by choosing $P_{1}=P_{2}=\ldots \ldots \ldots . .=P_{M}=\frac{1}{M}$

$$
\begin{gathered}
\therefore \sum_{m=1}^{M} P_{m}=\sum_{m=1}^{M} \frac{1}{M}=1 \\
\therefore I_{M}(\alpha)=e^{-\frac{\alpha^{2}}{2}}\left(E\left[I_{0}\left(\alpha r_{1}\right) \times \ln \left[I_{0}\left(\alpha r_{1}\right)\right]\right]-E\left[\frac{S_{M}}{M} \ln \left(\frac{S_{M}}{M}\right)\right]\right)
\end{gathered}
$$


where $S_{M}=\sum_{m=1}^{M} I_{0}\left(\alpha r_{m}\right)$.

For $M=2$, we find that $S_{2}=I_{0}\left(\alpha r_{1}\right)+I_{0}\left(\alpha r_{2}\right)$.

$$
\begin{aligned}
\therefore I(\alpha) & =e^{-\frac{\alpha^{2}}{2}}\left(E\left[I_{0}\left(\alpha r_{1}\right) \times \ln \left[I_{0}\left(\alpha r_{1}\right)\right]\right]-E\left[\frac{1}{2}\left(I_{0}\left(\alpha r_{1}\right)+I_{0}\left(\alpha r_{2}\right)\right) \ln \left(\frac{I_{0}\left(\alpha r_{1}\right)+I_{0}\left(\alpha r_{2}\right)}{2}\right)\right]\right) \\
& =e^{-\frac{\alpha^{2}}{2}} \times \int_{0}^{\infty} I_{0}\left(\alpha r_{1}\right) \times \ln \left[I_{0}\left(\alpha r_{1}\right)\right] p\left(r_{1}\right) d r_{1}-e^{-\frac{\alpha^{2}}{2}} \times \int_{0}^{\infty} \int_{0}^{\infty} \frac{I_{0}\left(\alpha r_{1}\right)+I_{0}\left(\alpha r_{2}\right)}{2} \\
& \times \ln \left(\frac{I_{0}\left(\alpha r_{1}\right)+I_{0}\left(\alpha r_{2}\right)}{2}\right) p\left(r_{1}\right) p\left(r_{2}\right) d r_{1} d r_{2} \\
& =e^{-\frac{\alpha^{2}}{2}} \times \int_{0}^{\infty} I_{0}\left(\alpha r_{1}\right) \times \ln \left(I_{0}\left(\alpha r_{1}\right)\right) \times r_{1} e^{-\frac{1}{2} r_{1}^{2}} d r_{1}-e^{-\frac{\alpha^{2}}{2}} \times \int_{0}^{\infty} \frac{I_{0}\left(\alpha r_{1}\right)+I_{0}\left(\alpha r_{2}\right)}{2} \\
& \times \ln \left(\frac{I_{0}\left(\alpha r_{1}\right)+I_{0}\left(\alpha r_{2}\right)}{2}\right) \times r_{1} e^{-\frac{1}{2} r_{1}^{2}} \times r_{2} e^{-\frac{1}{2} r_{2}^{2}} d r_{1} d r_{2} \\
& =\int_{0}^{\infty} I_{0}\left(\alpha r_{1}\right) \times \ln \left(I_{0}\left(\alpha r_{1}\right)\right) \times r_{1} e^{-\frac{r_{1}^{2}+\alpha^{2}}{2}} d r_{1}-\int_{0}^{\infty} \int_{0}^{\infty} \frac{1}{2}\left[I_{0}\left(\alpha r_{1}\right)+I_{0}\left(\alpha r_{2}\right)\right] \\
& \times r_{1} r_{2} \times \ln \left(\frac{1}{2}\left[I_{0}\left(\alpha r_{1}\right)+I_{0}\left(\alpha r_{2}\right)\right]\right) e^{-\frac{r_{1}^{2}+r_{2}^{2}+\alpha^{2}}{2}} d r_{1} d r_{2}
\end{aligned}
$$

